*ak RMIS View/Frint Document Cover Sheet tow

This document was retrieved from the Documentation and Records Manaqement (DRM) ISEARCH System. It is intended for Information only and may not be the most recent or updated version. Contact a Document Service Center (see Hanford Info for locations) if you need additional retrieval information.

Accession \#: D196001239

Document \#: SD-SNF-DP-003

Title/Desc:

DATA COMPILATION REPORTFOR K WEST BASIN FUEL STORAGE CANISTER LIQUID SAMPLES

Pages: 133 


\begin{tabular}{|c|c|}
\hline $\begin{array}{l}\text { 2. To: (Receiving orgmization) } \\
\text { DISTRIBUTION }\end{array}$ & $\begin{array}{l}\text { 3. Frow: coriginat } \\
8 M 710\end{array}$ \\
\hline $\begin{array}{l}\text { 5. Proj./Pros./Pcpt./DIV.: } \\
\text { SNFP/FUEL CHARACTERIZ }\end{array}$ & $\begin{array}{l}\text { 6. cog. Engr.: } \\
\text { D. J. TRIMBLE }\end{array}$ \\
\hline
\end{tabular}

8. Orlainator Remarke:

DISTRIBUTION OF KH BASIN CANISTER SAMPLE DATA

11. Receiver Remorks:

NA

4. Related EDT No.:

MA

7. Purchase Order Mo.:

NA

9. Equip./Component No.:

NA

10. Syatca/bldo./Facil ity: $105 \mathrm{~K}$ WEST BASIN

12. Mojor Aasm. Dwo. Mo.: NA

13. Pennit/Pernit Application No.: NA

14. Recultred Reepense Dates

NA

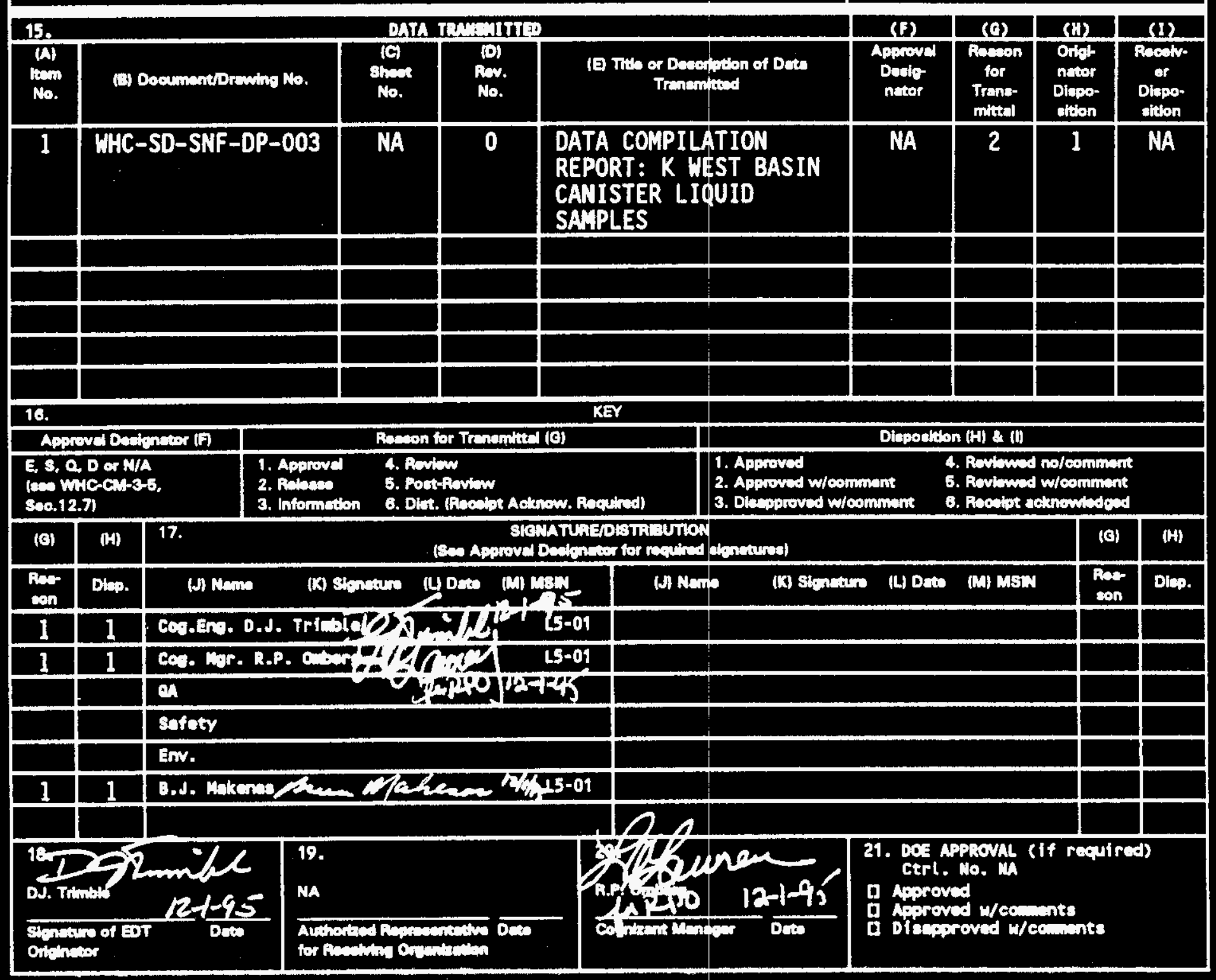




\title{
DATA COMPILATION REPORT: K WEST BASIN FUEL STORAGE CANISTER LIQUID SAMPLES
}

\author{
D. J. Trimble \\ Westinghouse Hanford Co., Richland, WA 99352 \\ U.S. Department of Energy Contract DE-ACOS-87RL10930 \\ EDT/ECN: 610186 \\ UC: 2070 \\ Org Code: 8n710 \\ BAR Code: EN3135040 \\ Charge Code: LE022 \\ Totai Pages: 129
}

Key Words: 105-K West Basin, Fuel Characterization, Spent Nuclear Fuel, N-Reactor Fue1, Liquid Samples, SNFP, Canister Sampi ing, 222-S Laboratory

Abstract: Sample analysis data from the 222-S Laboratory are reported. The data are for liquid samples taken from spent fuel storage canisters in the $105 \mathrm{~K}$ West Bas in during March 1995. An analys is and data report from the Special Analytical Studies group of Westinghouse Hanford Company regarding these samples is also included. Data analys is is not included herein.

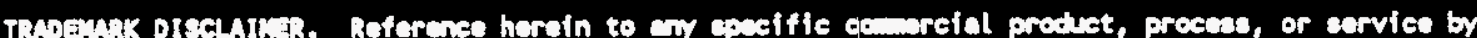

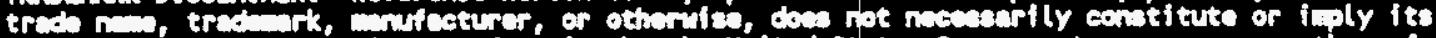

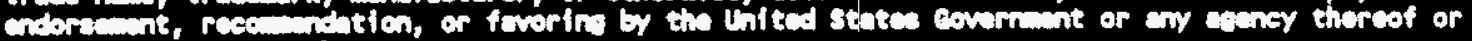
Its centractore or arecintruters.

Printed in the United states of Amprien. To chtain ecpics of this doenent, centect: wuc/pes

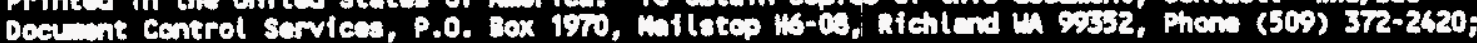
Fax (509) 376-4909.

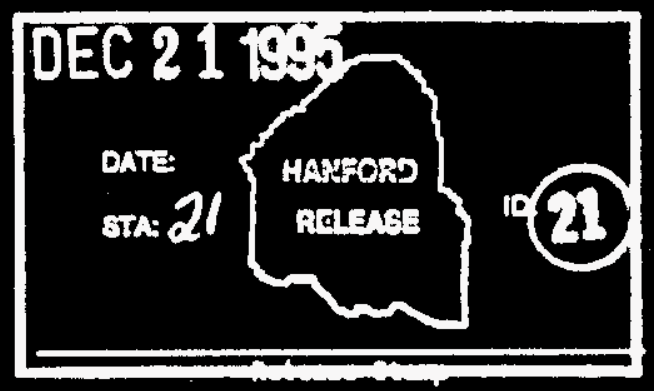


The following trademarks were used in Appendix A of this report.

'NOMAD-Plus is a trademark of the EG\&G ORTEC Company, Oakridge, TN.

${ }^{2}$ MAESTRO is a trademark of the EG\&G ORTEC Company, Oakridge, TN.

${ }^{3} \mathrm{OSQ}^{+}$is a trademark of APTEC Nuclear Inc, Lewiston, NY.

"EXCEL is a trademark of Microsoft Corporation, Redmond, WA.

5WINBATCH is a trademark of Wilson WindowWare, Inc., Seattle, WA.

${ }^{6}$ PopTop is a trademark of the EG\&G ORTEC Company, Oakridge, TN.

Microshield is a trademark of Grove Engineering, Inc., Rockville, MD.

${ }^{8}$ Visual Basic is a Trademark of Microsoft Corporation, Redmond, WA. 


\section{CONTENS}

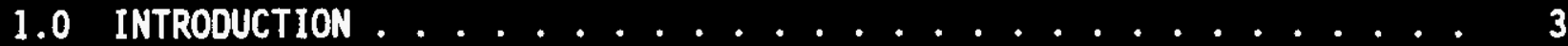

2.0 SAMPLE ANALYSIS DATA ................. 3

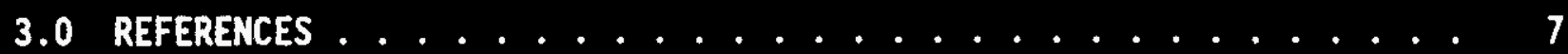

APPENDIX A LETTER, E. F. RIEDEL TO D. J. TRIMBLE,

"RADIONUCLIDE CHARACTERIZATION OF 105-K WEST SPENT FUEL

STORAGE CANISTER SAMPLES," SPECIAL AMALYTICAL STUDIES,

JULY 17, 1995, OWH31-95-0717-1, WEST INGHOUSE HANFORD

COMPANY, RICHLAND, MASHINGTON .............8 8

APPENDIX B PAGES I THROUGH IV AND 1 THROUGH 53

OF WHC-SD-WW-SP-138, REV. 0, "ANALYSIS OF K WEST

BASIN CANISTER LIQUID SAMPLES," G. L. MILLER, 1995,

WESTINGHOUSE HANFORD CONPANY, RICHLAND, WASHINGTON . . . . . 70 
WHC-SD-SNF-DP-003, Rev. 0

DATA COMPILATION REPORT: $K$ WEST BASTM FUEL STORAGE CANISTER LIQUID SAMPLES

\subsection{INRRODUCTION}

Liquid and gas samples were taken from ten fuel storage canisters in the $K$ West Bas in in March 1995. The samples were taken in support of the fuel characterization program and in accordance with the sampling and analys is pian (Harris 1995, SAP). The samples were analyzed for radionuclides, transuranic elements, corroston inhibitor chemicals, and gas species. Samples analyses were performed in a mobile laboratory setting at the $K$ West Basin as well as at the 222-S Laboratory and the 325 Building Laboratory. A description of the sampling process was previously reported with analysis data from the mobile laboratory and the 325 Building (Trimble 1995a). This report documents sample analysis descriptions and data from the 222-S Laboratory. A report of the mobtle laboratory analyses by the Westinghouse Hanford Company Special Analytical Studies (SAS) group is also included.

A partial analysis of the gas and liquid data has been documented (Trimble 1995b). A more complete analysis including the 222-S Laboratory data will follow.

\subsection{SIMLE NMLYSIS DATA}

A report of the mobile laboratory analysis methods and data by SAS (Riede1 1995) is attached in Appendix A. A report of the 222-S Laboratory analys is methods and data has been issued (Miller 1995). The narrative and sample data summary portions of this report are included in Appendix B.

Summaries of the 222-S Laboratory analysis data are provided in Tables 1 and 2. Canister 0000 was an empty canister containing only deionized water from which "blank" sanples were obtained.

A summary of the mobile laboratory and gas analysis data previously reported (Trimble 1995a) is provided for reference (Table 3). Table 3 does not include data from obviously bad samples.

The net weights of the samples were used to calculate Cs-137 activity per gram of sample in Table 3 . In (Trimble 1995a), the specific activity was calculated per gran of net sample weight (KSW). KSW was defined as net weight minus 1 gram, to account for non-canister water taken into the sample. 


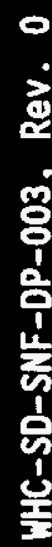

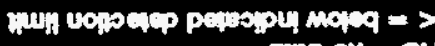
ciep of $=$ on

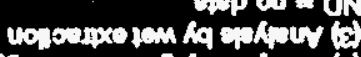

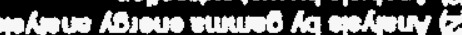

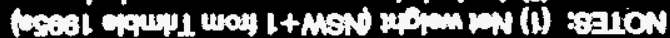

它

응

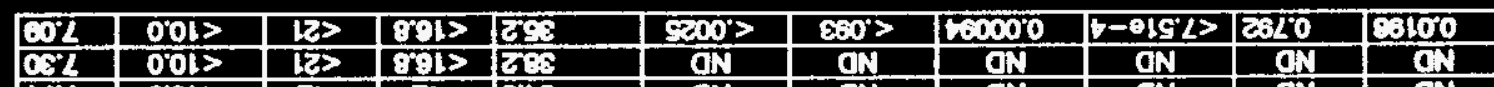

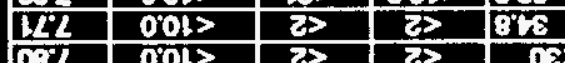

C:2

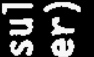

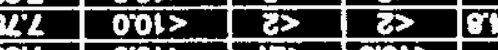
8

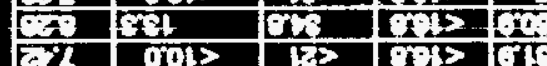

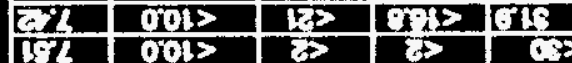

+ै 용

送

क量

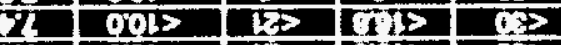

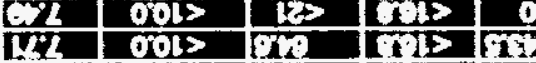

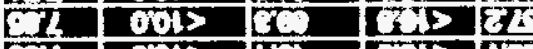

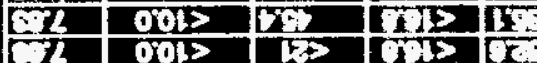

$\underset{2}{0}$ 2 Gi 13

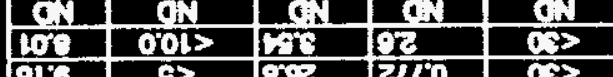

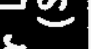

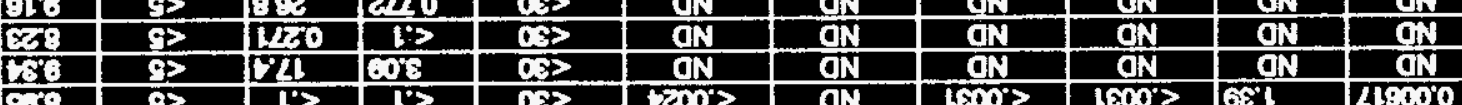

606

$\frac{\cos 20}{100}$

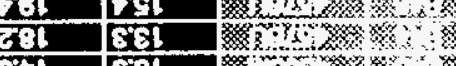

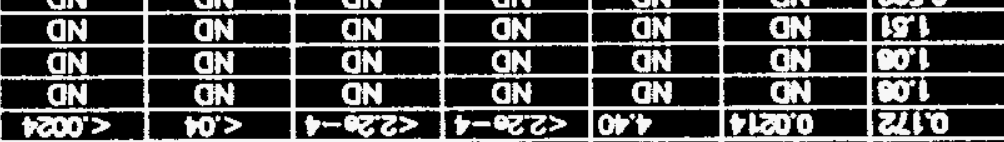
tows Ort 10 t कि -

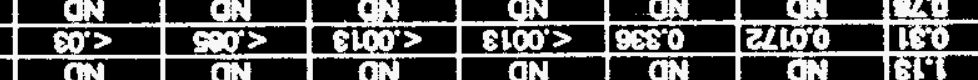
(a) Fin Th

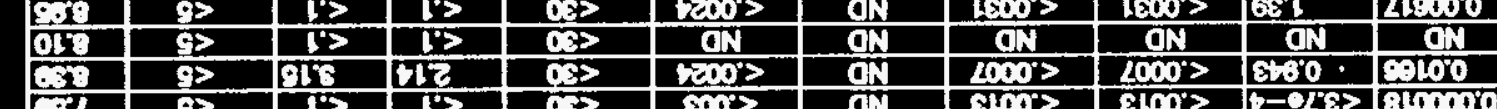

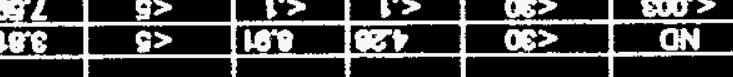

(I) ON

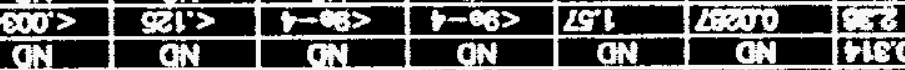

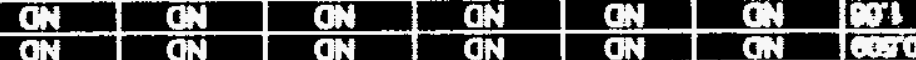
ins

(i)

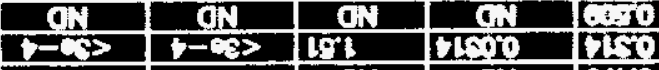

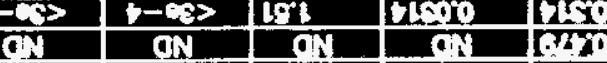
WI 01 Dor \%x (r) as Sol ar al

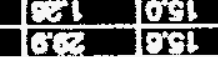
(1) ind

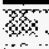
Oij

कO

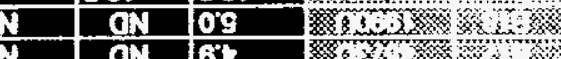

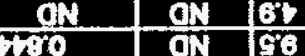

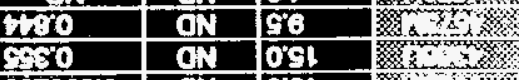

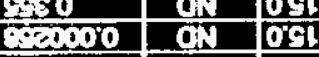
पt an 6 al

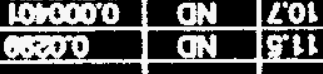
xin 
Table 2. K West Canister Liquid Sample Analysis Results from 222-S Laboratory. (Sorted by Canister)

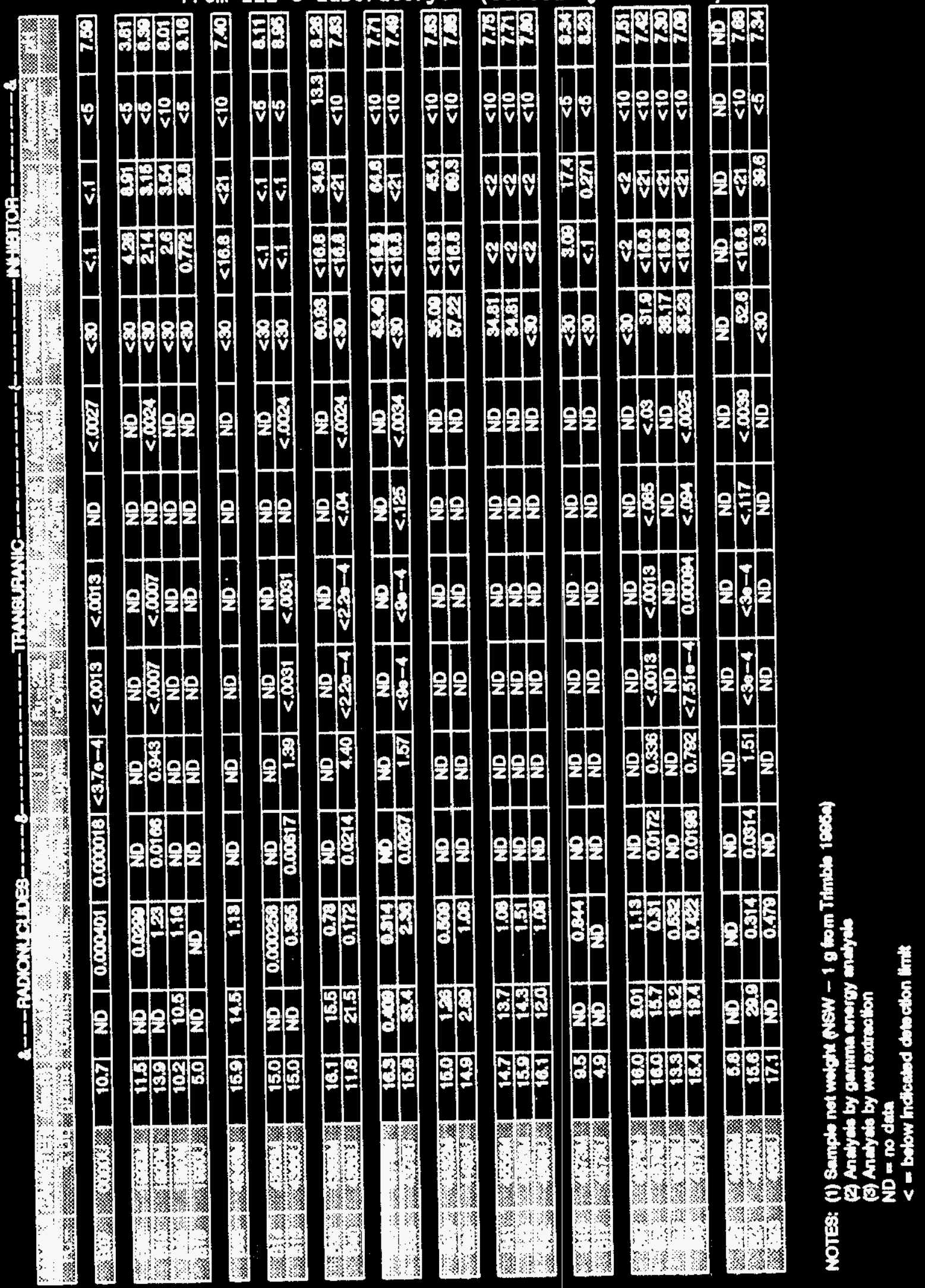


Table 3. GEA and Gas Analysis Data for K West Basin Canister Samples.

\begin{tabular}{|c|c|c|c|c|c|c|c|c|c|}
\hline ENITE & CINIIE & VMUE & Floobe0 & atrat & $c-1 \mid \overline{7}$ & $c n-157$ & $6-124$ & $\sqrt{6-86}$ & cAs \\
\hline BO & $59-10$ & (0) & $2 \sqrt{-i n}]$ & 9 & प्A & UEIPA & $\mathrm{CQ}$ & प्र & XHA \\
\hline$\therefore$ & r. & 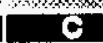 & Wo & 11.21 & 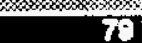 & 20 & 0.33 & & \\
\hline 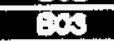 & $T \cdot T-T$ & 8 & Wo & 0.0 & & & & 3 & 72.0 \\
\hline E10 & $T+5=L$ & C & $\mathbf{T E S}$ & 13,3 & 183.0 & 13.12 & 1.2 & & \\
\hline $3 \times 6$ & $x y=1$ & C & $\bar{Y}=3$ & $10 \times 2$ & 150 & 1272 & $0 \times 1$ & & \\
\hline & & & & & & & & & \\
\hline 756 & $\mathrm{~J}=\mathrm{U}$ & 8 & $\mathrm{KO}$ & 1.70 & & & & 5.0 .6 & 0.0 \\
\hline & $\mathrm{I} \cdot \mathrm{IJ}$ & 6 & $\overline{13}$ & 504 & 48 & $\overline{a .3}$ & 0.02 & & \\
\hline & & 6 & $\mathbf{L}:$ & 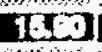 & (10) & $\mathrm{TCH}$ & $12:$ & & \\
\hline & $r=1$ & & & 19 & 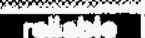 & $d n$ & & & \\
\hline & & & & & & & & & \\
\hline & C5 & G & $\mathrm{W}_{0}$ & exd & & & & 27 & 6.7 \\
\hline & & 6 & 73 & IS. & 0.010 & 0.001 & 0.00013 & & \\
\hline & & 6 & 70 & 4.7 & 20 & 0.9 & 0.016 & $<.8 .8$ & 7.7 \\
\hline & U & 8 & Ii) & e.SI & & & & 3.8 & 72.7 \\
\hline & $=\mathrm{U}$ & C & $\overline{\mathbf{M}}+3$ & 1001 & 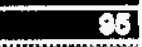 & $\overline{6.33}$ & 0.03 & & \\
\hline & & & & & & & & & \\
\hline & 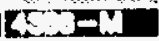 & 8 & $\mathrm{KO}$ & 200 & & & & 19 & 88.4 \\
\hline & 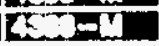 & C & ras & 106 & $\overline{203}$ & 16.56 & 1.6 & & \\
\hline & & & & 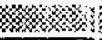 & 60 & 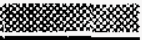 & & & \\
\hline$n$ & {$[5-J \mathbf{J}$} & 8 & 110 & 210 & & & & 23 & 98.6 \\
\hline$B$ & 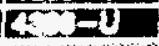 & G & $\mathrm{VIZ}$ & 11.01 & 5 & 2.67 & 1.60 & & \\
\hline & & 8 & $\mathbf{Y Z}$ & $1 C X$ & & 0.13 & 0.00 & & \\
\hline & & & & & & & & & \\
\hline & $K: 1=1$ & E & $\mathbf{B}$ & ICAI & 0.0 & Er.SB & 3 & & \\
\hline & 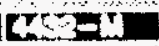 & C & $\mathrm{YZZ}$ & IRCI & 20 & 1.53 & 0.11 & & \\
\hline & & & & $4 \times x$ & 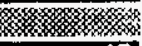 & & & & \\
\hline 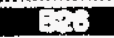 & $r=3$ & $\theta$ & $\mathrm{Y} 33$ & 1401 & 46 & 3.02 & 0.27 & & \\
\hline & w & & & & & 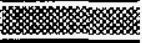 & & & \\
\hline EI & $4: 3-1)$ & 8 & $\mathrm{MO}$ & 1.08 & & & & 43 & 94.5 \\
\hline ES & $4-12$ & C & 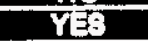 & 14.74 & 200 & 16.60 & 1.3 & & \\
\hline 8 & $t^{\prime}+x^{\prime}$ & 6 & $Y=3$ & ICA & 20 & 16,70 & 1.4 & & \\
\hline & & G & 42 & I0.1 & $T$ & 11.2 & 1.8 & & \\
\hline EIIL & $r x=r$ & $\theta$ & $\mathrm{V}=\mathbf{3}$ & $2 \times 1$ & 47 & 4.7 & 0.51 & & \\
\hline & & & & & & & & & \\
\hline [1] & $r u=0$ & 6 & 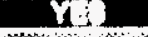 & & 3 & QD] & 0.10 & & \\
\hline & 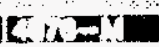 & 0 & is & {$[x \div]$} & 11 & $\mathrm{QSt}$ & 1 & & \\
\hline & $r \quad x=J$ & $G$ & $y=1$ & 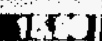 & 20 & $1 \mathrm{~T}: 0$ & 1.6 & & \\
\hline 3 & $C ; \sigma=J$ & 8 & $Y 13$ & IXI & दर्ण & ESal & 1.9 & & \\
\hline$n$ & 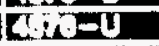 & C & $\bar{Y}=5$ & 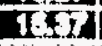 & ED & 21.47 & 10 & & \\
\hline 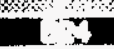 & $K \quad T=V$ & 6 & $\mathbf{Y i}$ & $\mathbf{T L I}$ & {$[01$} & CEST & 3 & & \\
\hline 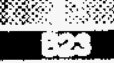 & $c-x=0$ & 6 & $\mathrm{Y} 33$ & T7.15 & 10.5 & ors & 0.12 & & \\
\hline
\end{tabular}

NOTES: (J)C - CENTER, 8 - SIDE VMVE

(2) hetrity por not wit

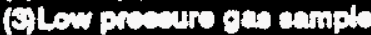

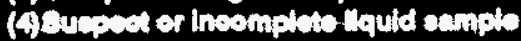




\subsection{REFEREKKES}

Harris, R. A., 1995, "Sampling and Analysis Plan for Canister Liquid and Gas Sampl ing at 105-K West Fuel Storage Basin," MHC-SD-SNF-PLN-004, Rev. 0, Westinghouse Hanford Company, Richland, Washington.

Miller, G. L., 1995, "Analysis of K West Basin Canister Liquid Samples," WHC-SD-WH-DP-138, Rev. O, Westinghouse Hanford Company, Richland, Washington.

Riedel, E. F., 1995, "Radionuclide Characterization of 105-K West Spent Fuel Storage Cantster Samples," Special Analytical Studies, Ow631-95-0717-1, Westinghouse Hanford Company, Richland, Washington.

Trimble, D. J., 1995a, "Data Compilation Report: Gas and Liquid Samples from $K$ West Basin Fuei Storage Canisters," WHC-SD-SNF-DP-001, Rev. 0 , Westinghouse Hanford Company, Richland, Washington.

Trimble, D. J., 1995b, "K West Basin Liquid/Gas Sample Data Analysis," WHC-SD-SNF-ANAL-007, Rev. 0, Westinghouse Hanford Company, Richi and, Washington. 
WHC-SD-SNF-DP-003, Rev. 0

A P PE M D I X A

LETTER, E. F. RIEDEL TO D. J. TRTMBLE, "RADIOMLLIDE CHAHCTERIZATION OF 105-K UEST SPENT FUEL STORACE

CMISTER SH CLES, " SPECTH NALYTICAL STUIES, JULY 17, 1995,

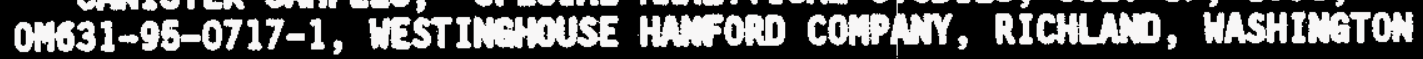


From: Special Analytical Studies

Phone: $\quad 373-2190 \quad 53-90$

Date: July 17, 1995

Subject: RADIONUCLIDE CHARACTERIZATION OF 105-KW SPENT FUEL STORAGE CANISTER SAMPLES

To:

D. J. Trimble L5-01

cc:
G. R. Blewett
$53-90$
R. A. Harris
L5-01
L. L. Lockrem
R. P. Omberg
$53-90$
EFR:LLL FI1e/LB

OM631-95-0717-1

Attached is the report "Radionuclide Characterization of 105-KW Spent Fuel Storage Canister Samples," requested in work order number 847663 .

Special Analytical Studies look forward to performing future work for your geoup.

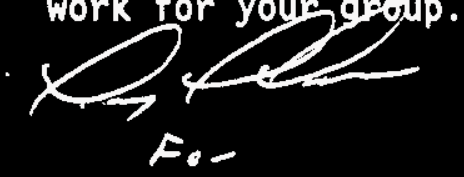

Dr. E. F. Riedel

Principal Scientist

mai

Attachment 
WHC-SD-SNF-DP-003, Rev. 0

\title{
ATTACHMENT
}

\section{RADIOHUCLIDE CHAMATERIZATION OF 105-KN}

SPETT FUEL STORAGE CAMISTER SAMPLES

Prepared by Special Analytical Studies

Hanford Technical Services

\author{
July 17,1995 \\ Consisting of 54 pages, \\ including cover sheet
}


Gamba-ray energy measurements have been made on a number of samples taken from the undemwater spent fuel storage canisters in the 100-KH Basin. The measurements were undertaken to characterize the nature of the radioisotopes present in the aqueous samples. In addition, gas samples were analyzed for the presence of krypton-85. The data show that the most common isotope found in the canisters is 1 it Cs. Krypton-85 was found to be present in some of the gas samples. Specific results are presented and discussed in the main body of this report.

\subsection{BACKEROULID}

The 105-KH Fuel Storage Basins were constructed as a part of the 100-K Production Reactor project between 1952 and 1954. The KW Basin is used for the storage of encapsulated spent fuel primarily from the 100-N reactor. At the east side of the basin pool is the Neasel Pit where the fuel canisters were sampled. Each canister consists of two barrels, and each barrel contains up to seven $N$-Reactor fuel assemblies. Canisters are of two designs, the MK I and the MK II.

Fuel was. loaded into the canisters at $100-N$, the lid installed, potassium nitrite added, and nitrogen gas added before the canisters were sealed. Currently, canisters are moved to the Weasel Pit before sampling. All sampling is being performed underwater. Separate liquid and gas samples are taken from different ports on each canister. All work other than the sample analyses was performed by Operations Personnel. Samples were analyzed by Special Analytical Studies (SAS) personnet.

Normal radiation work practices were used to meet Westinghouse Hanford Company (WHC) requirements. All sampling requirements were directed by the letter of instruction (LOI) (Reference 1) froml Spent Nuclear Fuel Evaluation (SNFE) and the Sampling and Analysis Plan (SAP) (Reference 2). Work was performed under work order $\$ E 47663$ issued by SNFE to SAS.

\subsection{INSTRUMENTATION AND MEASURENENT METHODOLOGY}

The gama-ray spectroscopy system consisted of a detector and a nuclear spectroscopy systen. The detector used was a shielded $30 \%$ p-type hyper pure intrinsfe oermanium (HPGe) detector. Btas power was supplifed to the dotector through the use of an MOMAD-Pluslportable spectroscopic system. The Momb-Plus unit also contained the signal amplifier that was used, as wall as the multichannel analyzer (MCA). The MCA converts analog input signals from the anplifier to digital output signals to form a spectrum whose photopeaks and other counting distributions correspond to signals of specific gamma-ray energies recorded by the detector. Final results; the gamma energy analysis (GEA), were obtained using a combination of the software packages MAESTRO ${ }^{2}$ and OSQ ${ }^{+3}$ along with EXCEL"

An integrated spectrometer, the NomaD-plus portable spectroscopic systen with a 486-66 computer was used for data collection and data reduction. An IT Hanford-supplfed batch file was used to 
automate and speed data reduction and reporting. WINBATCH 5 software was used for this purpose. The progran MAESTRO was used for all data collection. The program OSQ was used for all data reduction. Final spectral results for each sample were tabulated and reported in the form of an EXCEL spreadsheet immedtately after each sample was analyzed.

A set of standard geonetries were established for data collection. Because high activity samples were a possibility, parameters were established for lead shielding. Results were modeled using the progran Microshield, and compared with actual laboratory measurements for parameters such as lead shielding, distance, and the steel sleeve. Results of these comparisons proved to be within the standard errors of the measurements.

\subsection{GNHA-RAY ASSAY}

All data were collected using an 30\% hyper-pure germanium detector approximately 2 inches in diameter. The detector was cooled with liquid nitrogen, and was contained in a PopTops capsule with an all-attitude cryostat. The detector was shielded around its sides by wrapping approximately 2 inches of lead sheeting with an internal single turn each of copper and cadmium sheeting. The detector was uncollimated. Lead bricks were placed around the detector to further shield it from as much background radiation as possible.

\subsection{CALIBRATIOK METHODOLOGY}

Aqueous radiological standards produced by Amersham Corporation were measured, and the spectral data collected were used to determine the efficiency of the detector and counting electronics used for the KW-Basin measurements. Three different standards were measured, each of which contained several different isotopes. Thirteen separate spectral photopeak energy values were obtained fron these isotopes. The total energy range of these isotopes were representative of the total energy range of the isotopes actually measured at $\mathrm{KW}$ Basin. All results from these data agreed within a total error of less than $4 \%$. All these data wre fitted to a fifth order loglog polynomial equation by the method of least squares. The efficiency file created from the sumation of these spectral masurements was used in the calculation of total activity for all sample tubes masured at KH-Basin. The standards were measured at a distance of eight inches from the detector.

Two other aqueous test tube standards were prepared by the 222-SA Standards Laboratory for calibration purposes. Both tubes contained aliquots of the certified ${ }^{3}$ Cs standard solution. One tube was housed in a stainless steel sleeve (prototypic of the sample tubes used for actual sampling) and was supplied by SNFE. The other matching glass test tube had no outer stainless steel sleeve. Both test tubes were masured individually, at the same distance from the detector. The difference in ${ }^{13 y} C_{5}$ values obtained for the two standards yielded the attenuation value caused by the stainless steel sleeving. This value was $9 \%$, and was factored into all measurements at the 
basin to negate the effects of sample attenuation caused by the stainless steel sleeving. This value also agreed with Hicroshield computer modeling that was performed as a cross-check for this value.

The ${ }^{137}$ Cs test tube standards were also measured at various distances away from the detector to determine the correct distance-to-count-rate correction factor that was used for samples that were too active or inactive to be properly measured at the efght inch initial calibration distance. These correction factors were applied as appropriate to nomalize all reported results back to the initial eight inch calibration distance. Samples were counted at one of the following distances from the detector: $2-, 4-, 8-, 16-$, or 48-inches. The percent correction factors are included in ali the resuits shown in Table 1 and on the data sheets contained in Appendix A.

Varying amounts of lead attenuation was placed between the detector and a ${ }^{137}$ Cs source to determine attenuation values before beginning bas in sample measurements. These attenuation values were to be taken into account in the event that samples supplied to the MLT that were too active to be properly measured at the 48-inch maximum standoff distance without using lead attenuation. Microshield calculations also agreed exactly with these determined attenuation values. In actuality, no lead attenuation was required to make any of the necessary measurements throughout the project.

A filter paper check-source was counted on the end cap of the detector at least once a day to measure any posstble shift in detector efficiency. Measurement of the check-source was also done in conjunction with all calfbration measurements for the same reason. Measured values throughout the project agreed very closely and showed no signiffcant changes in detector effictency (see Table 2).

\subsection{BALANCE}

Sample tubes were weighed on a top loading balance capable of weighing to the nearest 0.01 gram. All test tubes were first tared with their associated plastic sleeves and tle straps, and then, wetghed with the sample when delivered by $K$ Basin operations personnel. Calibration of the balance was checked dafly using a set of callbrated standardized weights.

All samples weighed between 100 and 120 grans. The applfed calfbration range was from 100 grams to 115 grams in 5 gran intervals. Callbration of the balance was nomally checked in the morning, but never less than once a day. The error never exceeded 0.02 grans. Results were reported to the nearest 0.01 gram. By direction from SNFE, the corrected net watght is the net weight minus 1.00 gram. 


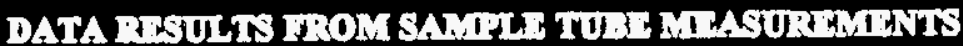

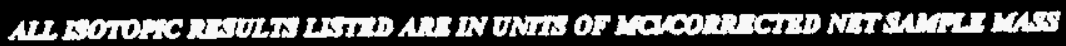

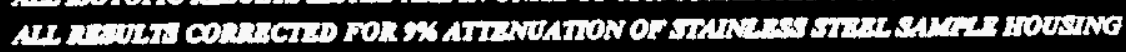

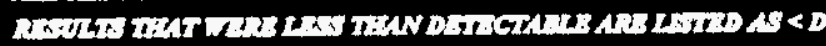

\begin{tabular}{|c|c|c|c|c|c|}
\hline$\frac{\text { Menth }}{\text { wh }}$ & 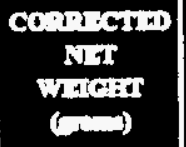 & 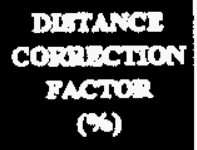 & $\begin{array}{c}\text { Pres } \\
\text { (moriotid) }\end{array}$ & 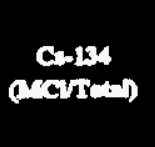 & 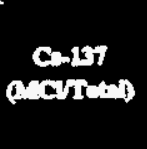 \\
\hline 141 & m?: & 175 & $<D$ & $<D$ & $<$ \\
\hline $\mathrm{H}^{\mathrm{s}}$ & 104 & 30 & $<D$ & $50: 14$ & $7 x=1$ \\
\hline$x^{\infty}$ & 0 & 121 & SI:? & $19:-2$ & $1.2 i=4$ \\
\hline Ney & 0 & 125 & $2.2 \pm 10$ & 1280 & $<D$ \\
\hline \pm & $3 \pi$ & 312 & $<n$ & 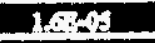 & $25 \leq 3$ \\
\hline$x^{2}+x_{2}=$ & 0 & 12s & $3.11-43$ & 2s:en & $18-4$ \\
\hline $1 ! 9$ & 27 & 12I & $<D$ & $<D$ & $<n$ \\
\hline Te. & 0 & 124 & $\angle D$ & $30 \times 4$ & 620 \\
\hline 14 & 0 & 124 & $\leq \mathrm{D}$ & $712=2$ & nxen \\
\hline $14+12$ & nes & $3 m$ & $\leq D$ & $12: 43$ & $21+4+201$ \\
\hline 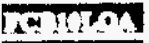 & $12 \times$ & 39 & $<n$ & 12193 & $1+2=1$ \\
\hline *alugis & 128 & 30 & $<D$ & $1.27,0.13$ & $1 \times 1: 02$ \\
\hline $14+1991$ & 0.45 & 124 & $\mathbf{s D}$ & $<D$ & $<D$ \\
\hline Neress & $a n$ & 125 & $<D$ & 3.4800 & $6.1:-09$ \\
\hline anten & $13 \times 5$ & 125 & $\leq D$ & $13: 21$ & Le:CS \\
\hline Letith? & 1602 & 340 & $<D$ & $5.3:-44$ & $2.51: 0+2$ \\
\hline$x+25 \times n$ & 84 & 3.4 & $\leq D$ & $3.18 \propto$ & $4 \pi: M$ \\
\hline anthen & 3.25 & 100 & $<D$ & Lisea & $380-2$ \\
\hline aering & 48 & 19 & $<D$ & 202 & 4.58 \\
\hline acires & $2 x$ & 30 & $<D$ & 8,420 & $1,3:-01$ \\
\hline$x=210$ & $4 x$ & 124 & $\leq D$ & 1.51915 & $2: \leq 04$ \\
\hline$x=211 x$ & 470 & 100 & $<D$ & $3 \&: 05$ & 8.0 .03 \\
\hline semprest & 1.49 & 125 & $\leq 1$ & $332: 05$ & 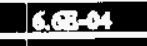 \\
\hline tenzon & 16.13 & 100 & $<D$ & 1.23 .04 & $1.0 \times 1002$ \\
\hline ixamon & 16.13 & 100 & $<D$ & .11?:05 & $1.679:-12$ \\
\hline tan:on & 16.13 & 100 & $<D$ & 9.2809 & $2.605:-02$ \\
\hline Aang & 3697 & 300 & $\leq \mathrm{D}$ & $300:-03$ & $52: 01$ \\
\hline Eenig & 1003 & 300 & $\leq D$ & 2.1:-ex & 20.10 \\
\hline$x+19$ & 12x & 3 & $<D$ & 21246 & $4 x=2$ \\
\hline 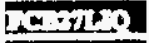 & 82 & 129 & $<D$ & SA:DT & 690 \\
\hline Xampo & 24 & 125 & $\leq D$ & $<D$ & $4.2 \leq 2$ \\
\hline Landan & $15=5$ & 10 & $<D$ & $38: 05$ & $7 \times-10$ \\
\hline I0:2ho & Mx1 & $3 n$ & $\mathrm{sD}$ & $3.8 \times 98$ & 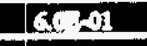 \\
\hline Lesse? & 0 & 125 & 4820 & $\angle D$ & 4200 \\
\hline$x_{1}^{2}=9$ & 1417 & 129 & $s D$ & $41:=7$ & $21 \div 2$ \\
\hline$x^{2}=a_{1}=3$ & 1 & 125 & 153 & ses:ot & $1,2=1$ \\
\hline $2=$ & 161 & 125 & 2218 & $\leq D$ & $x+=13$ \\
\hline xatim & 149 & 30 & $s D$ & 1.28 .93 & $22 \geq 0$ \\
\hline spus & 101 & 125 & $<D$ & $3: 2: 25$ & C:1 \\
\hline Wa.XIn & 15 & 30 & $\leq D$ & $1.1: 13$ & 1.93 \\
\hline$x^{4}: 121$ & 145 & 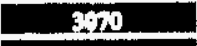 & $\leq D$ & $1 \leq 2 \leq 0$ & $2 \pi=1$ \\
\hline $\mathrm{Ea}=\mathrm{n}$ & 150 & $2 \times 10$ & $\angle D$ & 1.400 & $20:-01$ \\
\hline xatsind? & 150 & 3010 & $<D$ & $15=$ & Brs:01 \\
\hline INeren & 10.12 & 320 & $\mathbf{C D}$ & $1,1 / 2.13$ & 3.01: $=01$ \\
\hline Cand & 1911 & 210 & $<D$ & 1.21 $\geq 0$ & $a x+2=01$ \\
\hline Co:1150 & 12,24 & 30 & $\angle D$ & 1.32015 & 2201 \\
\hline $4+\sinh$ & $1 \mathrm{sm}$ & 315 & $\leq D$ & $13 \geq 13$ & $1 \times 21$ \\
\hline 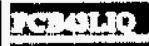 & 142 & $3 n$ & $\mathbf{S D}$ & 2.1213 & $2: 12$ \\
\hline $109 \ln 10$ & 127 & 310 & $\angle B$ & $157^{73}$ & $2 \pi i=21$ \\
\hline$x=1 \times 10$ & 247 & $2 \pi$ & $\mathbf{C D}$ & $18 \times 3$ & $3 \times 12$ \\
\hline
\end{tabular}

\begin{tabular}{|c|c|c|}
\hline 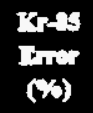 & $\begin{array}{l}\text { Cork } \\
\text { (w) }\end{array}$ & $\begin{array}{l}C-137 \\
\text { Dis } \\
(x)\end{array}$ \\
\hline $\mathbf{N A}$ & $\mathrm{NI}$ & $\boldsymbol{B} \boldsymbol{M}_{1}$ \\
\hline $\mathbf{N}$ & 6 & 3 \\
\hline 7 & $C$ & 2 \\
\hline 7 & 46 & $\mathrm{~N} / \mathrm{a}$ \\
\hline $1 M$ & 1 & 7 \\
\hline 9 & 17 & 10 \\
\hline $1 \%$ & 27 & $\boldsymbol{N} \boldsymbol{\Lambda}$ \\
\hline 21 & 25 & 10 \\
\hline 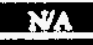 & $2:$ & 10 \\
\hline$N A$ & 8 & 7 \\
\hline $\mathbf{N A}$ & 8 & 7 \\
\hline Na & 8 & 7 \\
\hline $1 / A$ & $2 / 4$ & $\mathbf{N} \boldsymbol{\Lambda}$ \\
\hline $2 / 4$ & 23 & 9 \\
\hline $\mathrm{N} / \mathrm{A}$ & 51 & 7 \\
\hline$N / 4$ & 8 & 7 \\
\hline $\mathrm{NR}$ & 11 & 1 \\
\hline WA & 8 & 7 \\
\hline$N \Lambda$ & 9 & 7 \\
\hline$N / A$ & 9 & 7 \\
\hline $\mathrm{N} / \mathrm{A}$ & 12 & 7 \\
\hline $\mathbf{N}$ & 11 & 1 \\
\hline $\mathrm{SWA}$ & 14 & 7 \\
\hline NA & 25 & 7 \\
\hline $\mathrm{Na}$ & 12 & 7 \\
\hline $\mathrm{N} / \mathrm{A}$ & 11 & 7 \\
\hline$N / A$ & 31 & 7 \\
\hline $\mathbf{X} / \mathbf{A}$ & 19 & 7 \\
\hline$x_{A}$ & 11 & 7 \\
\hline $\mathrm{Na}$ & 3 & 7 \\
\hline$N_{A}$ & NA. & 7 \\
\hline $2 \mathrm{NA}$ & 12 & 7 \\
\hline$\Sigma / \Lambda$ & II & 7 \\
\hline 1 & $\mathrm{NA}$ & 8 \\
\hline$\sqrt{21}$ & 3 & 7 \\
\hline 7 & 16 & 7 \\
\hline 7 & W & 8 \\
\hline $2 \pi$ & 10 & 7 \\
\hline$N \Lambda$ & 2 & 7 \\
\hline 24 & 12 & 7 \\
\hline $\mathbf{I} / \mathrm{A}$ & 30 & 7 \\
\hline 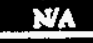 & 11 & 7 \\
\hline$N_{\Lambda}$ & 11 & 7 \\
\hline$N A$ & 11 & 7 \\
\hline $\mathrm{X}$ & 16 & 7 \\
\hline 50 & 11 & 7 \\
\hline 12 & 11 & 7 \\
\hline $\mathrm{FA}$ & 11 & 7 \\
\hline$N_{2}$ & $M$ & 7 \\
\hline$N A$ & 10 & 7 \\
\hline
\end{tabular}


Table 2. Quality Assurance Data from Daily QA Source Check.

ML ISOTOFIC QA VALUES ARE EXPRBSSED W UNTS OF nCHTOTAL SOURCE FLENAMES DXPRBSS THE DATE ON WHCH THE RUN WAS MADE

\begin{tabular}{|c|c|c|c|}
\hline QA FILENAME & Am-231 & C5-137 & Co-60 \\
\hline QA021695.80 & 2802 & 2374 & 1655 \\
\hline QA021795.S0 & 2857 & 2685 & 1632 \\
\hline CA022305.S0 & 3018 & 2701 & 1035 \\
\hline CA0303:5.50 & 2848 & 2684 & 1631 \\
\hline CA305.5.50 & 2953 & 2884 & 1636 \\
\hline CA30705.50 & 3058 & 2755 & 1618 \\
\hline QA30:05.50 & 2738 & 2605 & 1859 \\
\hline QA31305.80 & 2760 & 2712 & 1633 \\
\hline QA31405.80 & 3011 & 2783 & 1612 \\
\hline QA34505.80 & 3038 & 2702 & 1585 \\
\hline QA31805.S0 & 3040 & 2741 & 1807 \\
\hline QA31685A.S0 & 2748 & 2711 & 1627 \\
\hline QA31705.80 & 3023 & 2711 & 1603 \\
\hline CA31895.80 & 2898 & 2688 & 1584 \\
\hline QA32095.S0 & 2838 & 2639 & 1613 \\
\hline QA32005A.S0 & 2855 & 2666 & 1618 \\
\hline QA32095B.50 & 2863 & 2691 & 1614 \\
\hline CA32195A.S0 & 2785 & 2673 & 1593 \\
\hline QA32305.80 & 3123 & 2714 & 1646 \\
\hline QA32495.50 & 2970 & 2611 & 1574 \\
\hline QA32495B.50 & 3092 & 2702 & 1640 \\
\hline QA32495C.50 & 2922 & 2687 & 1618 \\
\hline QA32595.S0 & 2910 & 2578 & 1557 \\
\hline QA32685A.50 & 2943 & 2580 & 1536 \\
\hline QA32795.80 & 2977 & 2641 & 1555 \\
\hline
\end{tabular}

\section{SUMMARY}

\begin{tabular}{|c|c|c|c|}
\hline Maximum values & 3123 & 2783 & 1659 \\
\hline Minimum values & 2738 & 2570 & $\overline{1536}$ \\
\hline Averese & 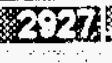 & 203 & 16 \\
\hline
\end{tabular}


WHC-SD-SWF-DP-003, Rev. 0

\subsection{STMUNO CzongTRIES}

All samples were received in rubber stoppered test tubes that were jacketed with a stainless steel sleeve. The tubes were capable of holding slightly over 16 milliliters of sample solution. After basin operations filled the tubes with either solution or gas, they were double bagged in plastic sleeving and each bag was individualiy sealed.

For counting purposes inside the Special Analytfcal Studiesnondestructive analysis SAS-NDA truck, a holder was constructed for gripping the test tubes. This holder was used for both reproductbility and to hold the test tubes securely in an upright position for analyses. A slot existed in each of the stainless steel tube sleeves and this slot was aiways positioned so that it faced away from the detector during analysis.

Corrections for lead shfelding were established using actual measurements and also using calculations. Lead thicknesses of $0.5 \%, 1-$, and 2 -inches were used. For this group of samples, no lead shielding was required for any measurements.

Standard distances of 2 inches, 8 inches, 16 inches and 48 inches were established for measurement of samples. All analyses were performed at one of these distances. The distance used was selected so as to minimize the counting dead time. Since none of the samples were too active to be counted at the maximum distance of 48 inches, no lead shielding was required to 1 imit the dead time. The 8 inch distance was selected as the standard distance and all other measurements were corrected to this distance.

Correction for absorption of the steel was determined experimentally and this attenuation was veriffed by modeling using the progran Microshield. The attenuation due to the sleeving was calculated to be $9 \%$. All results were corrected for this factor.

All samples were centered in front of the detector face both horizontally and vertically. Allowances for differences in sample volumes were taken into account by centering sample tubes in front of the detector relative to the amount of sample present. A number of shims were made and used for this centering activity. A reference table of sample volune (weight) verses shim heights was constructed and used for all measurements.

\subsection{RESULTS}

The results obtained are sumarized in Table 1 and presented in detafl in Appendix A. Results are presented in the units of alllicuries of activity per total sample (nci/total). Errors are given at the two siga level for all analytes found. The analyte list presented represent those commonly found around the Hanford Site. All spectra were examined to determine if any other analytes were present (none were). Corrected net sauple weights (in grams) are also presented. Net sample weight is defined as gross tube weight with saiple, minus tube weight without sample, minus one gran. 
The individual report sheets also list the distance from the detector in inches with the factor used to correct that distance back to the standard distance of elght inches. The customer identification is also listed on the sheet. In Table 2, the sample identification is by the customer identification with a two or three letter adder that identiffes whether the sample is liquid, gas, or duplicate. For example, FCB23 has three adders; namely LQ for liquid, LQA for duplicate recounting, and $L Q B$ for a third duplicate recount.

Check standard results are summarized in Table 2. This check standard contained anerictum, cesium, and cobalt. The quality assurance check is identified by a QA followed by the six number date (two numbers each for the month, day, and year). Since sometimes, more than one QA measurenent was made on a given day, only one number was used for the month and a letter was added for differentiation.

Results showed that the tubes containing gas were usually active with krypton-85 while the liquid samples were usually active with ${ }^{37}{ }^{C s}$, as was expected.

\subsection{ERRORS}

All errors are reported at the two sigma level. This means that the correct (or true) value should be within the reported limits $95.5 \%$ of the time.

The reported errors come from three sources. Two of these are mathematically calculated and the third is scientifically estimated, in other words, a scientific best guess.

The first source of error is derived from counting statistics. This number is the square root of the total peak counts (that is peak area plus background) plus the background counts. (Background is actually added in twice, but it is also measured twice.) Thus, this number is always dependent on the total number of counts observed in the photopeak.

The second source of error is associated with the least squares fit of the efficiency calibration. Data is collected on a number of standards and is fit using the least squares technique to a fifth order polynowial. This type of error is dependent on the photopeak energy, since this error is not a constant. In this case the error associated with the ${ }^{137}$ Cs photopeak is $1.62 \%$ while that associated with the ${ }^{85} \mathrm{Kr}$ photopeak is $1.65 \%$.

The third source of error is all the other uncertainties associated with the data collection activities. This error is estimated by the scientist, in other words it is a best scientific guess. In general the factors which contribute to this error include the following:

- Ceometry--how repeatable is the measurement, due to factors such as positioning and distance.

- Reported uncertainties in the standards used for calibration. 
- Errors associated with the sampling process.

Estimate of the overall uncertainties in the reported nuclear properties such as the branching ratio and the actual energy of the gama ray that is emitted. This information is avallable in the ifterature.

For this spectfic example, the principle contributions are from the geometric effects and the uncertainties in the standards used for calibration.

Errors one and two are calculated by the software. For these data measurements a value of $3 \%$ was used for the third type of error.

\section{0 conclustoH}

The techniques used in this project resulted in rapid presentation of analyte activities to SNFE at K-Basin. This methodology should be useful in stifiar future operations involving the fuel casks.

A number of the samples were analyzed by the 222-S Laboratory. Field results should be compared to the laboratory results.

From a data collecting perspective a few jmprovements can be made. The software was rather slow and often represented the largest portion of the time required to present the results to the client. This can be improved by replacing the WINBATCH driver with a program written in Visual Basic. The sample holder was also cumbersome and should be redesigned for easier operation.

Some of the samples were very low in activity and it is possible that basin liquid was collected rather than the desired material. If a radioactive tracer were added to a single container it would be possible to determine if. the sampling device was working correctly. It is suggested that this be done for future operations. Measurement of this test container could be done every fifth or tenth measurenent and would confirm that the sampler was working correctly.

We would like to acknowledge the help and assistance provided by Mr. Brian Keele. He developed the WINBATCH progran and provided other invaluable assistance.

\subsection{REFERENGES}

1. LOI: IMSTRUCTIONS FOR GAMMA ENERGY ANALYSIS OF K WEST BASIN CANISTER SAMPLES, D. J. Trimble, March 6, 1995.

2. SAP: "Sampling and Analysis Plan for Canister Liquid and Gas Sampling at 105-K West Fuei Storage Bas in," WHC-SD-SNF-PLN-004, ReV 0, Westinghouse Hanford Company, Rtchland, Washington, Harris, R. A.

3. "Dosk Instructions for KW-Bas in On-site Sample Analysis", 0M630-95-008. 
WHC-SD-SNF-DP-003, Rev. 0

APPENDIX A 
CAMMA-RAY ENERGY ANALYSIS REPORT

Spocial Anotiled studes

Wentritioum Honford Compary

115.61 = Sample Groes (o)

-103.05 = Sampie Tare (o)

-1.00 - Bampio Entrapd HBO (o)

Customer 10 Number: becin $\mathrm{H} 20 \mathrm{O} 2 \mathrm{2}$ inches 11.50 - SAMPLE NaT (0)

Laboratory ID Number: FCBO1

\begin{tabular}{|c|c|c|c|}
\hline botope & & $\begin{array}{l}\text { Corrected } \\
\text { netrotal }\end{array}$ & Error \\
\hline $\begin{array}{l}\text { Be7 } \\
\text { K40 } \\
\text { Co57 } \\
\text { Cos0 } \\
\text { Kros } \\
\text { RuRh103 } \\
\text { Sb125 } \\
\text { I129 } \\
\text { Co134 } \\
\text { Cs137 } \\
\text { Ce144 } \\
\text { Eu152 } \\
\text { Eu154 } \\
\text { Eu155 } \\
\text { Th32dau } \\
\text { U235 } \\
\text { U238 } \\
\text { U238dau } \\
\text { Np237 } \\
\text { Pu239 } \\
\text { Pu240 } \\
\text { Am241 }\end{array}$ & $\begin{array}{l}< \\
< \\
< \\
< \\
< \\
< \\
< \\
< \\
< \\
< \\
< \\
< \\
< \\
< \\
< \\
<\end{array}$ & $\begin{array}{l}1.4 e-06 \\
2.1 e-08 \\
1.3 e-08 \\
1.5 e-07 \\
3.5 e-05 \\
1.4 e-06 \\
6.0 e-07 \\
3.5 e-05 \\
1.2 e-07 \\
2.7 e-06 \\
1.2 e-06 \\
3.6 e-07 \\
3.4 e-07 \\
7.0 e-07 \\
3.3 e-07 \\
1.3 e-06 \\
1.6 e-05 \\
4.3 e-07 \\
3.9 e-07 \\
2.2 e-03 \\
3.0 e-02 \\
9.2 e-07\end{array}$ & \\
\hline
\end{tabular}

12.50\% Dimtance correction factor [100\% moans no correction] 9.00\% Attoriation (Sampia tube) $0.00 \%$ Amention (Ehild)

Activity $(m c l)$ at

Errors and MDA reported at 2 cioma

Dr. E. Fred Rrodol

$3-14-96$ 


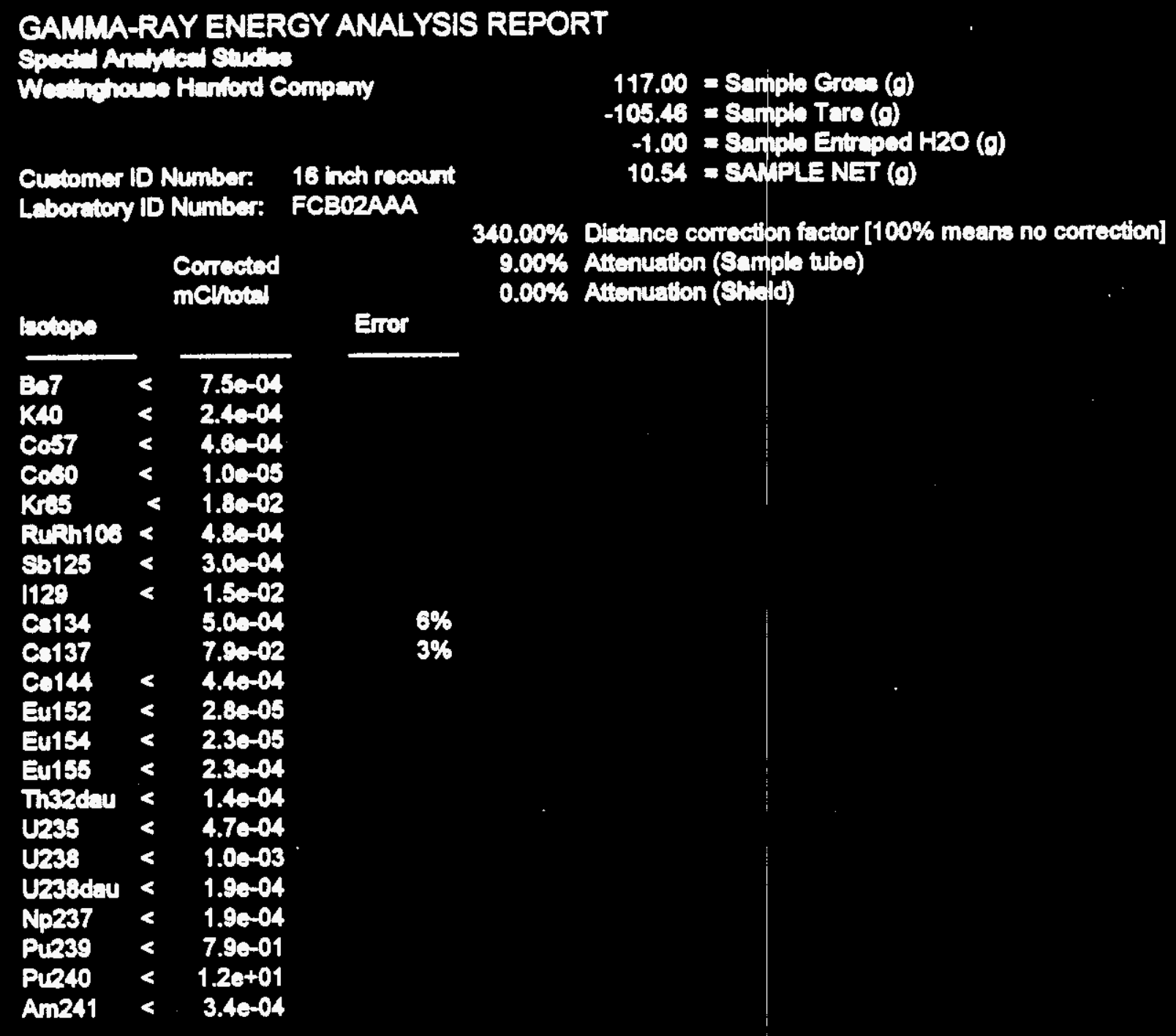

Erose and MDA reported at 2 cioma $3-15-\oplus$

Dr. E. Fred Finder 


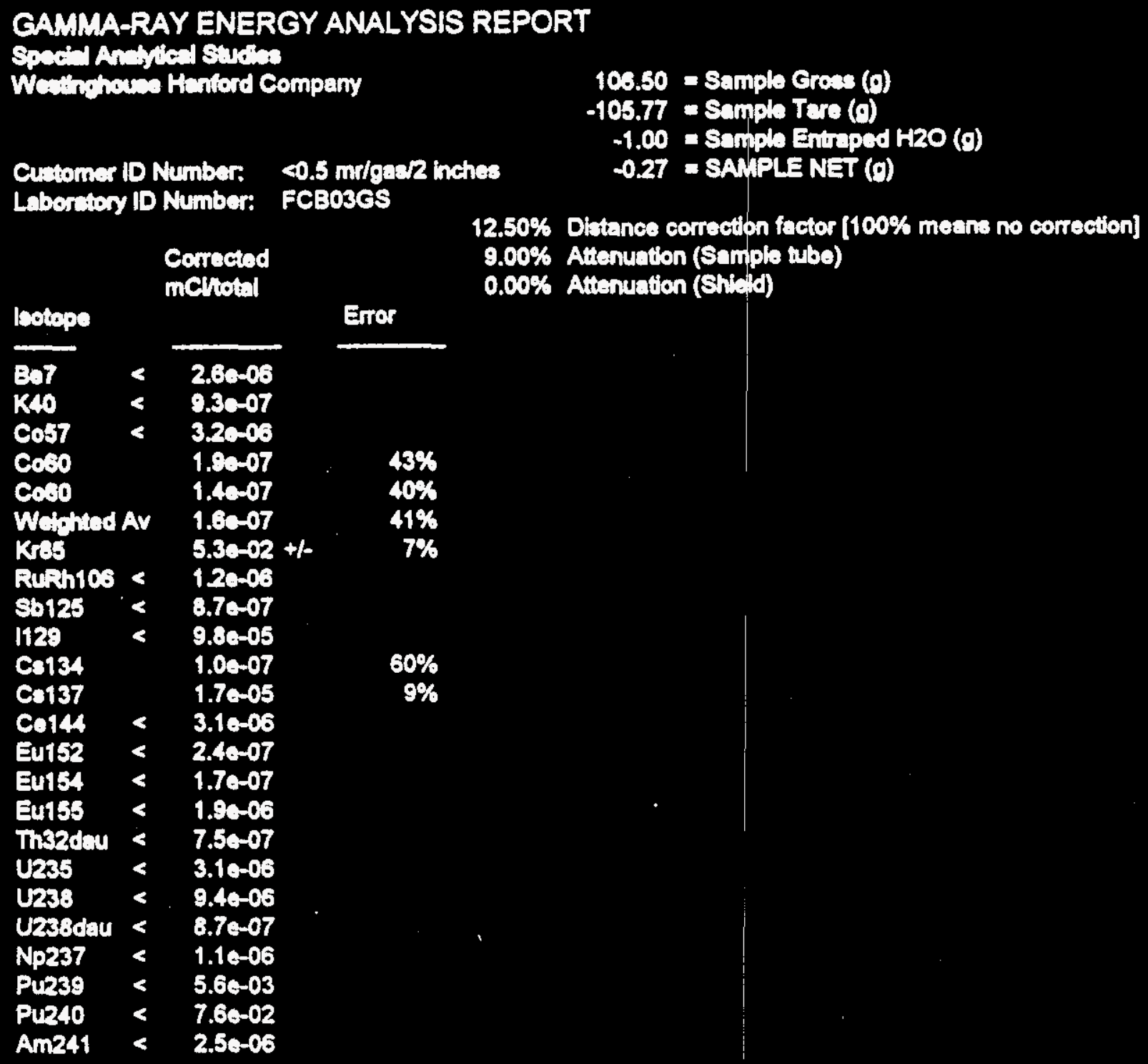

Erore and MDA reported at 2 cioma

3-16-98

Gordon Eimmit: 
CAMMA-RAY ENERGY ANALYSIS REPORT

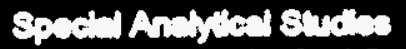

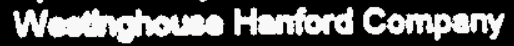

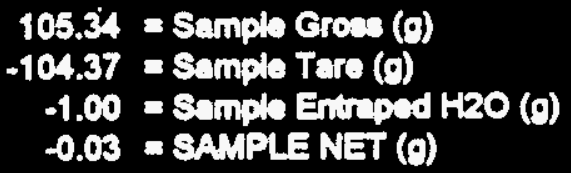

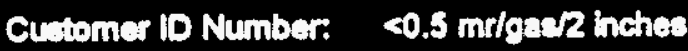
Laboratery 10 Number: FCBQ40S

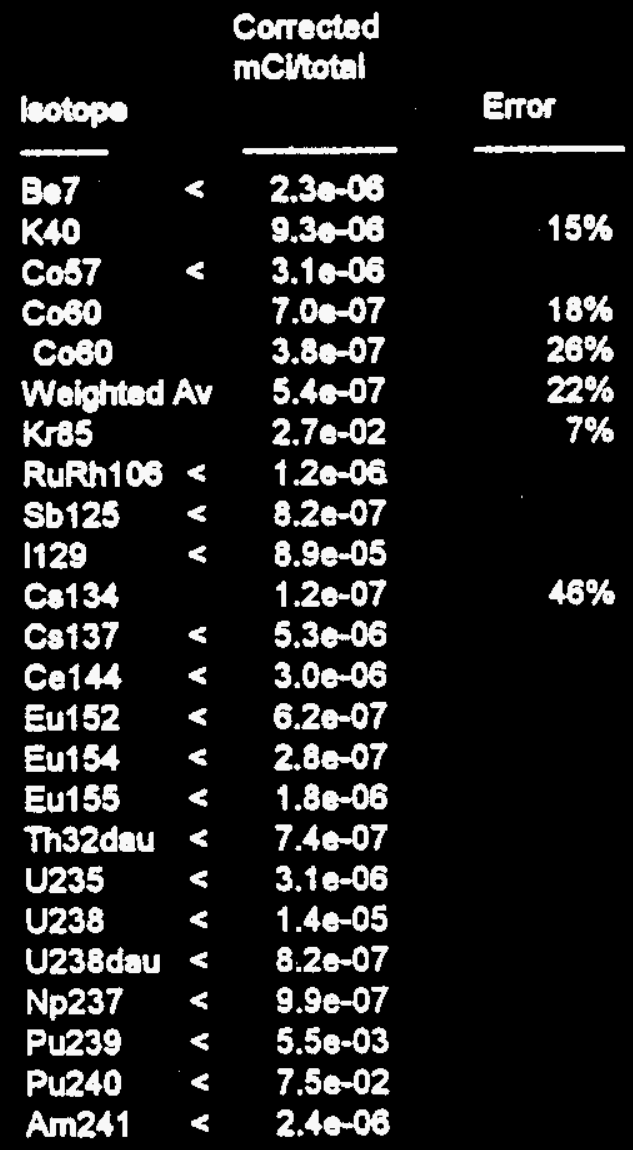

$12.50 \%$ Distance correction factor [100\% means no correction] 8.00\% Attenuntion (Sample trbe)

$0.00 \%$ Attonuation (Ehidd)

$15 \%$

$18 \%$

$26 \%$

$7 \%$

$48 \%$

Enos and MOA reported at 2 sions

$3-17-95$

Cocten Elemwit 
GAMMA-RAY ENERGY ANALYSIS REPORT

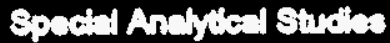

Wuninimo Heriord Company

108.48 = Sample Crose (o)

-103.91 - Sample Tare (o)

-1.00 - Samplo Entraped H2O (o)

Customer ID Number: $\quad 0.5 \mathrm{mrni} / 4 \mathrm{inches}$ 3.57 - SAMPLE NET (o)

Laboratory 10 Number: FCBOstQ

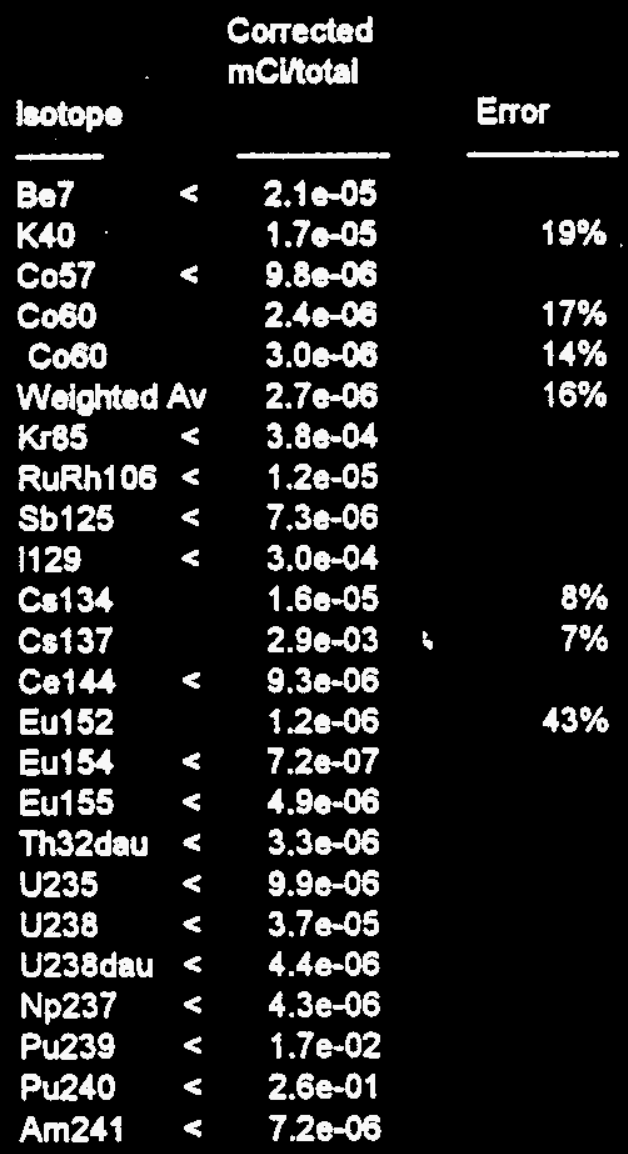

$31.20 \%$ Distance correction factor [100\% means no correction]

$0.00 \%$ Attenuation (Sample tibe)

$0.00 \%$ Attonuation (Etidd)

Enore and MDA reported at 2 signe

3-17-95

Gordon Blomat: 


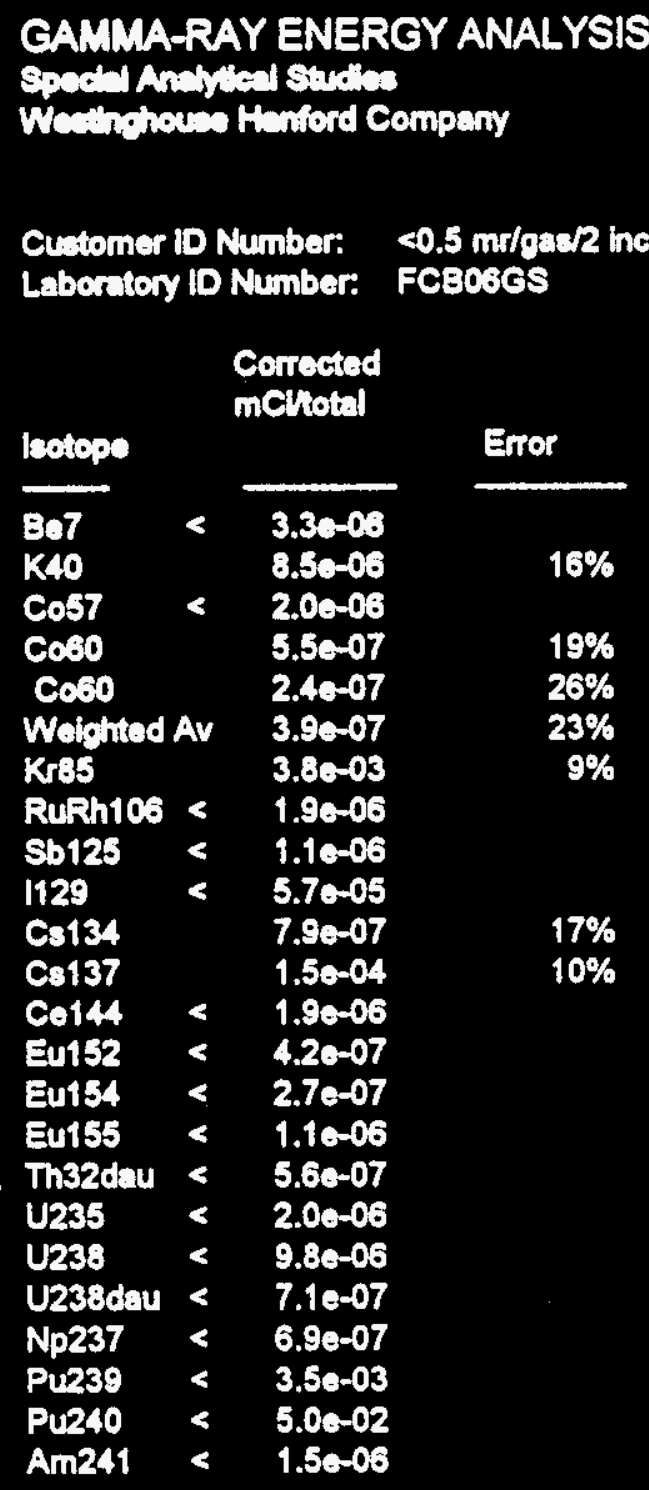

Erros and MDA reported at 2 signo

$12.50 \%$ Dlatance correction factor [100\% means no correction] $0.00 \%$ Attenuation (Sample trbe)

$0.00 \%$ Attenuation (Shisid)

$$
\begin{aligned}
105.13 & =\text { Sample Grose }(0) \\
-105.50 & \text { - Sample Tare }(0) \\
-1.00 & =\text { Sampla Entraped H2O (o) } \\
-0.37 & =\text { SAMPLE NET }(0)
\end{aligned}
$$

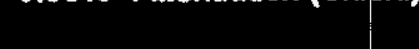

\section{$16 \%$ \\ $26 \%$ \\ $3 \%$ \\ $7 \%$ \\ $0 \%$}

Dr. E. Frod Riadel

3-17-95 


\section{CAMMA-RAY ENERGY ANALYSIS REPORT}

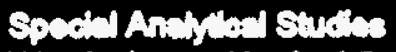

Wentionere Hanford Company

$$
\begin{aligned}
117.26 & \text { = Sample Crow }(0) \\
-103.55 & \text { \# Sample Tare }(0) \\
-1.00 & =\text { Samplo Entraped H2O (o) }
\end{aligned}
$$

\begin{tabular}{|c|c|c|c|}
\hline betepese & & $\begin{array}{l}\text { Cerrected } \\
\text { neltotal }\end{array}$ & Enor \\
\hline $\begin{array}{l}\text { Eut } \\
\text { K10 } \\
\text { Cost } \\
\text { Co60 } \\
\text { Coco } \\
\text { Kros } \\
\text { RuRhitos } \\
\text { sb125 } \\
1129 \\
\text { Co134 } \\
\text { Co137 } \\
\text { Ce144 } \\
\text { Eu152 } \\
\text { Eu154 } \\
\text { Eu155 } \\
\text { Th32dau } \\
\text { U235 } \\
\text { U238 } \\
\text { U238dau } \\
\text { Np237 } \\
\text { Pu239 } \\
\text { Pu240 } \\
\text { Am241 }\end{array}$ & $<$ & $\begin{array}{l}1.20-06 \\
7.6 e-06 \\
1.4 e-06 \\
2.8 e-07 \\
1.20-07 \\
2.8 e-05 \\
1.1 e-06 \\
4.00-07 \\
3.5 e-05 \\
1.0 e-07 \\
7.0 e-06 \\
1.3 e-06 \\
4.4 e-07 \\
2.5 e-07 \\
7.5 e-07 \\
3.4 e-07 \\
1.4 e-06 \\
1.20-05 \\
3.1 e-07 \\
3.46-07 \\
2.4 e-03 \\
3.4 e-02 \\
8.7 e-07\end{array}$ & $\begin{array}{l}17 \% \\
29 \%\end{array}$ \\
\hline
\end{tabular}

Cuntomer ID Number. $\quad 0.5$ mrilla/2 inches Laboritory ID Number. FCBO7LQ

12.50\% Distance correction factor [100\% means no correction] $9.00 \%$ Attenuation (Banple tobo) $0.00 \%$ Attonuation (Ehitid)

Enore and MDA reported at 2 sioma $3-17-95$

D. ERridrade 


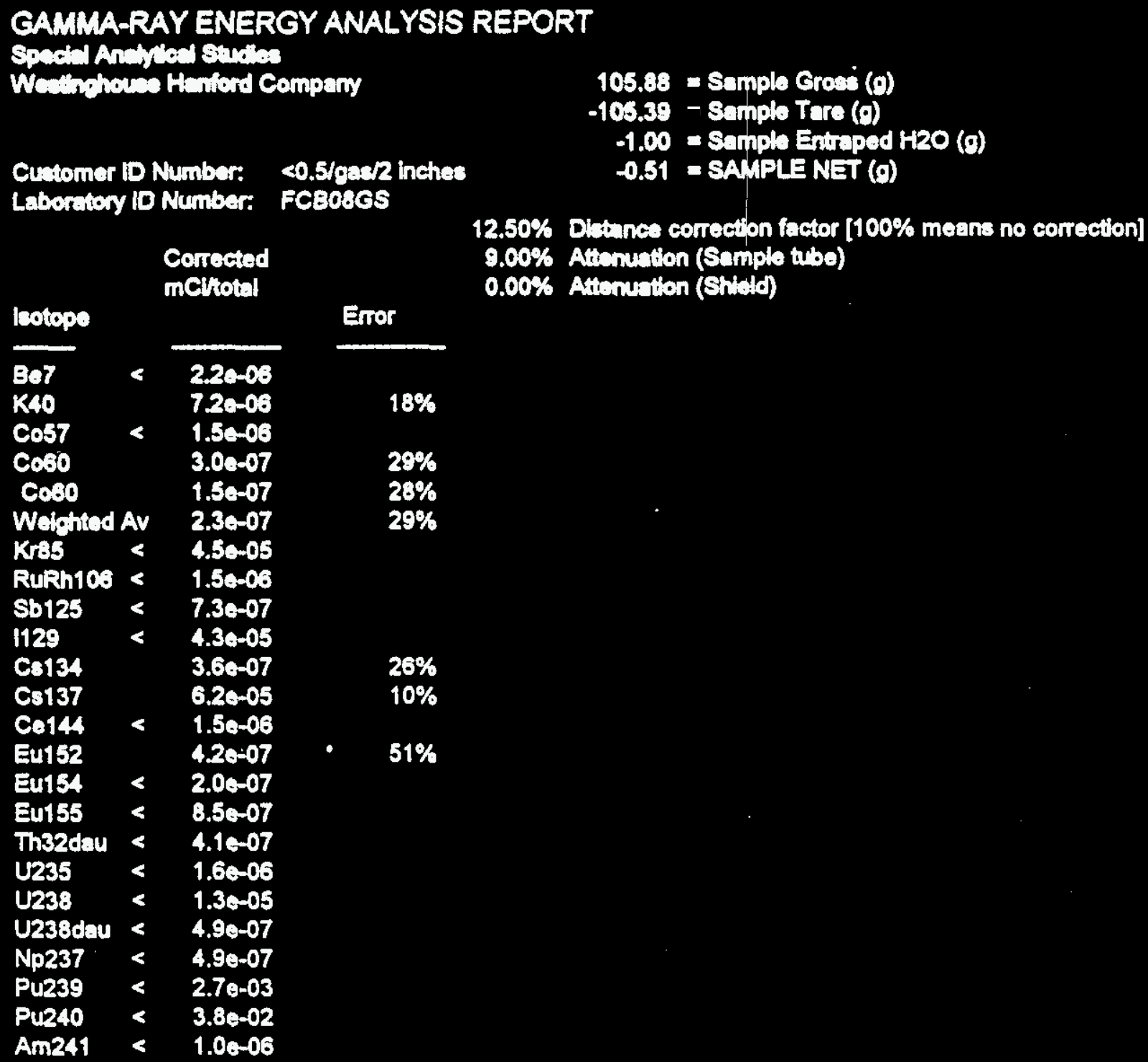

CAMMA-RAY ENERGY ANALYSIS REPORT

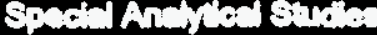

Wourime Herierd Company

105.88 - Sample Gross (o)

(a)

Laborctory io Number: FCBoses

Corrected 9.00\% Atanuation (Sample trbo)

lootoper

Enor

Bet $<22000$

K40 7.20-00

$0057<1.50-06$

Cos0 $3.00-07$

Co60 $1.50-07$

2.3e-07

$28 \%$

Kras \& 4.50m05

RuPh109 \& $1.50-06$

Sb125 \& 7.36-07

$1129<4.30-05$

Cs134 3.6e-07

Cs137 6.20-05

Co144 < 1.5e-08

Eu152 4.20:07

Eu155 < 8.5e-07

Th32day \& 4.1e-07

U235 < 1.6e-0s

$1238<1.30-05$

U238dau < 4.9e-07

$<4.90-07$

Am241 \& 1.00-08

Errore and MDA reported at 2 sioma

3-17-95

Dr. E. Fred Riodel 


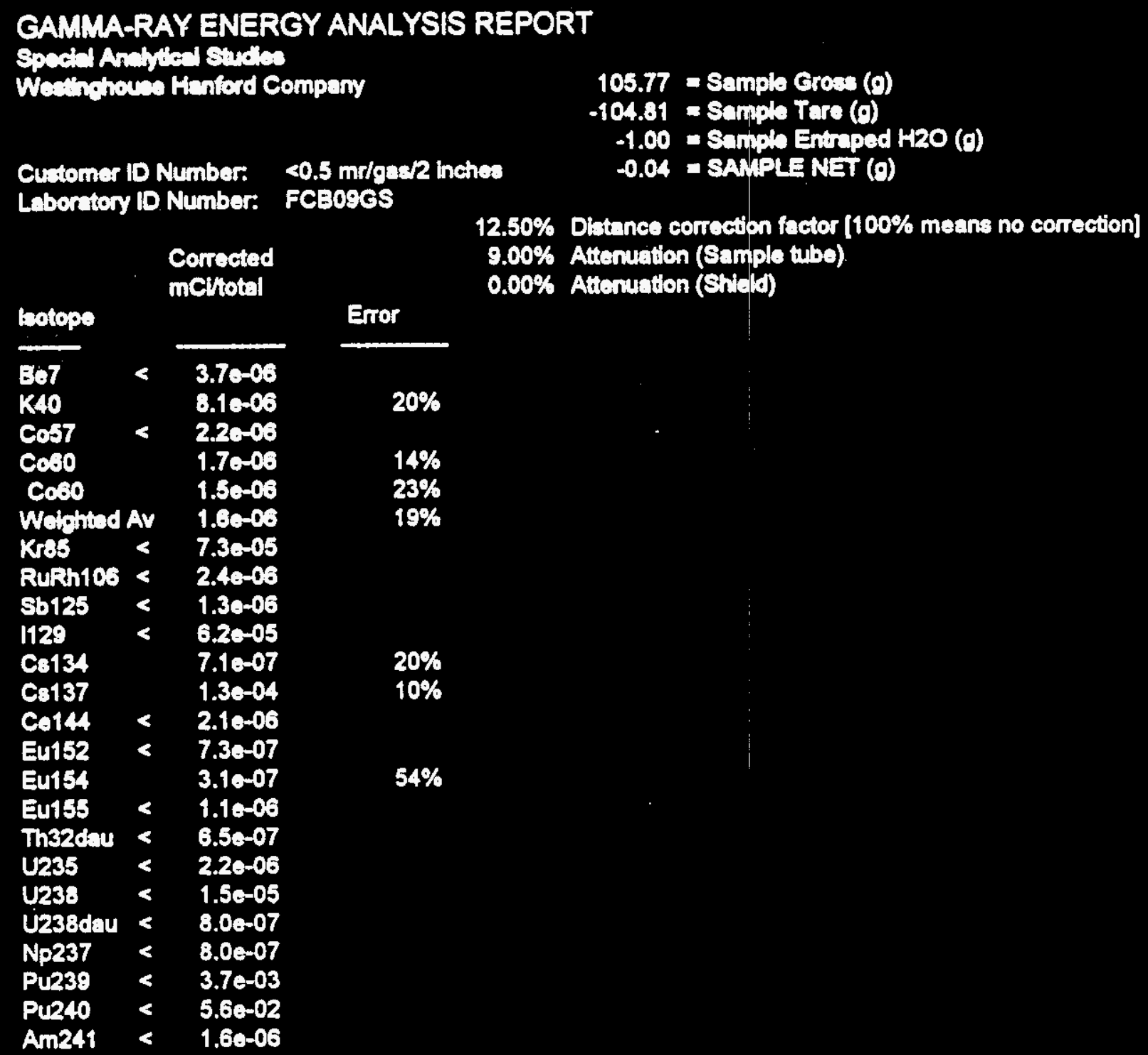

Errote and MDA reported at 2 sigme

3-17-95

Dr. E. Fred Rrod 


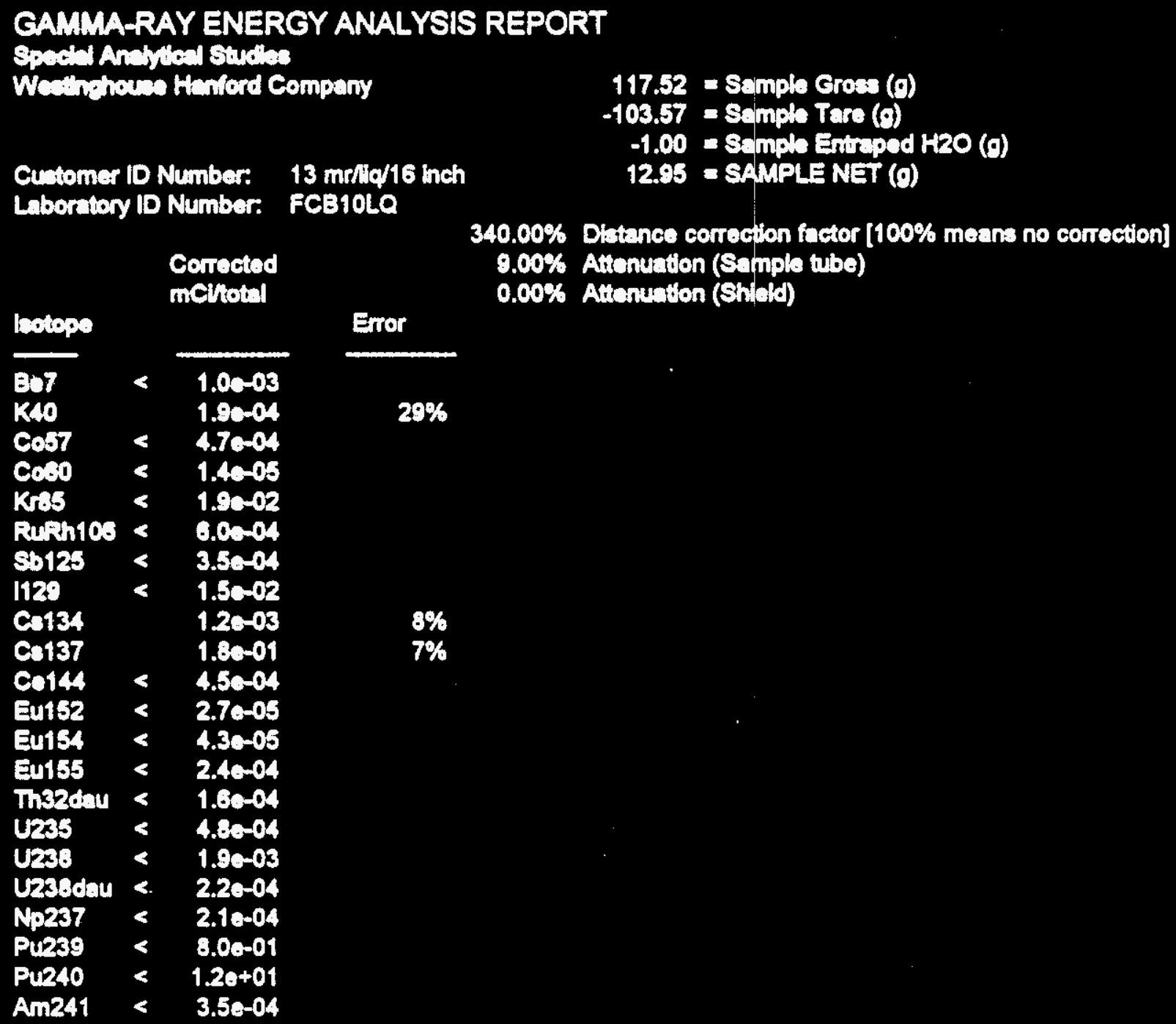

Errore and MDA reperted at 2 sigma

3-18-95

Cordin Ermi: 


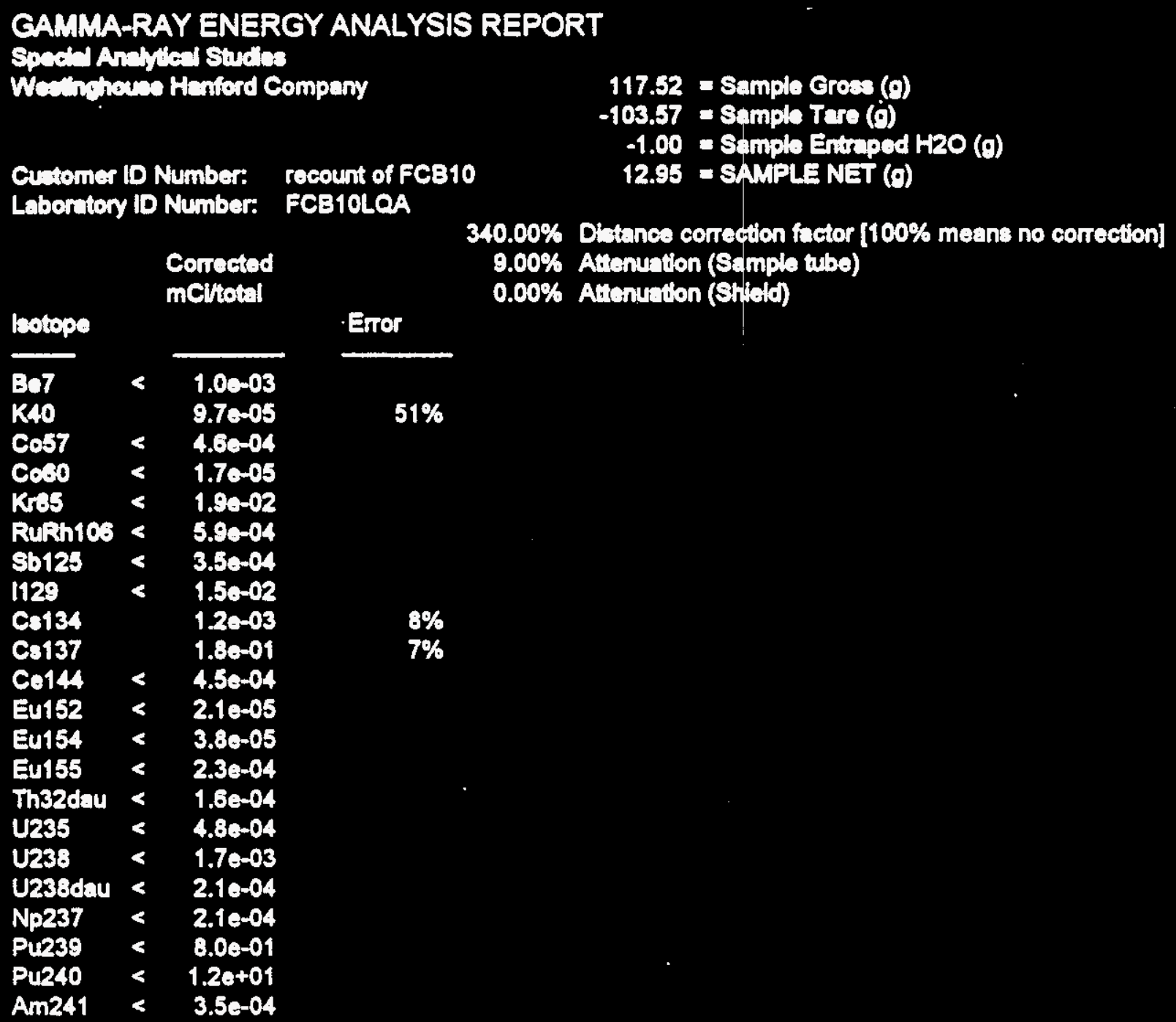

Enore and MDA reported at 2 sigma 3-10-98

Gorden Bomet 
CAMMA-RAY ENERGY ANALYSIS REPORT

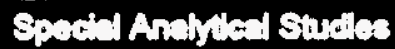

Wentistowe Hanterd Company

117.52 = Sampla Groes (o)

-103.57 - Bamplo Tare (o)

-1.00 - Sampla Entrapod H2O (o)

Customer ID Number: and recount of FCB10 12.95 - SAMPLE NET (o)

Laborntory ID Number: FCB10LOB

\begin{tabular}{|c|c|c|c|}
\hline botope & & $\begin{array}{l}\text { Sorrected } \\
\text { ncitotal }\end{array}$ & Enror \\
\hline $\begin{array}{l}\text { Ba7 } \\
\text { K40 } \\
\text { Cost } \\
\text { Co60 } \\
\text { Kus } \\
\text { Ruth106 } \\
\text { Sb125 } \\
\text { l129 } \\
\text { Co134 } \\
\text { Co137 } \\
\text { Co144 } \\
\text { Eu152 } \\
\text { Eu154 } \\
\text { Eu155 } \\
\text { Th32dau } \\
\text { U235 } \\
\text { U238 } \\
\text { U238dau } \\
\text { Np237 } \\
\text { Pu239 } \\
\text { Pu240 } \\
\text { Am241 }\end{array}$ & $\begin{array}{l}< \\
< \\
< \\
< \\
< \\
< \\
<\end{array}$ & $\begin{array}{l}1.00-03 \\
1.20-04 \\
4.70-04 \\
1.40-05 \\
1.80-02 \\
5.90-04 \\
3.50-04 \\
1.5 e-02 \\
1.20-03 \\
1.80-01 \\
4.5 e-04 \\
2.7 e-05 \\
4.70-05 \\
2.40-04 \\
1.6 e-04 \\
4.80-04 \\
1.80-03 \\
2.10-04 \\
2.10-04 \\
8.0 e-01 \\
1.20+01 \\
3.5 e-04\end{array}$ & $\begin{array}{l}8 \% \\
7 \%\end{array}$ \\
\hline
\end{tabular}

$340.00 \%$ Distance correction factor [100\% means no correction] 9.00\% Attenuation (Bample tube)

$0.00 \%$ Attenution (Bhild)

$\%$

$7 \%$

$5 \%$

ErTore and MOA reported at 2 sigma

Carden Elomett

3-18-95 
GAMMA-RAY ENERGY ANALYSIS REPORT

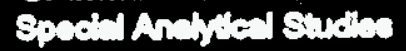

Weitherieve Henterd Company

109.20 = Sample Groes (o)

-107.75 - Sample Tare (o)

$-1.00=$ - Samplo Entraped H2O (0)

Customer ID Number: 3-2016b12 gae-2 inch

0.46 - SAMPLE NET (o)

Laboritery ID Number: feb120es

\begin{tabular}{|c|c|c|c|}
\hline botope & & $\begin{array}{l}\text { Sorrected } \\
\text { nCltotal }\end{array}$ & Error \\
\hline $\begin{array}{l}\text { Bo7 } \\
\text { K10 } \\
\text { Cost } \\
\text { Coco } \\
\text { Kros } \\
\text { Ruin10s } \\
\text { Sb125 } \\
\text { I129 } \\
\text { Co134 } \\
\text { Cs137 } \\
\text { Co144 } \\
\text { Eu152 } \\
\text { Eu154 } \\
\text { Eu155 } \\
\text { Th32dau } \\
\text { U235 } \\
\text { U238 } \\
\text { U238dau } \\
\text { Np237 } \\
\text { Pu239 } \\
\text { Pu240 } \\
\text { Am241 }\end{array}$ & $\begin{array}{l}< \\
< \\
< \\
< \\
< \\
< \\
< \\
< \\
< \\
< \\
< \\
< \\
< \\
< \\
< \\
< \\
< \\
< \\
<\end{array}$ & 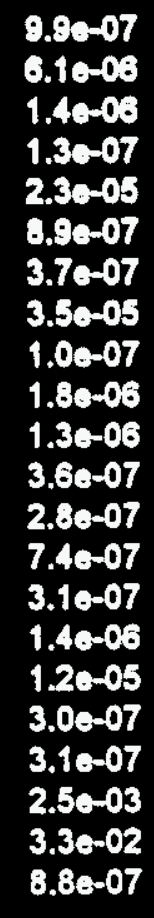 & $20 \%$ \\
\hline
\end{tabular}

$12.50 \%$ Distance correction factor [100\% meane no correction] 9.00\% Atbanution (Sample tubo)

$0.00 \%$ Adenuntion (Etricid)

Enowe and MOA reported at 2 signa

$3-20-95$

Dr. E. Frad Rlod 


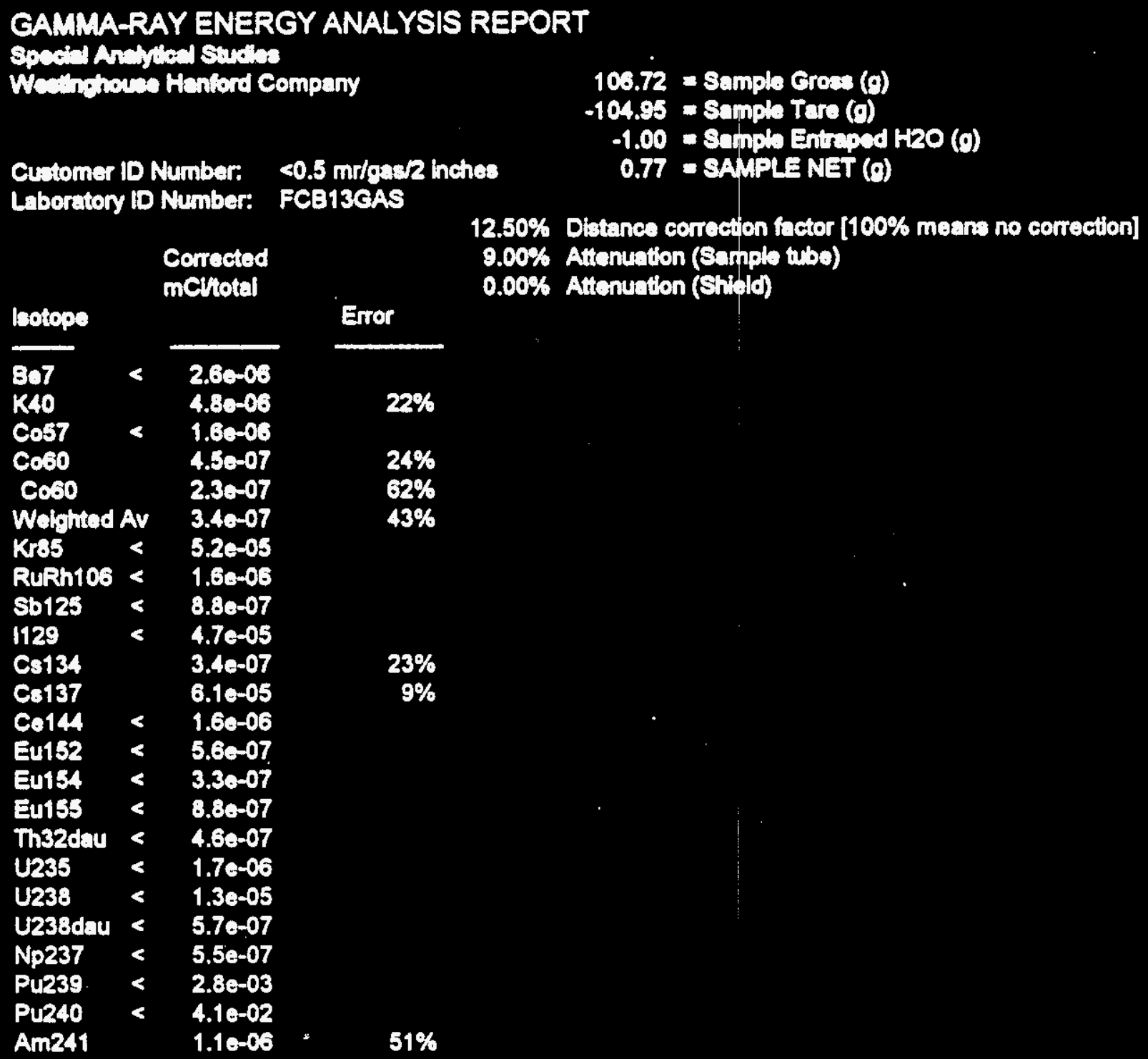

Errose and MDA reported at 2 sioma

Dr. E. Frod Rade 


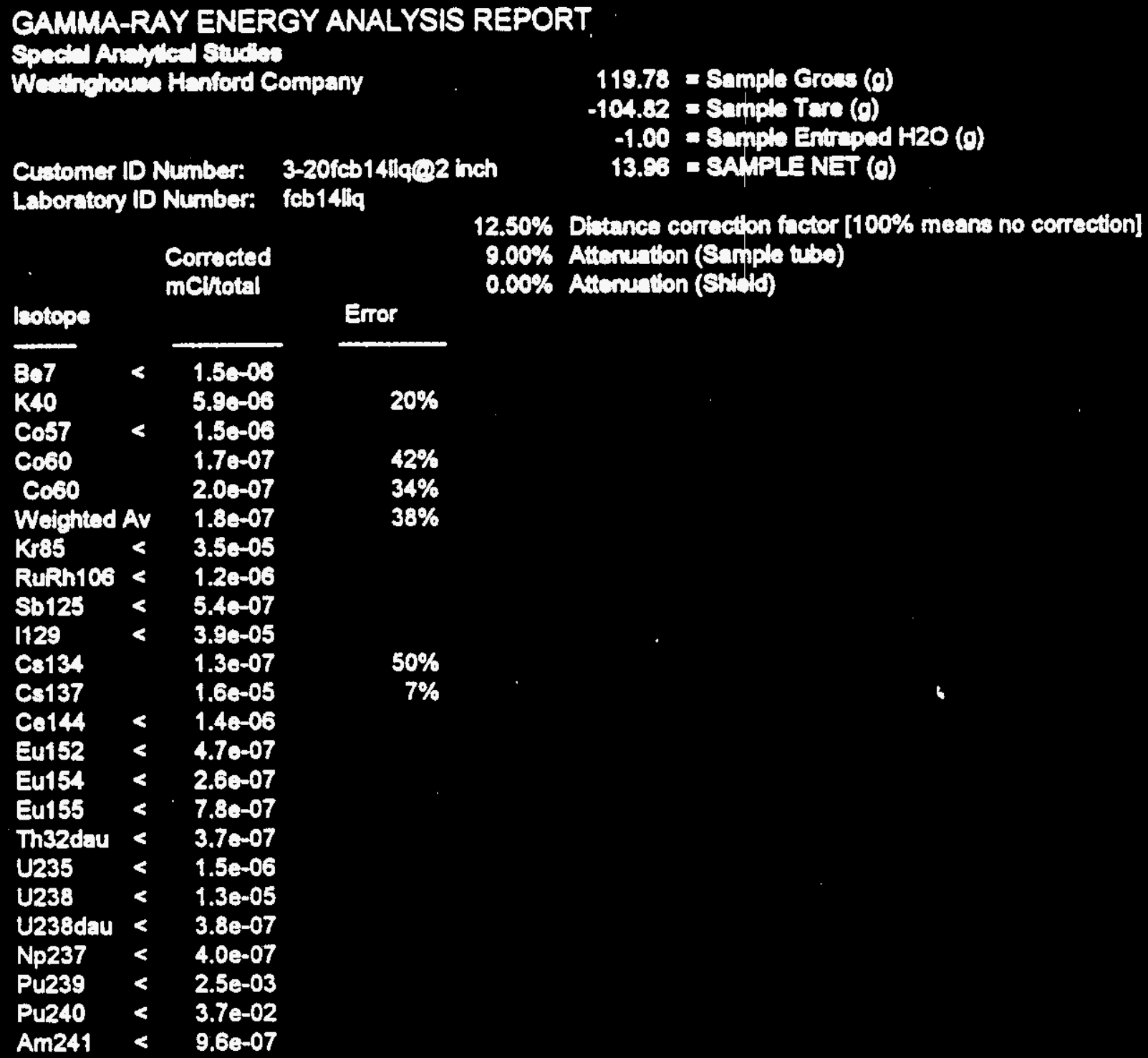

Errore and MOA reported at 2 sigma

$3-20-95$

Dr. EFred Rioded 


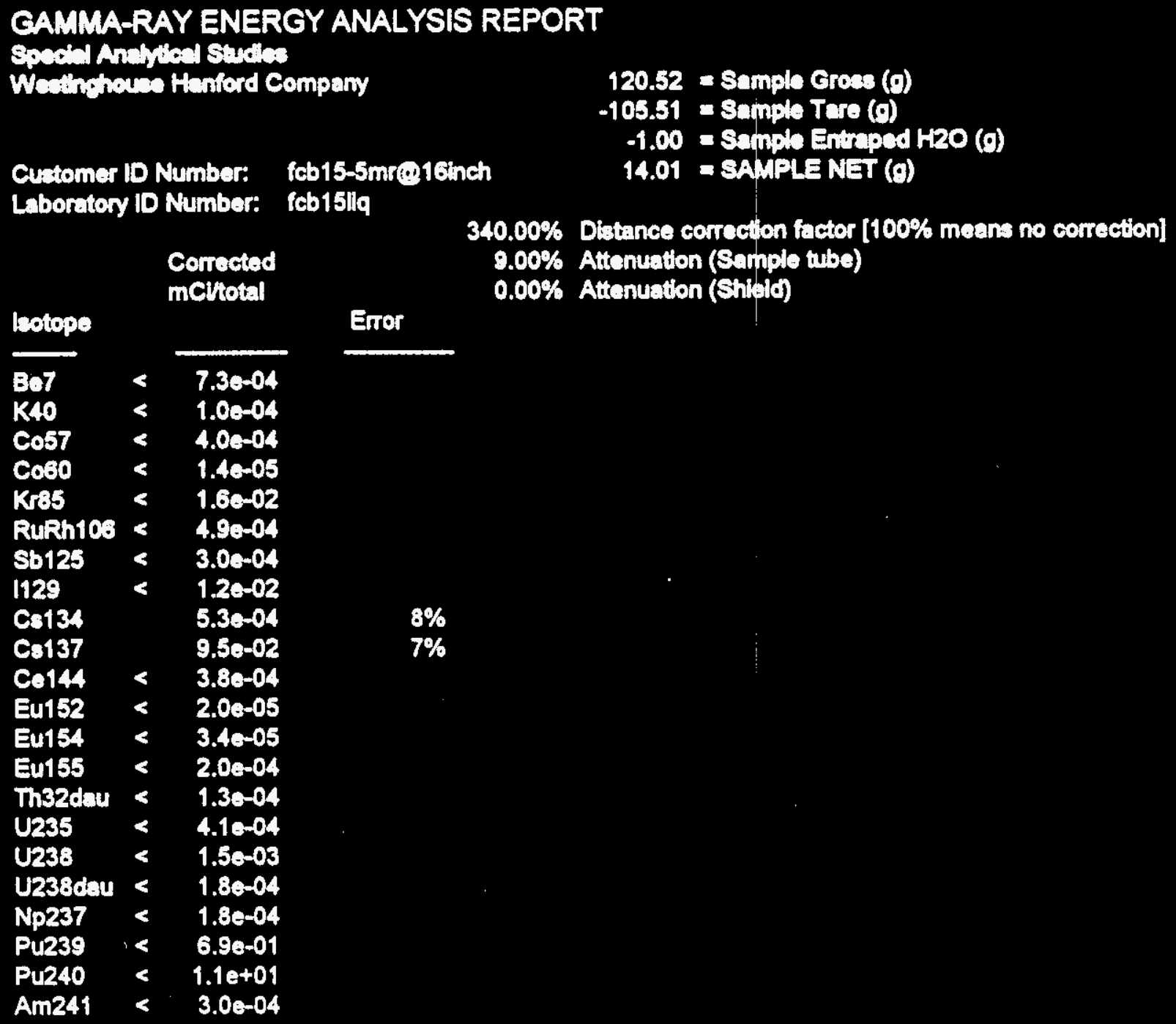

Errore and MDA reported at 2 sigma

3-20-95

Dr. E. Frodradal 


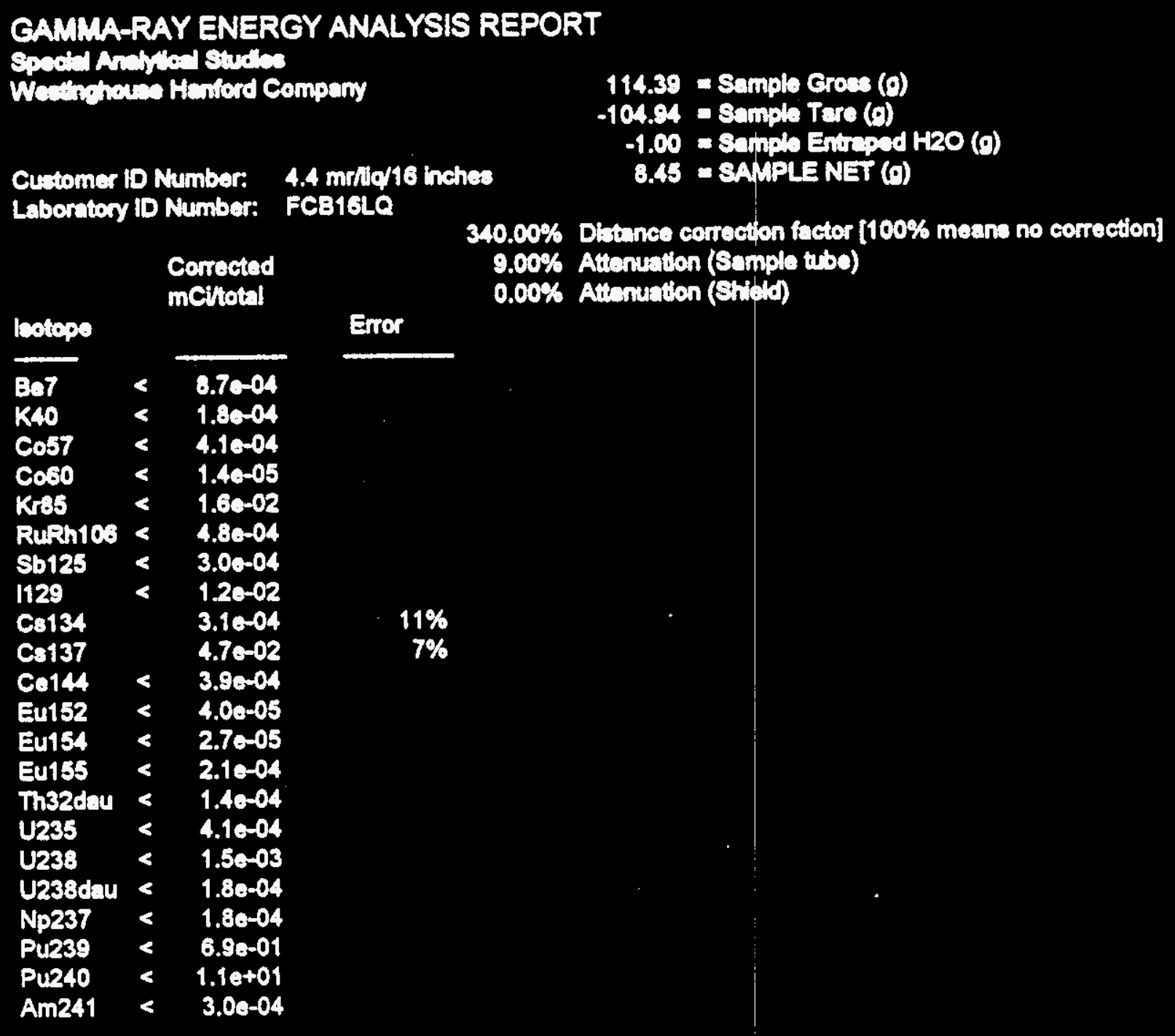

Erros and MDA reported at 2 sions

$3-21 \cdot 96$

Gordon Elomet: 
GAMMA-RAY ENERGY ANALYSIS REPORT

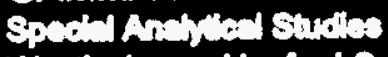

Womithate Hanford Company

Cutomer ID Number: $\quad 1.1 \mathrm{mr} / \mathrm{li} / \mathrm{s}$ inches

Laboratery ID Number: FCB17LQ

\begin{tabular}{|c|c|c|c|}
\hline lsetopo & & $\begin{array}{l}\text { orrected } \\
\text { Cilotal }\end{array}$ & Error \\
\hline $\begin{array}{l}\text { Bat } \\
\text { K40 } \\
\text { Cos7 } \\
\text { Coso } \\
\text { Krss } \\
\text { RuRh10s } \\
\text { Sb125 } \\
\text { I129 } \\
\text { Cs134 } \\
\text { Cs137 } \\
\text { Co144 } \\
\text { Eu152 } \\
\text { Eu154 } \\
\text { Eu155 } \\
\text { Th32du } \\
\text { U235 } \\
\text { U238 } \\
\text { U238dau } \\
\text { Np237 } \\
\text { Pu239 } \\
\text { Pu240 } \\
\text { Am241 }\end{array}$ & $\begin{array}{l}< \\
< \\
< \\
<\end{array}$ & $\begin{array}{l}2.8 e-04 \\
4.7 e-05 \\
1.3 e-04 \\
4.5 e-06 \\
4.9 e-03 \\
1.6 e-04 \\
9.7 e-05 \\
3.9 e-03 \\
1.9 e-04 \\
3.4 e-02 \\
1.2 e-04 \\
7.8 e-06 \\
1.1 e-05 \\
6.5 e-05 \\
4.5 e-05 \\
1.3 e-04 \\
4.3 e-04 \\
5.9 e-05 \\
5.7 e-05 \\
2.2 e-01 \\
3.4 e+00 \\
9.5 e-05\end{array}$ & $8 \%$ \\
\hline
\end{tabular}

Erros and MDA reported at 2 adoma

\section{$3-21-98$}

$$
\begin{aligned}
110.80 & =\text { Sampla Groes }(0) \\
-105.02 & =\text { Sample Tare }(0) \\
-1.00 & =\text { SAmpla Entraped H2O (o) } \\
3.88 & =\text { SAMPLE NET }(\sigma)
\end{aligned}
$$

$100.00 \%$ Distance correction factor [100\% means no correction] $9.00 \%$ Attonuation (Sample tube)

$0.00 \%$ Attenuation (Bhisio)

Gordin Brmat 


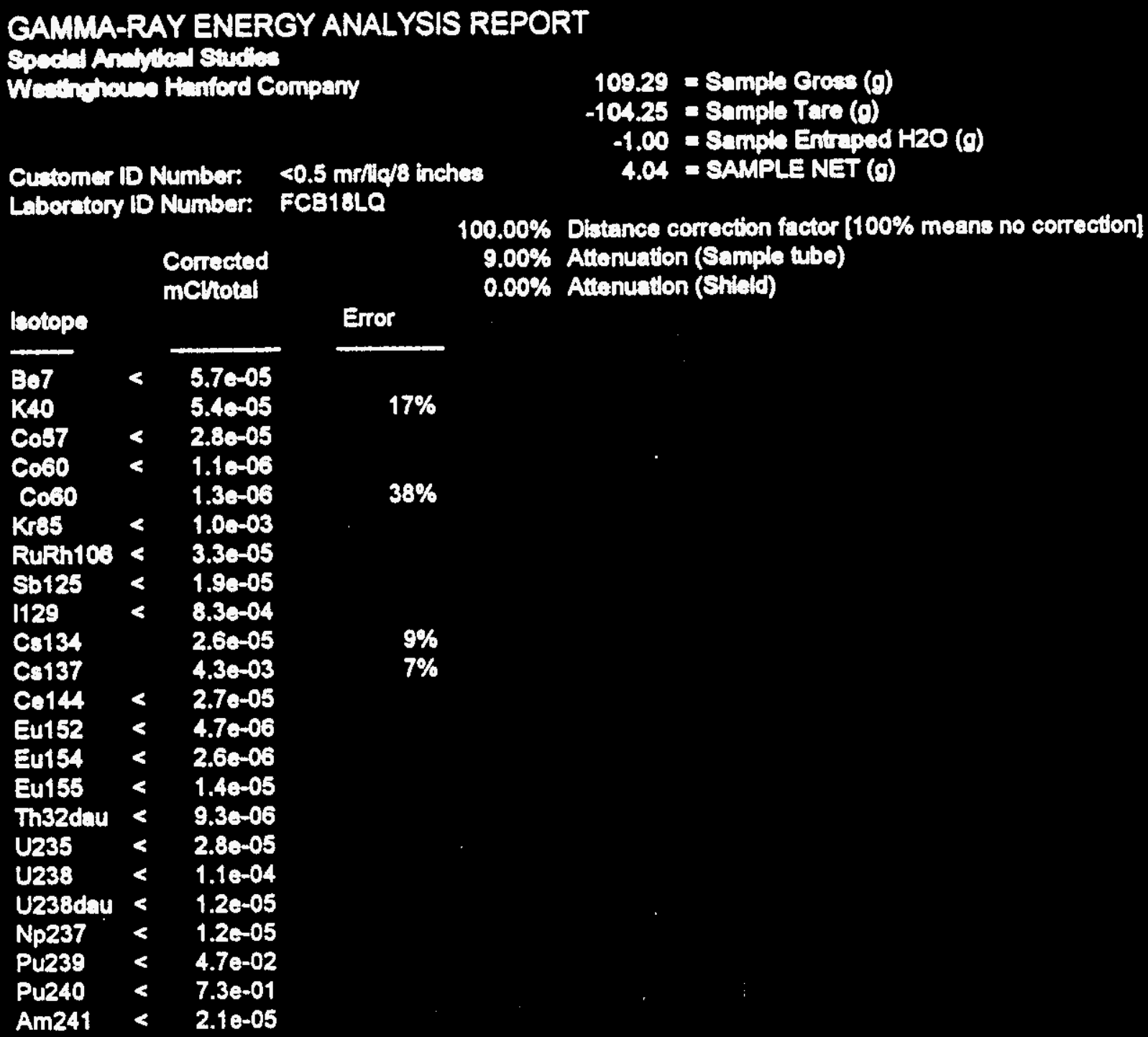

Erren and MDA reported of 2 sioma

$3-21-95$

Corden Elemot 
GAMMA-RAY ENERGY ANALYSIS REPORT

Spacel Anobleol suckes

Wentritious Hentord Company

115.15 - Sample Grose (o)

-104.93 - Sample Tare (0)

-1.00 a Sample Entraped H2O (0)

Customer ID Number: 12 mrill/16 inches

9.22 - SAMPLE NET (o)

Laberatory ID Number: FCB1OLQ

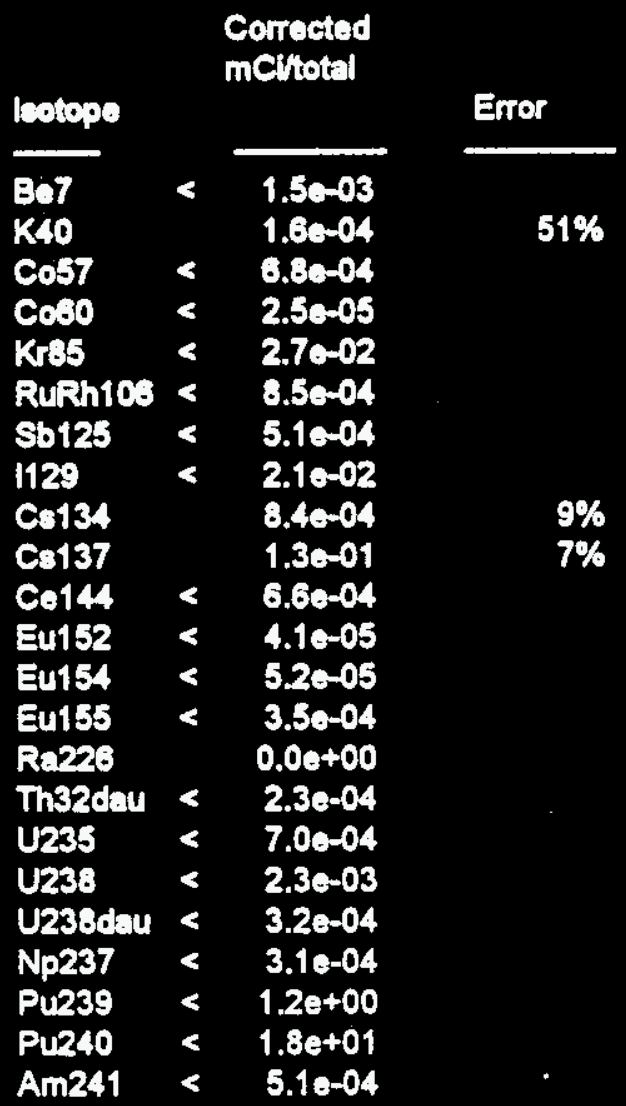

$340.00 \%$ Distance correction factor [ $100 \%$ means no correction]

$\mathbf{9 . 0 0 \%}$ Attenuation (Sample tubo)

$0.00 \%$ Attenuation (Ehield)

$7 \%$

Errone and MDA reported at 2 soms

Gordon Elempit 
WHC-SD-SNF-DP-003; Rev. 0

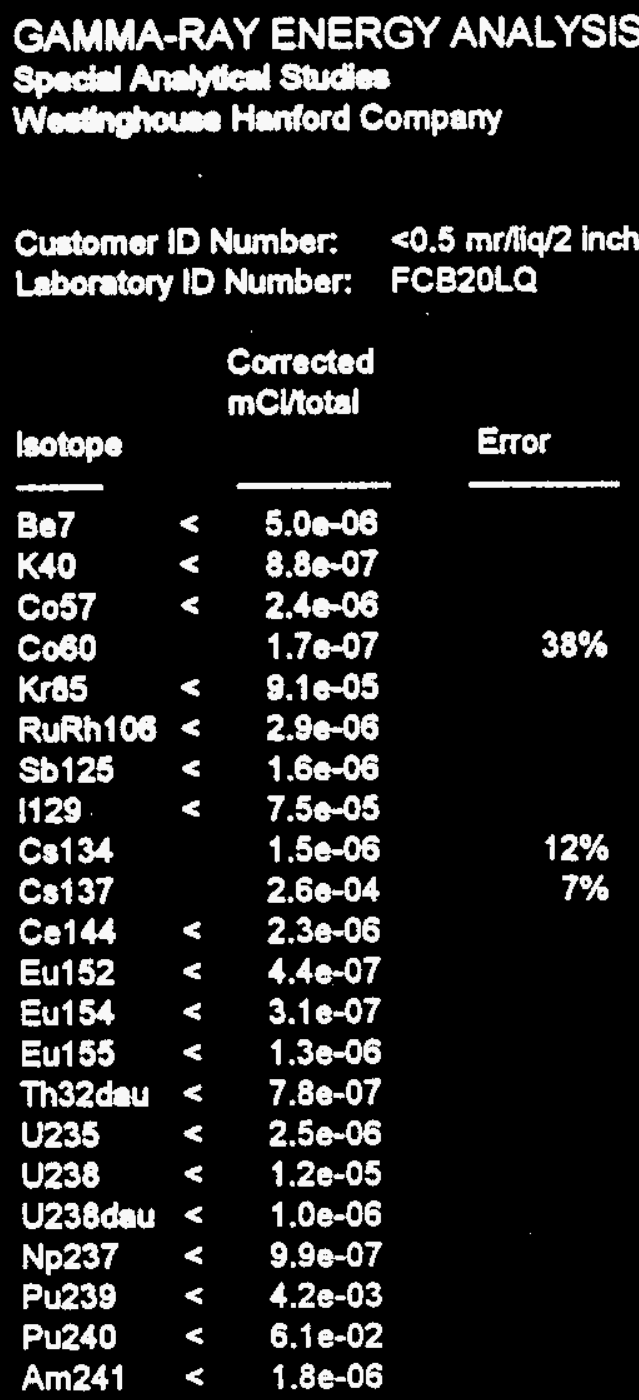

$12.50 \%$ Distance correction factor [100\% means no correction] $9.00 \%$ Attenuation (Sample tube)

$0.00 \%$ Attenuation (Shidd)

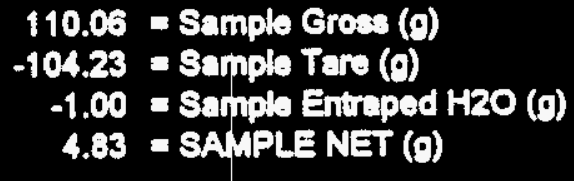


CAMMARAY ENERGY ANALYSIS REPORT

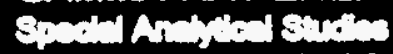

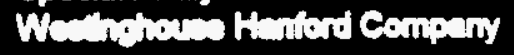

113.88 = Sampla Cross (o)

-107.92 = Bamplo Two (o)

$-1.00=8$ anpib Entraped H2O (o)

Cuntemar iD Number: fob21int at Einch

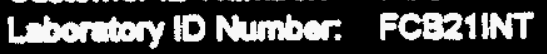

4.78 - SAMPLENET (o)

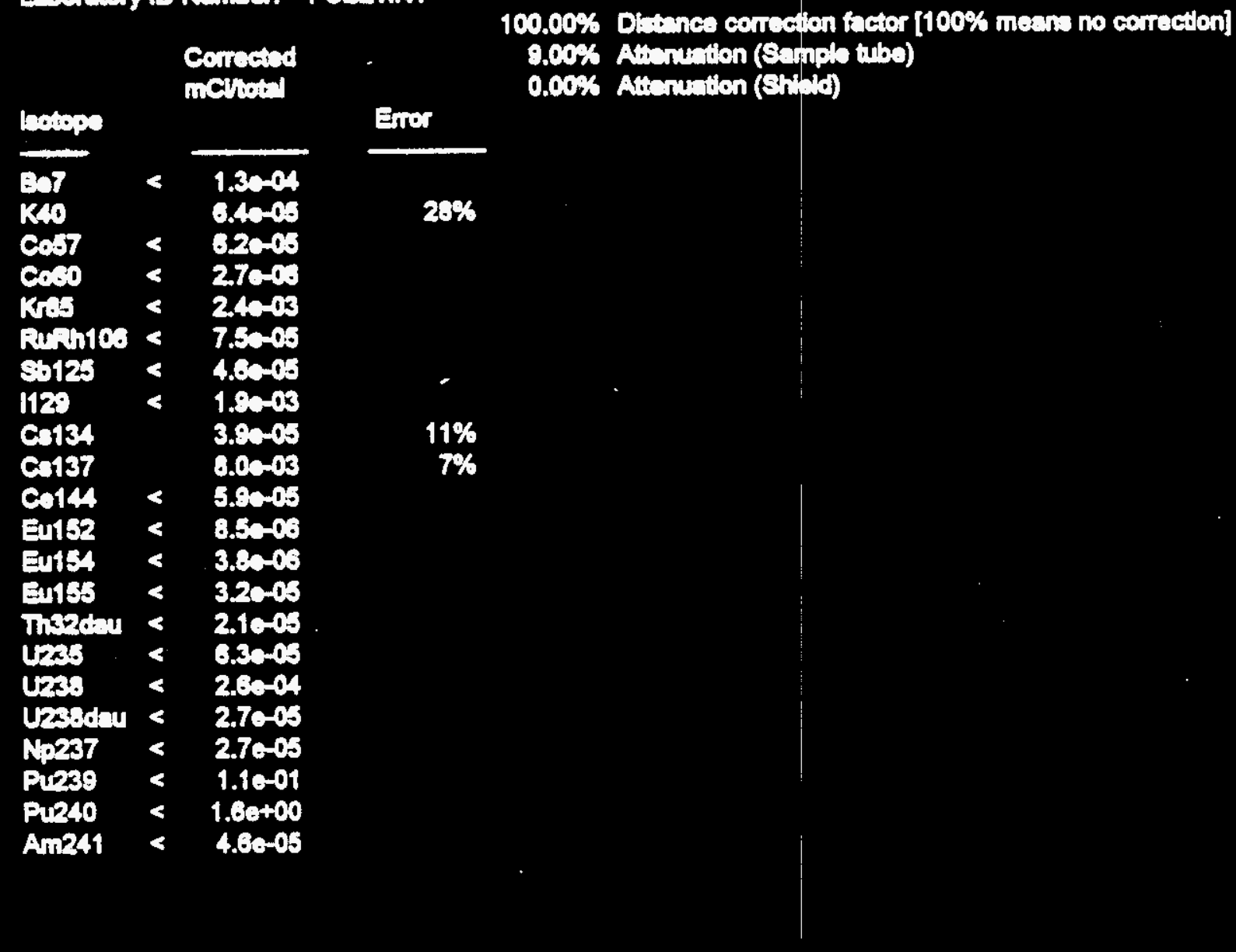

Enow and Mol reportad at 2 ciona

$-3-24.95$

Dr. El Frod Fined 
CAMMARAY ENERCY ANALYSIS REPORT

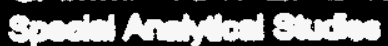

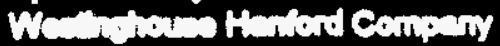

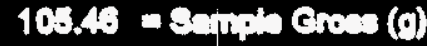

-10201 a suris Tre (a)

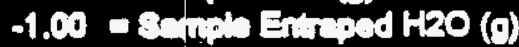

Cunturer if Nunber: forez 324 at 2 inch 1.58 - ANMTLENET (o)

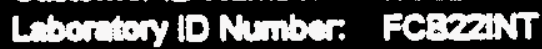

\begin{tabular}{|c|c|c|c|}
\hline besist & & onced & Ener \\
\hline 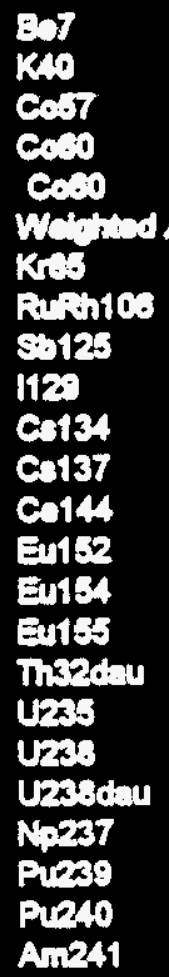 & $\begin{array}{l}< \\
< \\
< \\
< \\
< \\
< \\
< \\
< \\
< \\
< \\
<\end{array}$ & 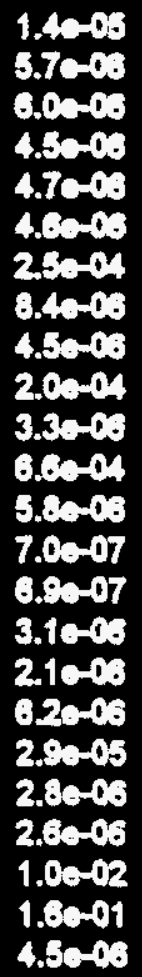 & $\begin{array}{c}30 \% \\
13 \% \\
13 \% \\
13 \% \\
-\end{array}$ \\
\hline
\end{tabular}

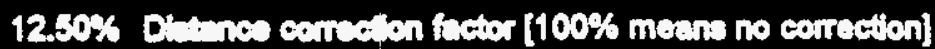

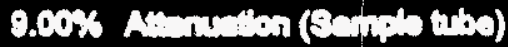

$0.00 \%$ Amorision (Eximg)

$30 \%$

$13 \%$

$13 \%$

$3 \%$

$7 \%$

Erose and MOA repotid at 2 biona $3-2489$

D. E. Frodrion 
WHC-SD-SNF-DP-003, Rev. 0

CAMMARAY ENERGY ANALYSIS REPORT

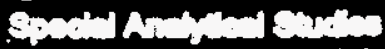

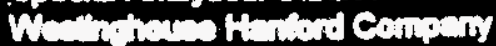

122.78 a sanpio Cran (o)

-108.63 a Sanil Tore (a)

-1.00 = Samph Entrapod H2O (0)

Cuntemer ID Number. 1 mrits in.

16.13 - SAMPLENET (o)

Levertery ID Number: FCezale

\begin{tabular}{|c|c|c|c|}
\hline مקنيف:| & & $\begin{array}{l}\text { orreoted } \\
\text { cellots }\end{array}$ & Error \\
\hline 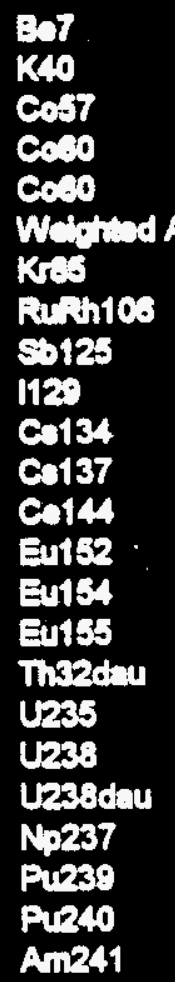 & $\begin{array}{l}< \\
< \\
< \\
< \\
< \\
< \\
< \\
< \\
<\end{array}$ & $\begin{array}{l}8.90-04 \\
7.50-06 \\
420-04 \\
1.40-04 \\
1.30-04 \\
1.30-04 \\
1.60-02 \\
5.60-04 \\
3.00-04 \\
1.30-02 \\
1.20-04 \\
1.60-02 \\
4.00-04 \\
3.70-06 \\
8.50-04 \\
2.10-04 \\
1.40-04 \\
4.20-04 \\
4.20-03 \\
1.80-04 \\
1.80-04 \\
7.20-01 \\
1.10+01 \\
3.10-04\end{array}$ & $\begin{array}{l}23 \% \\
21 \% \\
25 \% \\
-\end{array}$ \\
\hline
\end{tabular}

$100.00 \%$ Dithnce cerrestion factor [100\% mean no correction] $0.00 \%$ Aknim bon (Bampla tobo)

$0.00 \%$ Amonimion (Etimis)

$23 \%$

Tr\%

$25 \%$

$25 \%$

$7 \%$

Erew and MoA reportad at 2 birma

$3-24 \times 9$

Ed Sm 
CAMMA-RAY ENERGY ANALYSIS REPORT

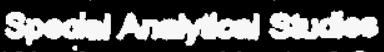

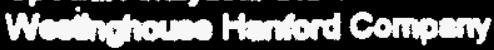

122.70 - Semple Grom (o)

-106.63 a Smpis Tera (a)

-1.00 a Sompin Entrimpand HzO (O)

Cutoner lo Number: 1 morats in.

Lboritery ID Number: FCEzzLAA

18.13 - SAMPLENGT (0)

\begin{tabular}{|c|c|c|c|}
\hline botopes & & $\begin{array}{l}\text { Corroted } \\
\text { nellot:l }\end{array}$ & Entor \\
\hline 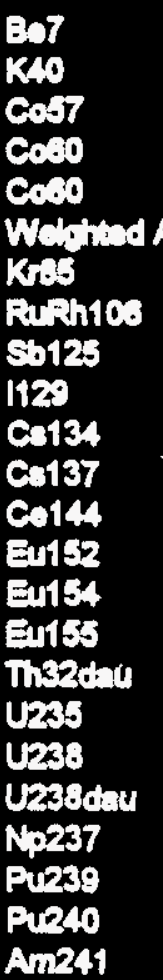 & $\begin{array}{l}l \\
< \\
< \\
< \\
< \\
< \\
< \\
< \\
< \\
<\end{array}$ & $\begin{array}{l}2.60-04 \\
3.00-05 \\
1.20-04 \\
1.10-04 \\
1.10-04 \\
1.10-04 \\
4.70-03 \\
1.60-04 \\
9.20-05 \\
3.80-03 \\
8.10-05 \\
1.60-02 \\
1.20-04 \\
1.1005 \\
8.90-00 \\
6.30-05 \\
4.20-05 \\
1.20-04 \\
6.70-04 \\
5.60-05 \\
5.40-05 \\
2.10-01 \\
3.20+00 \\
9.10-05\end{array}$ & $\begin{array}{c}11 \% \\
11 \% \\
11 \% \\
-\end{array}$ \\
\hline
\end{tabular}

$100.00 \%$ Distance correction factor [100\% means no correction] 8.00\% Ationution (Bemple trbo)

$0.00 \%$ Atmustion (Shides)

Enow and MaA repotad at 2 dorno

$3-24-98$

Ed EलT5 
CAMMARAY ENERGY ANALYSIS REPORT

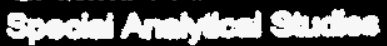

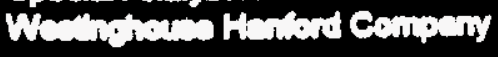

122.76 - Bunpis crom (a)

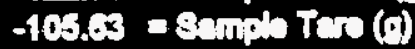

$-1.00=8$ mpol Ethad H20 (0)

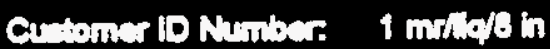

Leboratery lo Number: FCEzales

\begin{tabular}{|c|c|c|c|}
\hline botepe & & oncted & Entor \\
\hline 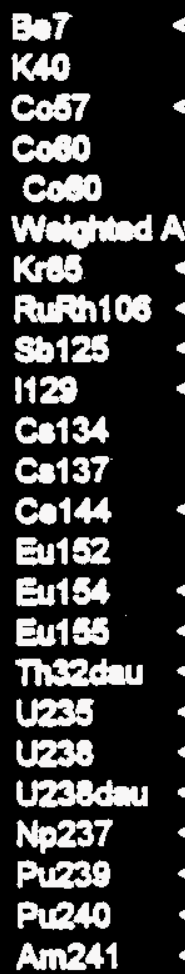 & $\begin{array}{l}< \\
< \\
< \\
< \\
< \\
< \\
<\end{array}$ & $\begin{array}{l}2.70-04 \\
4.70-05 \\
1.20-04 \\
1.10-04 \\
1.10-04 \\
1.10-04 \\
4.00-03 \\
1.60-04 \\
9.30-05 \\
3.70-03 \\
9.8005 \\
1.70-02 \\
1.20-04 \\
5.20-08 \\
1.20-05 \\
9.20-05 \\
4.20-05 \\
1.20-04 \\
7.50-04 \\
5.60-05 \\
5.30-05 \\
2.10-01 \\
3.20+00 \\
9.3005\end{array}$ & $\begin{array}{c}40 \% \\
11 \% \\
11 \% \\
11 \% \\
-\end{array}$ \\
\hline
\end{tabular}

$100.00 \%$ Dimance cerreation facter [100\% means no correction] 0.00\% Albmution (Eampla trbo)

$0.00 \%$ Atmention (Ehiold)

$1 \%$

$\%$

$2 \%$

Evore end MaA roported et 2 ciona

Ed 8cro 
GAMMARAY ENERGY ANALYSIS REPORT

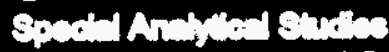

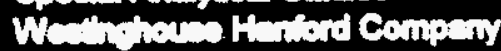

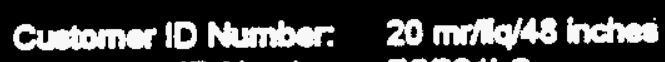

Lebostony ID Number. FCe24he

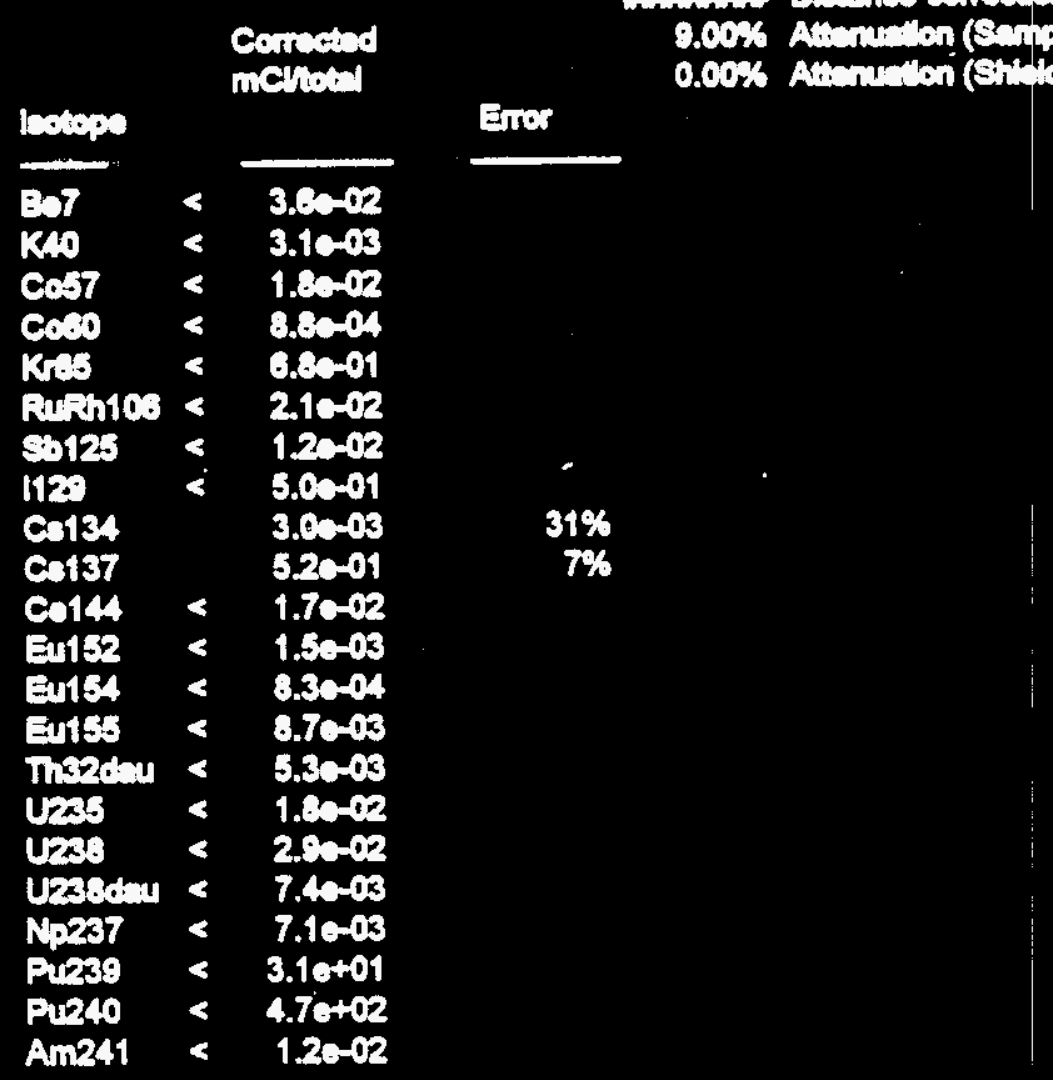

Correntad

bosise

Bi

Kin

Ru:Th103

50125

Cot37

Co144

En15e

Eu15.

Ents:

Throtion

res

Lanas

No237

An241
Kras
$121.01=$ Sompib Goos $(0)$

-108.44 - Sunim TaO (a)

-1.00 - Empin Entrad HeO (o)

14.57 a EAMUL NaT (a)

Enose and MDA reported at 2 ingha

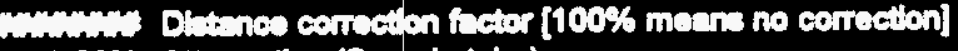

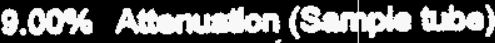

$0.00 \%$ Andurison (Enimid) 
CAMMA-RAY ENERGY ANALYSIS REPORT

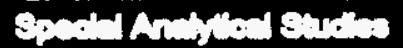

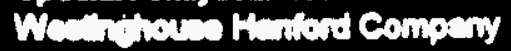

Cutomer ID Number: 1.5 mrild/16 inches Leboratery ID Number: FCE2sLa

\begin{tabular}{|c|c|c|c|}
\hline 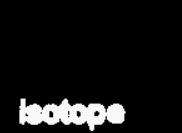 & & $\begin{array}{l}\text { Correotod } \\
\text { mellibtal }\end{array}$ & \\
\hline 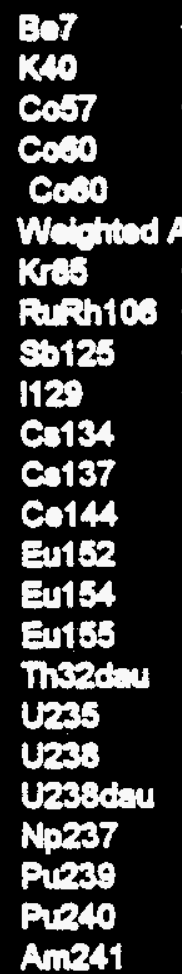 & $\begin{array}{l}< \\
< \\
\text { Av } \\
< \\
< \\
< \\
< \\
< \\
< \\
< \\
< \\
< \\
< \\
< \\
< \\
< \\
< \\
< \\
<\end{array}$ & $\begin{array}{l}6.60-04 \\
1.60-04 \\
3.10-04 \\
9.00-04 \\
1.00-04 \\
9.70-06 \\
1.30-02 \\
3.90-04 \\
2.20-04 \\
0.70-03 \\
1.10-04 \\
2.00-02 \\
3.00-04 \\
1.60-0 . \\
2.60-06 \\
1.60-04 \\
1.00-04 \\
3.10-04 \\
1.60-03 \\
1.40-04 \\
1.30-04 \\
5.40-01 \\
8.10+00 \\
2.30-04\end{array}$ & $\begin{array}{l}53 \% \\
21 \% \\
20 \% \\
21 \% \\
\end{array}$ \\
\hline
\end{tabular}

$$
\begin{aligned}
& 120.40 \text { - Sanplo Crose (o) } \\
& -106.43 \text { - Sunpis Tre (a) } \\
& -1.00 \text { a Sathin Entrad H2O (0) } \\
& 14.03 \text { - sAMPLE NET (o) }
\end{aligned}
$$

$340.00 \%$ Dithnes correction fadter [100\% means no correction] 8.00\% Amanmion (Bamplo trbo)

$0.00 \%$ Ansurilion (Erital)

Esowe and MOA repoted at 2 ciona 
GAMMA-RAY ENERGY ANALYSIS REPORT

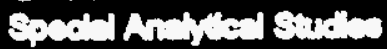

Wementouns Hentord Compary

$$
\begin{aligned}
& 122.51 \text { = Sample Crose (a) } \\
& -107.60 \text { - Sample Tor (O) } \\
& -1.00 \text { - Sanplo Entroed HzO (o) } \\
& 13.91 \text { - SAMPLE NET (o) }
\end{aligned}
$$

Leberitery lo Number: FCS2oLe

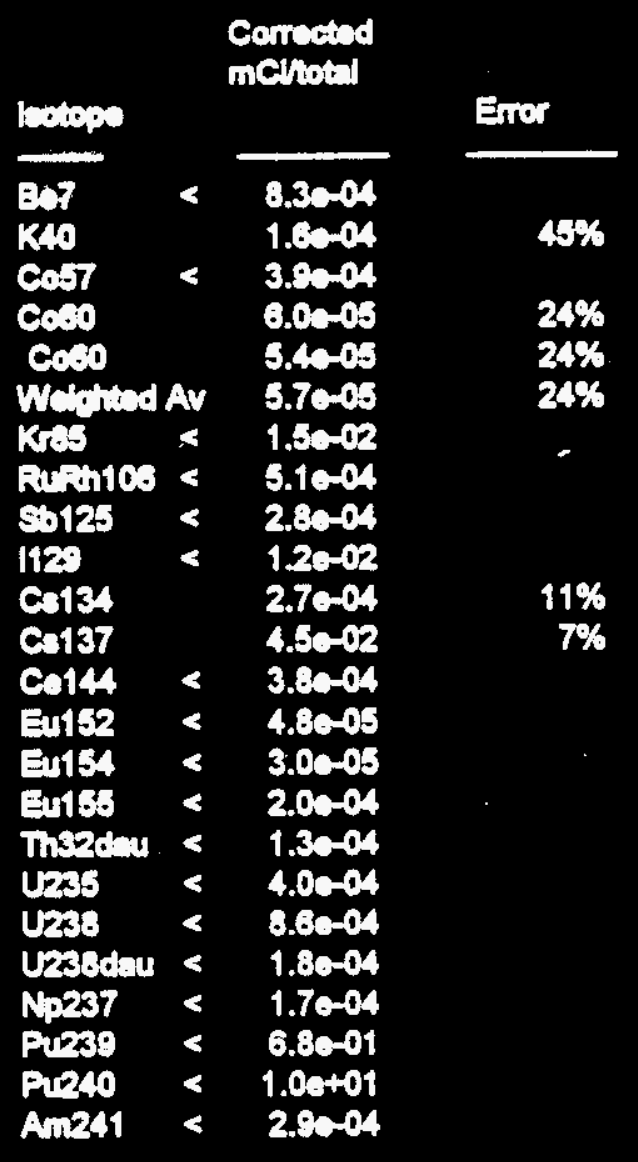

340.00\% Dibince correction factor [100\% mean no correction] 9.00\% Ationution (Eamplo trbo) $0.00 \%$ Atwontion (Ehivid)

Eirse and MaA repetid at 2 ciona $3-24 \times 8$

Cordon Ermett 
WHC-SD-SNF-DP-003, Rev. 0

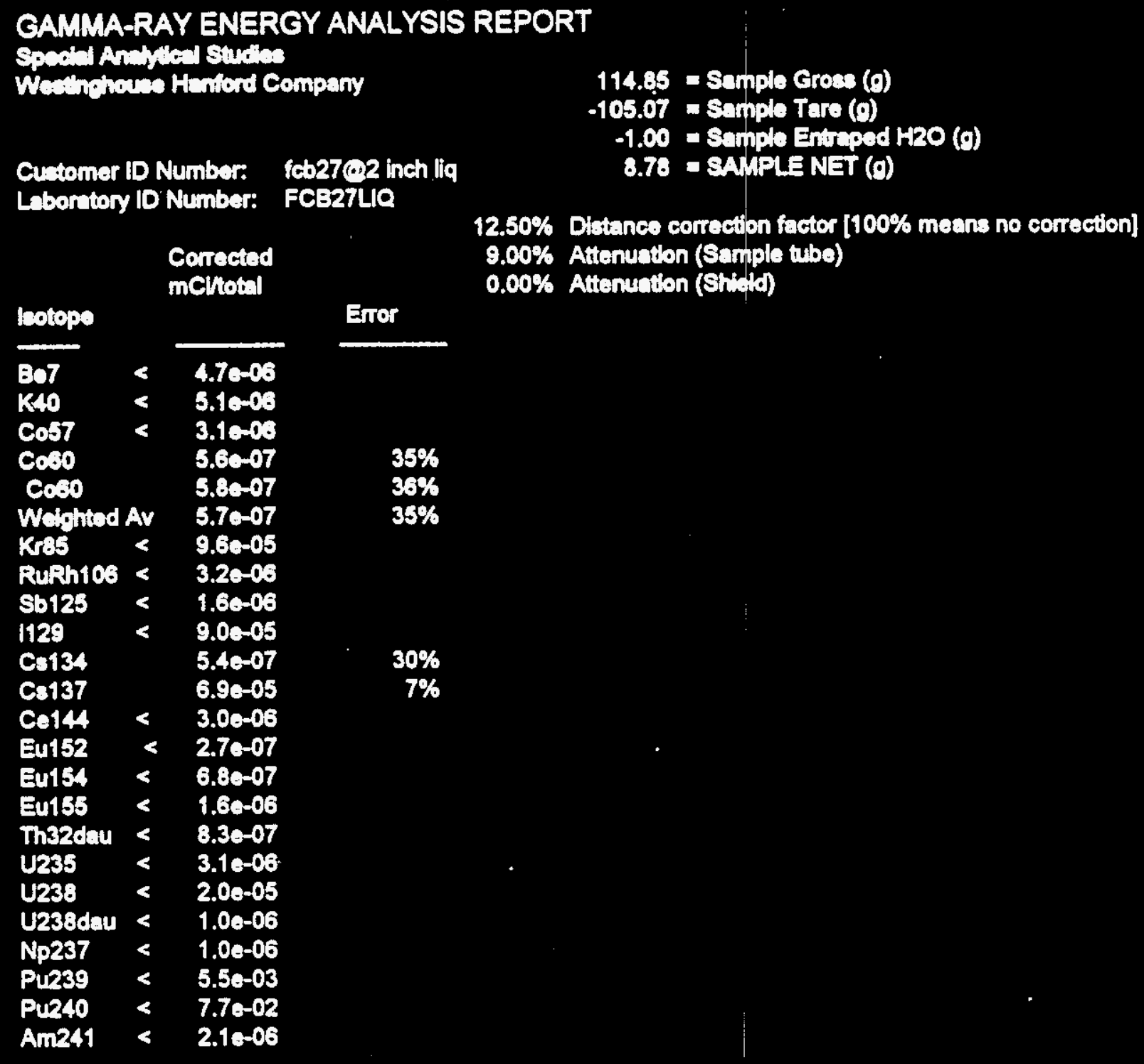

Erose and MDA reportod at 2 cioma

$3-25095$

Dr. E. Fred Riodel 
WHC-SD-SNF-DP-003, Rev. 0

CAMMA-RAY ENERGY ANALYSIS REPORT

Spacth Andived suctes

Wentionote Hertiord Company

$113.90=$ Sample Grows (o)

$-104.49=$ Sample Tare (o)

-1.00 - Sanple Entraped H2O (o)

Customer ID Number: feb28932in <.5mr liq

8.41 - SAMPLE NET (o)

Laboratory ID Number: FCB28LJa

\begin{tabular}{|c|c|c|c|}
\hline botope & & $\begin{array}{l}\text { Conrocted } \\
\text { nCltotal }\end{array}$ & Error \\
\hline $\begin{array}{l}\text { Bo7 } \\
\text { K40 } \\
\text { Cos7 } \\
\text { Co60 } \\
\text { Co60 } \\
\text { Kros } \\
\text { RuRh10s } \\
\text { Sb125 } \\
1129 \\
\text { Cs134 } \\
\text { Cs137 } \\
\text { Ce144 } \\
\text { Eu152 } \\
\text { Eu154 } \\
\text { Eu155 } \\
\text { Th32dau } \\
\text { U235 } \\
\text { U238 } \\
\text { U238dau } \\
\text { Np237 } \\
\text { Pu239 } \\
\text { Pu240 } \\
\text { Am241 }\end{array}$ & $\begin{array}{l}< \\
< \\
< \\
< \\
< \\
< \\
< \\
< \\
< \\
< \\
< \\
<\end{array}$ & $\begin{array}{l}7.56-08 \\
8.5 e-06 \\
2.86-06 \\
2.6 e-07 \\
2.20-07 \\
8.00-05 \\
2.7 e-06 \\
1.3 e-06 \\
8.26-05 \\
2.26-07 \\
4.7 e-05 \\
2.7 e-06 \\
9.1 e-07 \\
6.8 e-07 \\
1.5 e-06 \\
8.16-07 \\
2.9 e-06 \\
1.8 e-05 \\
8.8 e-07 \\
8.6 e-07 \\
4.9 e-03 \\
7.6 e-02 \\
2.0 e-06\end{array}$ & $53 \%$ \\
\hline
\end{tabular}

$12.50 \%$ Distance correction factor [100\% means no correction] $9.00 \%$ Attenuation (Sample tube)

$0.00 \%$ Attenuation (Shidd)

$7 \%$

Errore and MDA reported at 2 ciame

$3-26-95$

Dr. Es Fred Fidd 
CAMMA-RAY ENERGY ANALYSIS REPORT

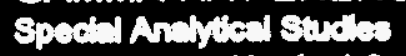

Weitribue Honford Company

119.79 - Sample Groes (g)

$-103.53=$ Sample Tare $(0)$

-1.00 - Sompla Entraped H2O (o)

Customer ID Number: feb2:ing:0 in, $7 \mathrm{mr}$ 16.26 - SAMPLENET $(0)$

Laboratory ID Number: FCB2sLIa

\begin{tabular}{|c|c|c|c|}
\hline leotopese & & $\begin{array}{l}\text { Corrected } \\
\text { methotal }\end{array}$ & Error \\
\hline $\begin{array}{l}\text { Bo7 } \\
\text { K40 } \\
\text { Co57 } \\
\text { Co60 } \\
\text { Coso } \\
\text { Weighted } \\
\text { Kr85 } \\
\text { RuRh10s } \\
\text { Sb125 } \\
\text { I129 } \\
\text { Cs134 } \\
\text { Cs137 } \\
\text { Ce144 } \\
\text { Eu152 } \\
\text { Eu154 } \\
\text { Eu155 } \\
\text { Th32dau } \\
\text { U235 } \\
\text { U238 } \\
\text { U238dau } \\
\text { Np237 } \\
\text { Pu239 } \\
\text { Pu240 } \\
\text { Am241 }\end{array}$ & $\begin{array}{l}< \\
< \\
< \\
< \\
< \\
< \\
< \\
< \\
< \\
< \\
< \\
<\end{array}$ & $\begin{array}{l}1.3 e-04 \\
4.6 e-05 \\
6.1 e-05 \\
4.8 e-05 \\
5.0 e-05 \\
4.9 e-05 \\
2.4 e-03 \\
7.3 e-05 \\
4.5 e-05 \\
1.86-03 \\
3.9 e-05 \\
7.5 e-03 \\
5.8 e-05 \\
5.6 e-05 \\
4.5 e-06 \\
3.0 e-05 \\
2.1 e-05 \\
6.2 e-05 \\
3.4 e-04 \\
2.7 e-05 \\
2.7 e-05 \\
1.0 e-01 \\
1.6 e+00 \\
4.5 e-05\end{array}$ & $\begin{array}{l}36 \% \\
12 \% \\
11 \% \\
11 \%\end{array}$ \\
\hline
\end{tabular}

$100.00 \%$ Distance correction factor [ $100 \%$ means no correction] $9.00 \%$ Attenuation (Sample tube)

$0.00 \%$ Attenuation (Shivld)

Erore and MDA reportod at 2 sioma

Dr. E. Fred Alodel

\section{$-3-25-6$}


GAMMA-RAY ENERGY ANALYSIS REPORT

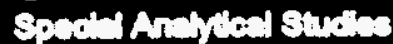

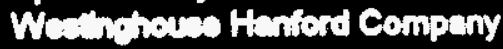

Cutomer ID Number: fob3004ik-40MR Laboratory ID Number: FCB30LIa

\begin{tabular}{|c|c|c|c|}
\hline Eotope & & $\begin{array}{l}\text { Corrected } \\
\text { metrotal }\end{array}$ & Error \\
\hline $\begin{array}{l}\text { Be7 } \\
\text { K40 } \\
\text { Co57 } \\
\text { Cos0 } \\
\text { Kr85 } \\
\text { RuPh108 } \\
\text { Sb125 } \\
\text { I129 } \\
\text { Cs134 } \\
\text { Cs137 } \\
\text { Ce144 } \\
\text { Eu152 } \\
\text { Eu154 } \\
\text { Eu155 } \\
\text { Th32dau } \\
\text { U235 } \\
\text { U238 } \\
\text { U238dau } \\
\text { Np237 } \\
\text { Pu239 } \\
\text { Pu240 } \\
\text { Am241 }\end{array}$ & $\begin{array}{l}< \\
< \\
< \\
< \\
< \\
< \\
< \\
< \\
<\end{array}$ & $\begin{array}{l}9.90-03 \\
1.50-03 \\
4.9 e-03 \\
1.40-04 \\
1.90-01 \\
5.9 e-03 \\
3.40-03 \\
1.5 e-01 \\
3.00-03 \\
6.0 e-01 \\
4.7 e-03 \\
1.10-04 \\
3.7 e-04 \\
2.5 e-03 \\
1.6 e-03 \\
5.0 e-03 \\
1.2 e-02 \\
2.10-03 \\
2.1 e-03 \\
8.4 e+00 \\
1.3 e+02 \\
3.6 e-03\end{array}$ & $\begin{array}{r}11 \% \\
7 \%\end{array}$ \\
\hline
\end{tabular}

121.77 - Sampla Groas (g)

-105.98 a Sample Tare (0)

-1.00 - Sample Entraped H2O (o)

14.81 - SAMPLENET (o)

Hinniniti: Distance correction factor [100\% means no correction]

$9.00 \%$ Attenuation (Sample tubo)

$0.00 \%$ Attenuation (Shivid)

Errose and MDA reportod at 2 sigma

Dr. E. Fred Firot

$3-25-95$ 


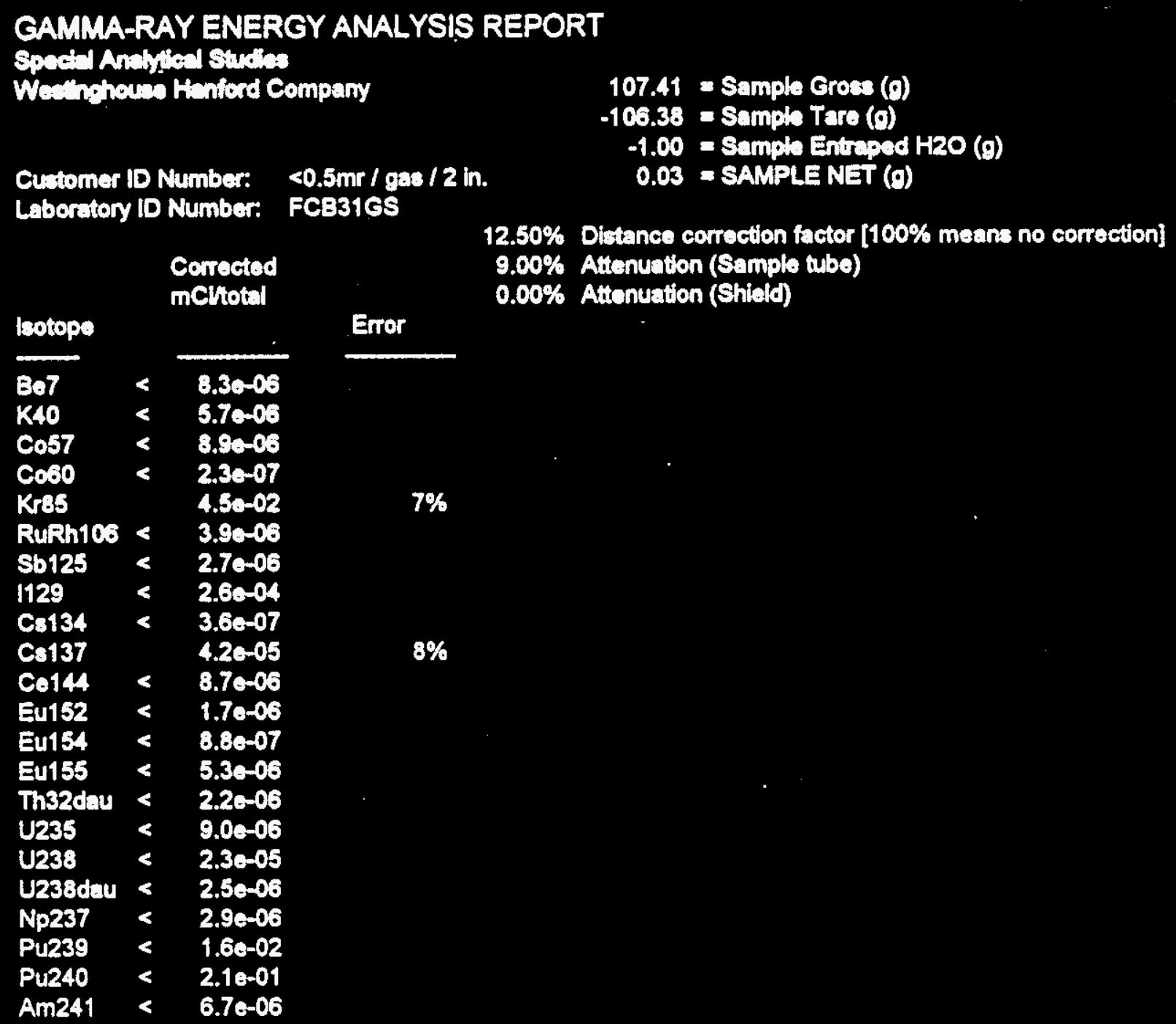

Errors and MDA reported at 2 sigma 


\section{GAMMA-RAY ENERGY ANALYSIS REPORT

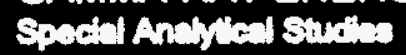 \\ Woulutious Henford Company}

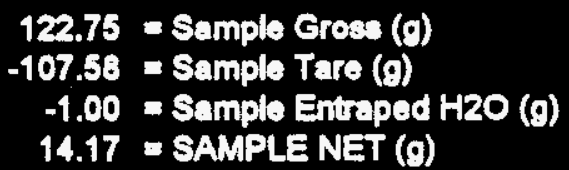

Cuttomer ID Number: $\quad<0.5 \mathrm{mr} / \mathrm{liq} / 2 \mathrm{in}$. Laboratory iD Number: FCB32LQ

\begin{tabular}{|c|c|c|c|}
\hline leotope & & $\begin{array}{l}\text { Corrected } \\
\text { ncinotal }\end{array}$ & Error \\
\hline $\begin{array}{l}\text { Bo7 } \\
\text { K40 } \\
\text { Co57 } \\
\text { Coso } \\
\text { Co60 } \\
\text { Wolghted } \\
\text { Kr85 } \\
\text { RuRh106 } \\
\text { Sb125 } \\
\text { I129 } \\
\text { Co134 } \\
\text { Cs137 } \\
\text { Ce144 } \\
\text { Eu152 } \\
\text { Eu154 } \\
\text { Eu155 } \\
\text { Th32dau } \\
\text { U235 } \\
\text { U238 } \\
\text { U238dau } \\
\text { Np237 } \\
\text { Pu239 } \\
\text { Pu240 } \\
\text { Am241 }\end{array}$ & $\begin{array}{l}< \\
< \\
< \\
< \\
< \\
< \\
< \\
< \\
< \\
< \\
<\end{array}$ & $\begin{array}{l}2.9 e-06 \\
1.5 e-06 \\
1.8 e-06 \\
2.2 e-07 \\
1.6 e-07 \\
1.9 e-07 \\
6.7 e-05 \\
1.9 e-06 \\
9.8 e-07 \\
5.2 e-05 \\
4.1 e-07 \\
8.1 e-05 \\
1.7 e-06 \\
5.1 e-07 \\
2.3 e-07 \\
9.8 e-07 \\
5.0 e-07 \\
1.8 e-06 \\
1.4 e-05 \\
6.2 e-07 \\
5.9 e-07 \\
3.0 e-03 \\
4.5 e-02 \\
1.3 e-06\end{array}$ & $\begin{array}{l}23 \% \\
33 \% \\
44 \% \\
38 \%\end{array}$ \\
\hline
\end{tabular}

$12.50 \%$ Distance correction factor [ $100 \%$ means no correction] 9.00\% Attenuation (Sample tube)

$0.00 \%$ Attenustion (Shieid) 


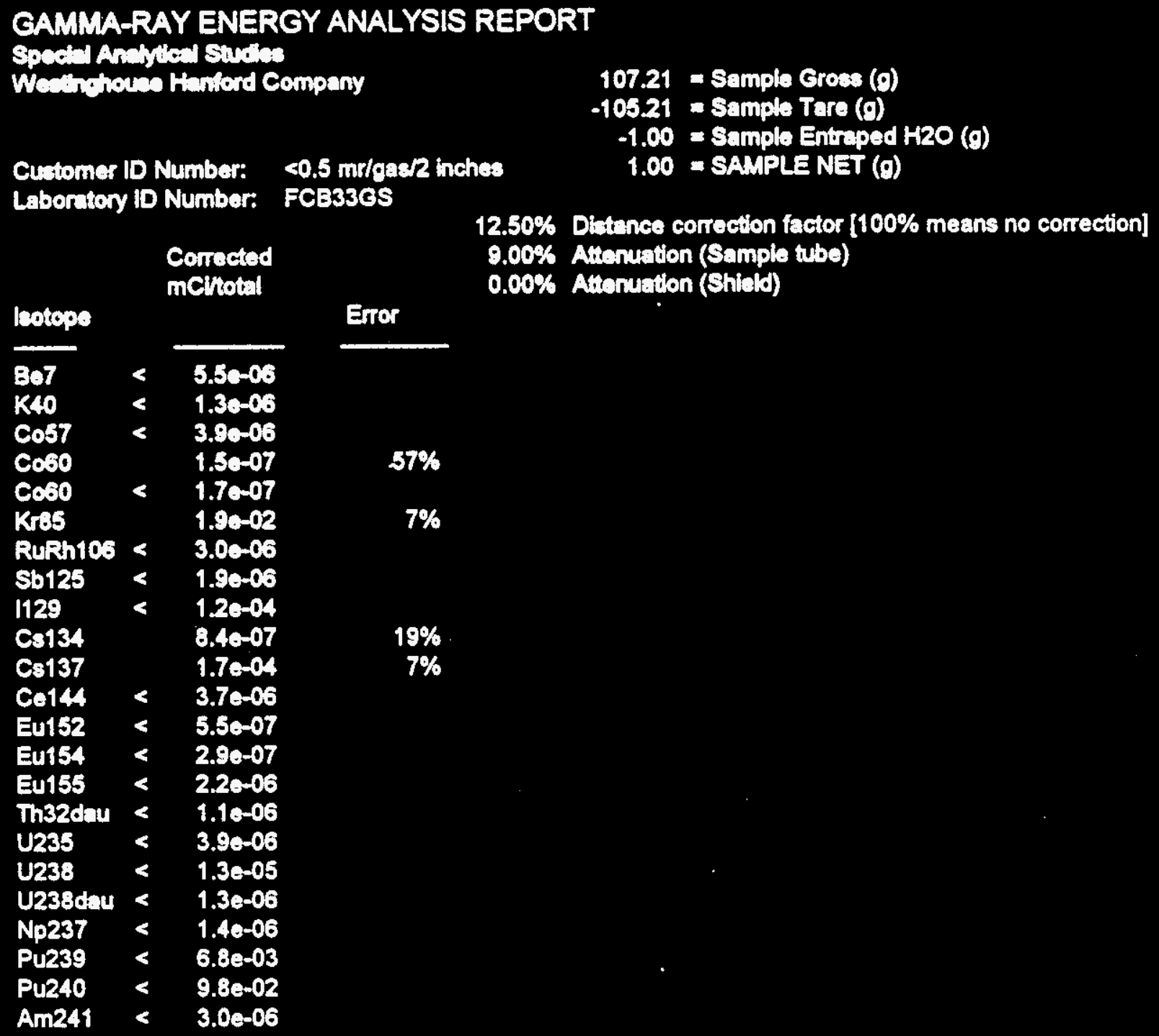

Errore and MDA reported at 2 sigme

$3-25.05$

Gorden Elomer: 
GAMMA-RAY ENERGY ANALYSIS REPORT

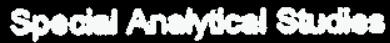

Weatribious Hanferd Company

$$
\begin{aligned}
108.75 & =\text { Sample Crose }(0) \\
-108.59 & =\text { Sample Tare }(0) \\
-1.00 & =\text { Sample Entraped H2O (o) } \\
1.16 & =\text { SAMPLE NET }(0)
\end{aligned}
$$

\begin{tabular}{|c|c|c|c|}
\hline botepe & & $\begin{array}{l}\text { Donected } \\
\text { nertotal }\end{array}$ & Enor \\
\hline $\begin{array}{l}\text { Be7 } \\
\text { K40 } \\
\text { Co57 } \\
\text { Co60 } \\
\text { Co60 } \\
\text { Woighted A } \\
\text { Kr85 } \\
\text { RuRh10s } \\
\text { Sb125 } \\
\text { l129 } \\
\text { Cs134 } \\
\text { Cs137 } \\
\text { Co144 } \\
\text { Eu152 } \\
\text { Eu154 } \\
\text { Eu155 } \\
\text { Th32dau } \\
\text { U235 } \\
\text { U238 } \\
\text { U238dau } \\
\text { Np237 } \\
\text { Pu239 } \\
\text { Pu240 } \\
\text { Am241 }\end{array}$ & $\begin{array}{l}< \\
< \\
\text { Av } \\
< \\
< \\
< \\
<\end{array}$ & $\begin{array}{l}2.1 e-06 \\
4.9 e-06 \\
2.9 e-06 \\
7.4 e-07 \\
4.40-07 \\
5.9 e-07 \\
2.5 e-02 \\
1.1 e-06 \\
7.5 e-07 \\
8.9 e-05 \\
1.2 e-07 \\
7.2 e-06 \\
2.9 e-06 \\
5.6 e-07 \\
2.6 e-07 \\
1.7 e-06 \\
7.2 e-07 \\
2.9 e-06 \\
1.1 e-05 \\
7.6 e-07 \\
9.3 e-07 \\
5.2 e-03 \\
7.0 e-02 \\
2.2 e-06\end{array}$ & $\begin{array}{r}21 \% \\
17 \% \\
39 \% \\
28 \% \\
7 \%\end{array}$ \\
\hline
\end{tabular}

Cutomer ID Number: $<0.5 \mathrm{mr} / \mathrm{gas} / 2 \mathrm{in}$.

Labortory ID Number: FCB34OS

$12.50 \%$ Distance correction factor [100\% means no correction] 9.00\% Attenuation (Sample tube)

$0.00 \%$ Atronuation (Shiclo)

$\%$

Errore and MDA reported at 2 sioma

$3-26.98$

Ed Sew 
GAMMA-RAY ENERGY ANALYSIS REPORT

Specil Anditioal studios

Weathetowe Hantord Company

$$
\begin{aligned}
119.34 & =\text { Sample Gross }(0) \\
-103.44 & =\text { Sample Tare }(0) \\
-1.00 & =\text { SAmple Entraped H2O (o) } \\
14.50 & =\text { SAMPLE NET }(0)
\end{aligned}
$$

\begin{tabular}{|c|c|c|c|}
\hline leotope & & $\begin{array}{l}\text { Corrected } \\
\text { ncthotal }\end{array}$ & Error \\
\hline $\begin{array}{l}\text { Be7 } \\
\text { K10 } \\
\text { Cos7 } \\
\text { Cos0 } \\
\text { Kr85 } \\
\text { RuRh108 } \\
\text { Sb125 } \\
1129 \\
\text { Cs134 } \\
\text { Cs137 } \\
\text { Co144 } \\
\text { Eu152 } \\
\text { Eu154 } \\
\text { Eu155 } \\
\text { Th32dau } \\
\text { U235 } \\
\text { U238 } \\
\text { U238dau } \\
\text { Np237 } \\
\text { Pu239 } \\
\text { Pu240 } \\
\text { Am241 }\end{array}$ & $<$ & $\begin{array}{l}4.5 e-03 \\
1.4 e-03 \\
2.3 e-03 \\
8.3 e-05 \\
8.6 e-02 \\
2.6 e-03 \\
1.6 e-03 \\
6.9 e-02 \\
1.5 e-03 \\
2.7 e-01 \\
2.2 e-03 \\
1.80-04 \\
2.1 e-04 \\
1.20-03 \\
7.3 e-04 \\
2.4 e-03 \\
5.2 e-03 \\
9.8 e-04 \\
9.5 e-04 \\
4.0 e+00 \\
6.1 e+01 \\
1.7 e-03\end{array}$ & $\begin{array}{r}10 \% \\
7 \%\end{array}$ \\
\hline
\end{tabular}

Customer lo Number: fea35 04 fit-20mR-10mn

Laboratory ID Number: FCB35LIQ

Hawin: Distance correction factor [100\% means no correction] 9.00\% Attenuation (Bamplo tubo)

$0.00 \%$ Attenuation (Ehisld)

Enore and MDA roported at 2 sigma

Dr. E. Fred Rladal

$3-23-95$ 


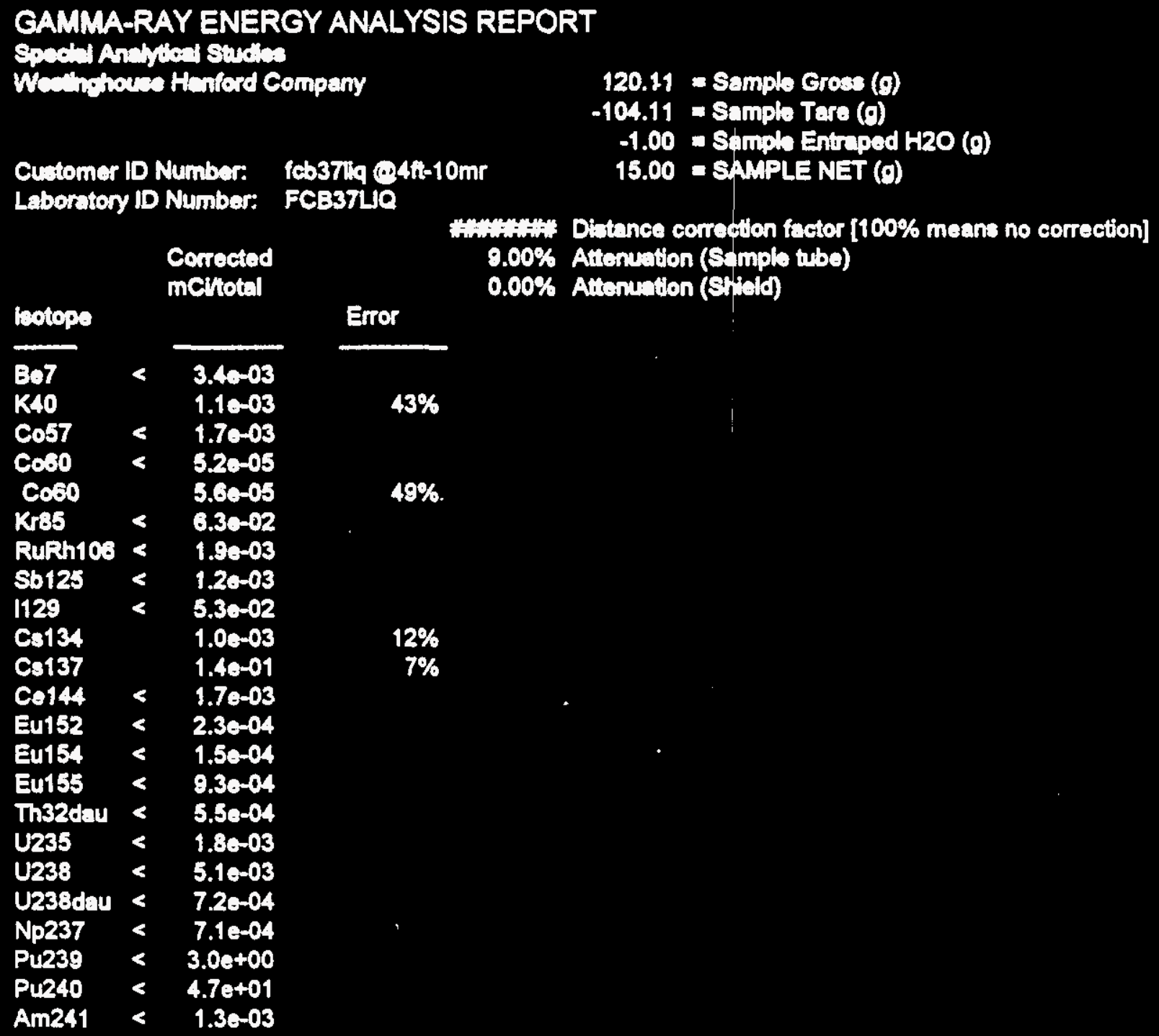

Errore and MDA reported at 2 sigma

Dr. E. Frad Risded 


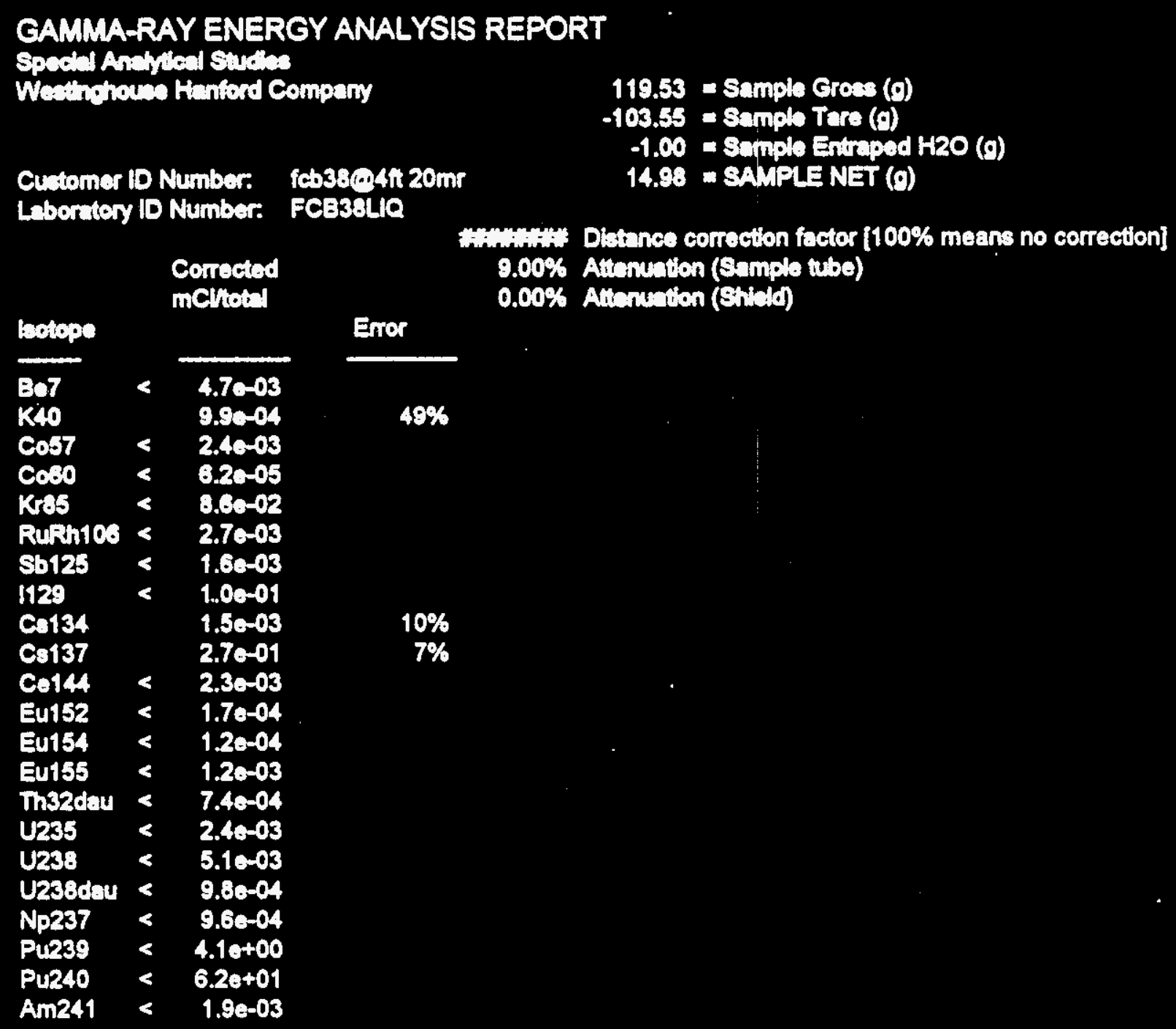

Errese and MDA reported at 2 sigma

Dr. E. Fred Nided 


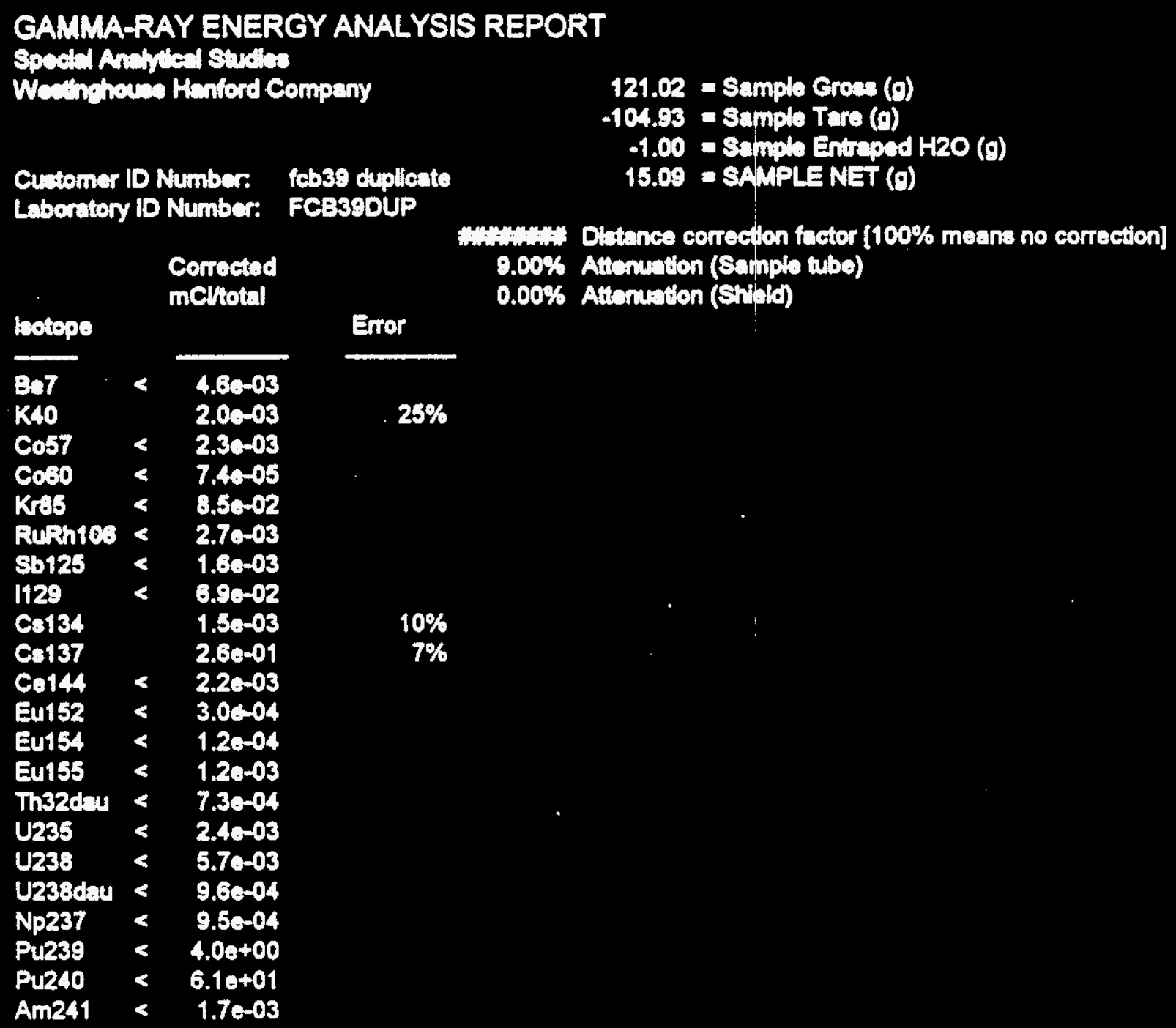

Errore and MDA reported at 2 sigma

Dr. E Fred Rtadel 
WHC-SD-SNF-DP-003, Rev. 0

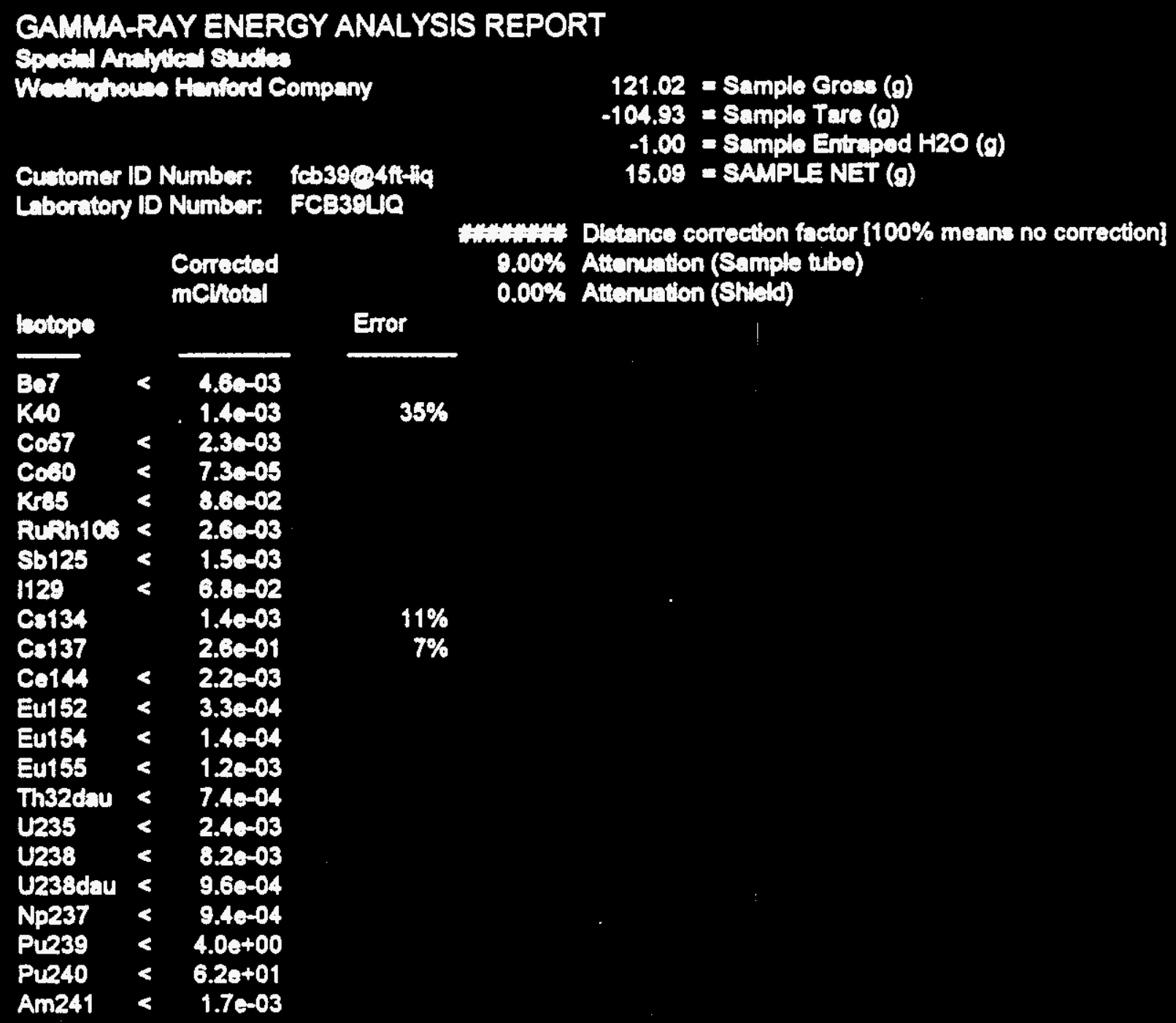

GAMMA-RAY ENERGY ANALYSIS REPORT

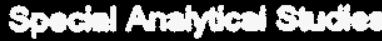

Weandiouse Honferd Company

121.02 - Sample Gross (0)

- Sample Tare (o)

15.09 - SAMPLE NET (o)

Cutomer ID Number: fob390.9ft

$0.00 \%$ Attomation (Bample tubo)

Corrected

$0.00 \%$ Attenualion (Shield)

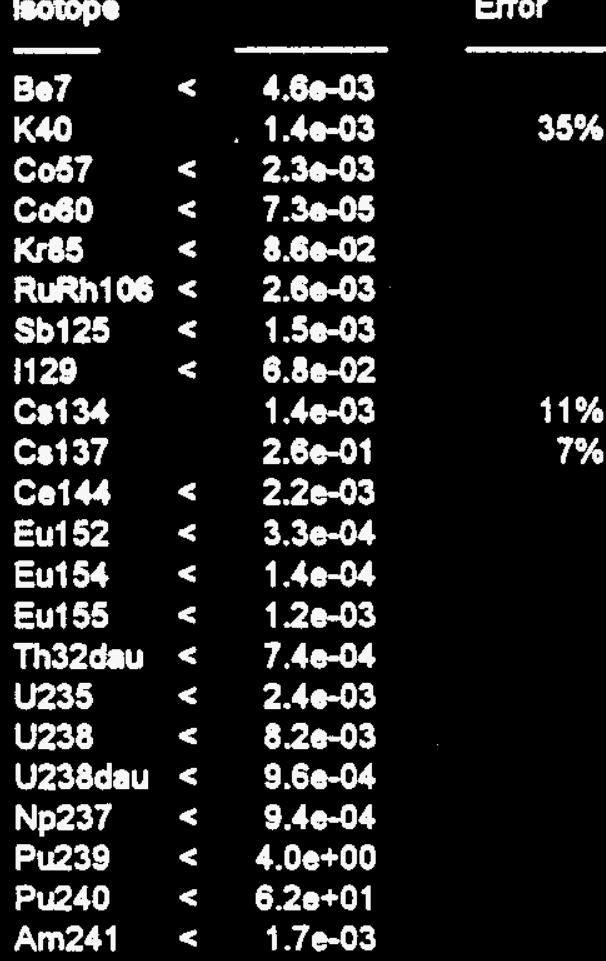

Errose and MDA reported at 2 sigma

$3-26-98$

Dr. E. Fred Raded 


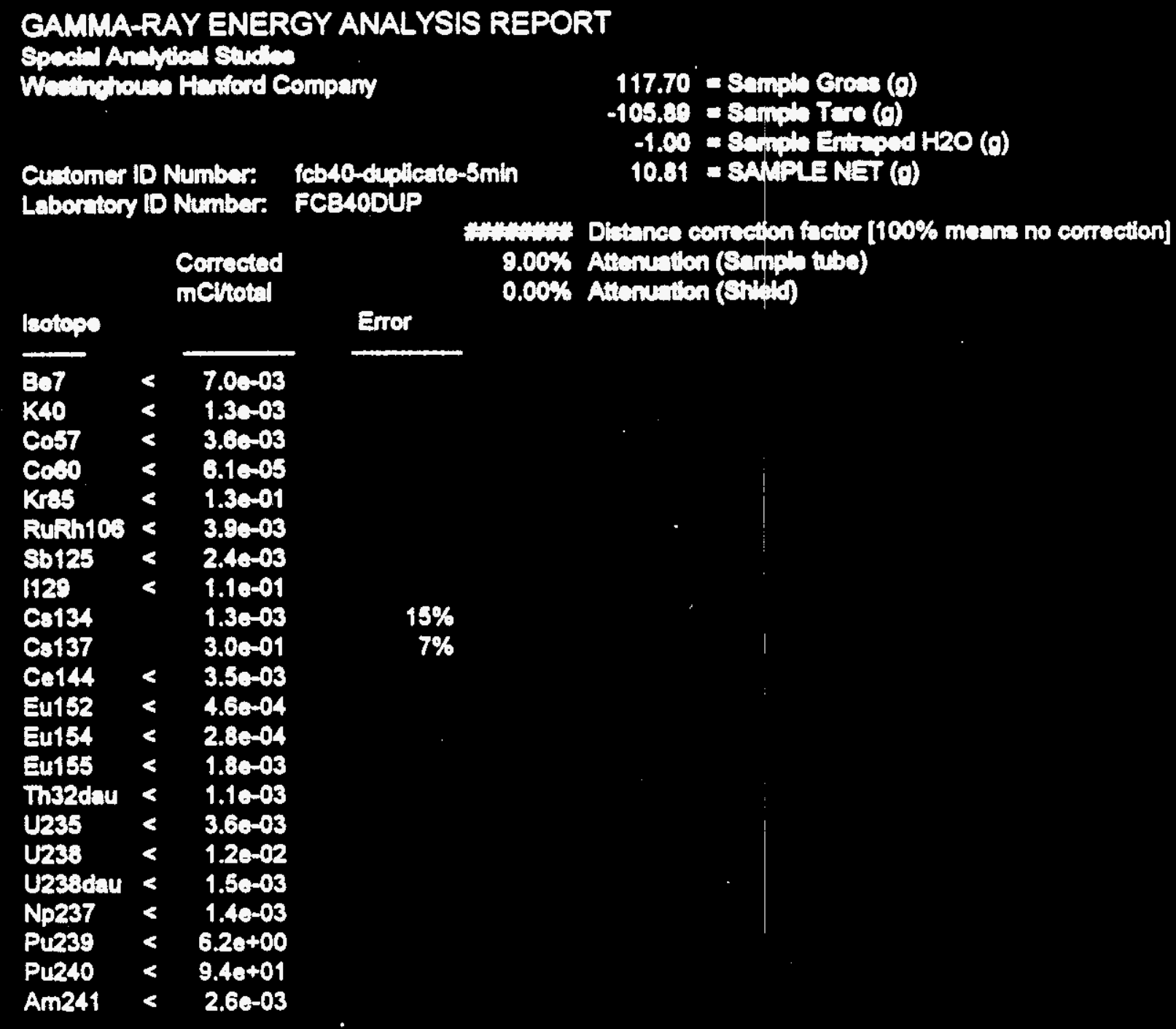

Errors and MDA reported at 2 sigme

Dr. E. Frednaded 


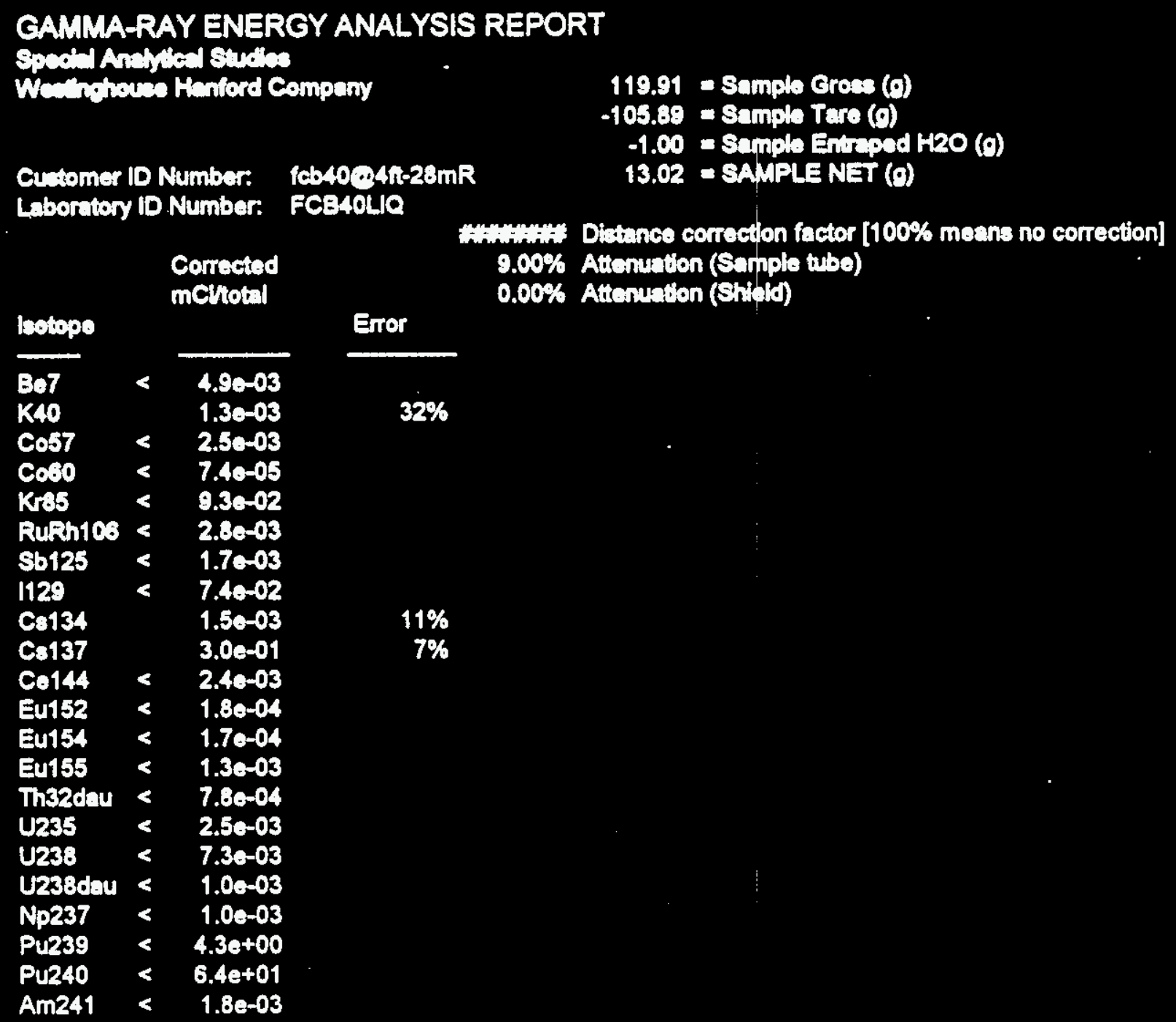

Errore and MDA reported at 2 sigme

3-26-95

Dr. E. Fred Riodd 
GAMMA-RAY ENERGY ANALYSIS REPORT

Spocid Ansyited Suctes

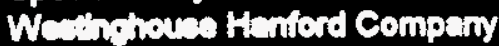

$$
\begin{aligned}
120.20 & =\text { Sample Cross }(0) \\
-105.48 & =\text { Sample Tare }(0) \\
-1.00 & =\text { Sample Entraped H2O (o) } \\
13.74 & =\text { SAMPLE NET }(0)
\end{aligned}
$$

\begin{tabular}{|c|c|c|c|}
\hline botope & & $\begin{array}{l}\text { Corrected } \\
\text { mcritotal }\end{array}$ & Error \\
\hline $\begin{array}{l}\text { Bo7 } \\
\text { K10 } \\
\text { Cost } \\
\text { Coso } \\
\text { Kres } \\
\text { RuRh10s } \\
\text { Sb125 } \\
1129 \\
\text { Cs134 } \\
\text { Cs137 } \\
\text { Ce144 } \\
\text { Eu152 } \\
\text { Eu154 } \\
\text { Eu155 } \\
\text { Th32dau } \\
\text { U235 } \\
\text { U238 } \\
\text { U238dau } \\
\text { Np237 } \\
\text { Pu239 } \\
\text { Pu240 } \\
\text { Am241 }\end{array}$ & $\begin{array}{l}< \\
< \\
< \\
< \\
< \\
< \\
< \\
< \\
< \\
<\end{array}$ & $\begin{array}{l}4.20-03 \\
1.20-03 \\
2.20-03 \\
5.90-05 \\
8.00-02 \\
2.5 e-03 \\
1.5 e-03 \\
6.40-02 \\
1.30-03 \\
2.30-01 \\
2.10-03 \\
5.40-05 \\
1.40-04 \\
1.10-03 \\
6.90-04 \\
2.30-03 \\
6.0 e-03 \\
9.10-04 \\
8.9 e-04 \\
3.8 e+00 \\
5.8 e+01 \\
1.6 e-03\end{array}$ & $\begin{array}{r}11 \% \\
7 \%\end{array}$ \\
\hline
\end{tabular}

Cuntomer ID Number: feb4104k-20mR

Laberatory ID Number: FCBA1LIe

Distance correction factor [100\% means no correction] $9.00 \%$ Attenuation (Sample tube)

$0.00 \%$ Attenuation (Shicid)

Errose and MDA roported at 2 doma

Dr. E. Fred Rodd

$3-20-95$ 
HHC-SD-SNF-DP-003, Rev. 0

CAMMA-RAY ENERGY ANALYSIS REPORT

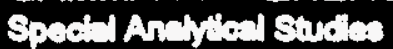

Webinchouse Hanford Company

120.19 - Sample Groas (o)

-104.12 Sample Tere (0)

-1.00 - Bampia Entraped H2O (d)

Customer ID Number: fob-12-411-17mr Laboratory ID Number: FCBAzLJa

15.07 - SAMPLE NET (o)

\begin{tabular}{|c|c|c|c|}
\hline lotbepe & & $\begin{array}{l}\text { Cerrected } \\
\text { meltotal }\end{array}$ & Error \\
\hline $\begin{array}{l}\text { But } \\
\text { K10 } \\
\text { Cos7 } \\
\text { Coso } \\
\text { Kros } \\
\text { RuRh10s } \\
\text { Sb126 } \\
1129 \\
\text { Co134 } \\
\text { Ca137 } \\
\text { Co144 } \\
\text { Eu152 } \\
\text { Eu154 } \\
\text { Eu155 } \\
\text { Th32dau } \\
\text { U235 } \\
\text { U2338 } \\
\text { U238dau } \\
\text { Np237 } \\
\text { Pu239 } \\
\text { Pu240 } \\
\text { Am241 }\end{array}$ & $\begin{array}{l}< \\
< \\
< \\
< \\
< \\
< \\
< \\
< \\
< \\
< \\
< \\
<\end{array}$ & $\begin{array}{l}3.9 e-03 \\
1.70-03 \\
2.0 e-03 \\
6.90-05 \\
7.40-02 \\
2.30-03 \\
1.36-03 \\
5.80-02 \\
1.36-03 \\
1.90-01 \\
1.90-03 \\
1.7 e-04 \\
1.70-04 \\
1.00-03 \\
6.3 e-04 \\
2.10-03 \\
8.40-03 \\
8.3 e-04 \\
8.2 e-04 \\
3.5 e+00 \\
5.4 e+01 \\
1.5 e-03\end{array}$ & $\begin{array}{r}11 \% \\
7 \%\end{array}$ \\
\hline
\end{tabular}

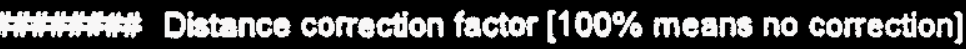
$9.00 \%$ Attenuation (8ample tube)

$0.00 \%$ Attenuation (Shield)

$1 \%$

$\%$

Errose and MDA reperted at 2 eismo

$3-20-98$

Dr. E. Fred Rised 
CAMMA-RAY ENERGY ANALYSIS REPORT

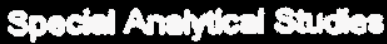

Wertuthawe Honferd Company

121.64 - Sampla Groas (o)

-105.72 - Sample Tare (o)

-1.00 - Sample Entraped H2O (O)

Cutomer ID Number: fob 3304 4h-20mR 14.92 - SAMPLENET (o)

Laboratory ID Number: FCBA3LIQ

\begin{tabular}{|c|c|c|c|}
\hline betope & & $\begin{array}{l}\text { Correeted } \\
\text { neltotal }\end{array}$ & Error \\
\hline $\begin{array}{l}\text { Bo7 } \\
\text { K40 } \\
\text { Cos7 } \\
\text { Co60 } \\
\text { Kros } \\
\text { RuRh10s } \\
\text { Sb125 } \\
\text { I129 } \\
\text { Co134 } \\
\text { Cs137 } \\
\text { Ce144 } \\
\text { Eu152 } \\
\text { Eu154 } \\
\text { Eu155 } \\
\text { Th32dau } \\
\text { U235 } \\
\text { U238 } \\
\text { U238dau } \\
\text { Np237 } \\
\text { Pu239 } \\
\text { Pu240 } \\
\text { Am241 }\end{array}$ & $\begin{array}{l}< \\
< \\
< \\
< \\
< \\
< \\
< \\
< \\
< \\
<\end{array}$ & $\begin{array}{r}4.4 e-03 \\
1.3 e-03 \\
2.3 e-03 \\
9.40-05 \\
8.30-02 \\
2.6 e-03 \\
1.5 e-03 \\
9.80-02 \\
1.4 e-03 \\
2.5 e-01 \\
2.2 e-03 \\
2.3 e-04 \\
9.1 e-05 \\
1.20-03 \\
7.0 e-04 \\
2.3 e-03 \\
6.5 e-03 \\
9.6 e-04 \\
9.3 e-04 \\
3.8 e+00 \\
6.0 e+01 \\
1.8 e-03\end{array}$ & $\begin{array}{r}11 \% \\
7 \%\end{array}$ \\
\hline
\end{tabular}

Hanuivi: Dlatance correction factor [100\% means no correction] $9.00 \%$ Attonuation (Sample tubo) $0.00 \%$ Attemuation (Ehisid)

Errose and MDA reported at 2 aloma

Dr. E. Fred Rided 
GAMMA-RAY ENERGY ANALYSIS REPORT

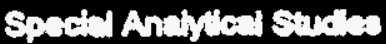

Wemathora Heriford Company

$$
\begin{aligned}
& 103.05 \text { - Sample Gross (o) } \\
& -104.04=\text { Sample Tare (o) } \\
& -1.00 \text { a sampio Entraped H2O (o) } \\
& 1.01 \text { - SANPLE NST (o) }
\end{aligned}
$$

Customer ID Number: fob36@ $2 i n,<.5 \mathrm{mR}$ Laboratory ID Number: FCB36CAS

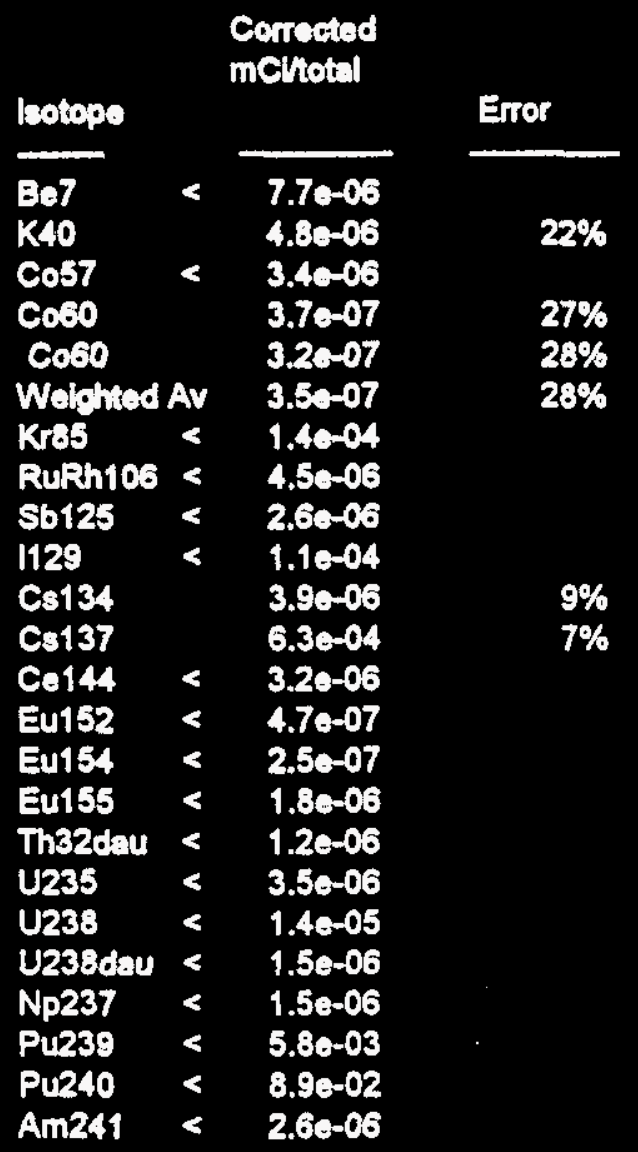

12.50\% Dibtance correction factor [100\% means no correction] $9.00 \%$ Attenuation (Bample tubo) $0.00 \%$ Attenuation (Ehicid)

Dr. E. Fred Rivil 
GAMMA-RAY ENERGY ANALYSIS REPORT

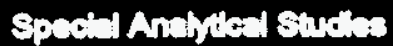

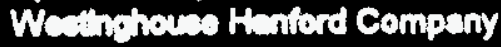

$$
\begin{aligned}
& 118.08 \text { - Sample Grose (a) } \\
& -104.81 \text { - Sumple Tare (o) } \\
& -1.00 \text { a sumph Entroped H2O (o) } \\
& 1227 \text { - SAMPLE NET (d) }
\end{aligned}
$$

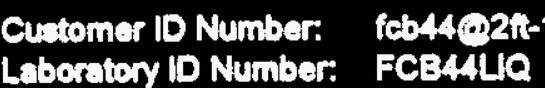

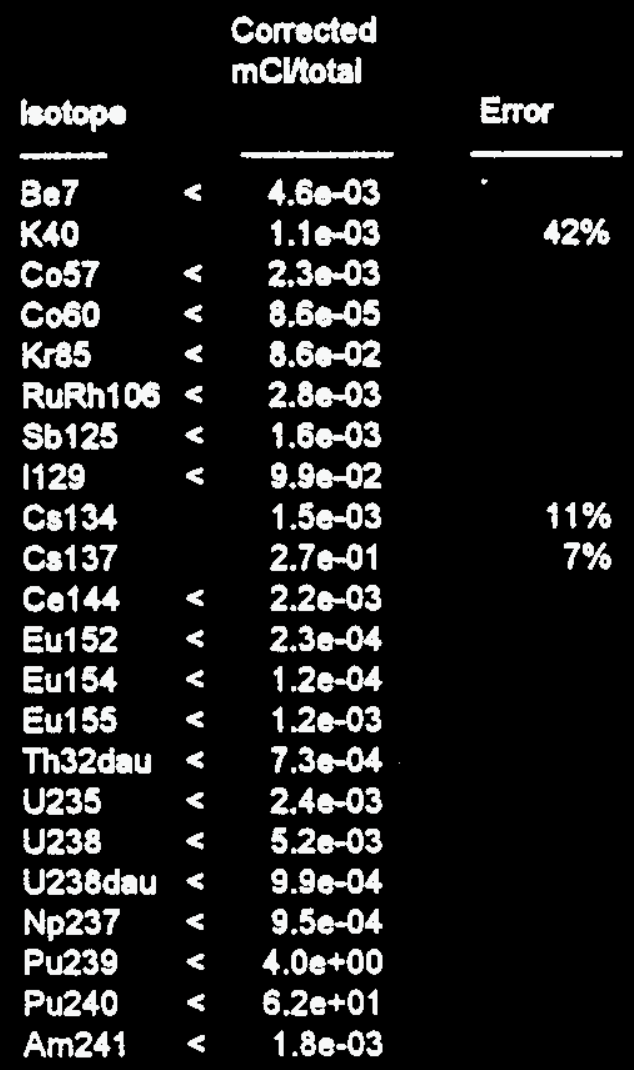

\subsection{0\% Attenuation (Eamplo trbe)}

$0.00 \%$ Attemution (Shils)

Errors and MDA reperted at 2 siama

Dr. E. Fred Rided 
GAMMA-RAY ENERGY ANALYSIS REPORT

Spocial Antrind Sackeo

Weringhose Hentord Company

$$
\begin{aligned}
117.87 & =\text { Sample Croas }(0) \\
-102.50 & =\text { Sample Tare }(0) \\
-1.00 & =\text { Sample Entrad H2O (o) } \\
14.37 & =\text { SAMPLE NET }(0)
\end{aligned}
$$

\begin{tabular}{|c|c|c|c|}
\hline botepe & & $\begin{array}{l}\text { Corrected } \\
\text { nchotal }\end{array}$ & Error \\
\hline $\begin{array}{l}\text { Bo7 } \\
\text { K40 } \\
\text { Cos7 } \\
\text { Coco } \\
\text { Kras } \\
\text { Ruph106 } \\
\text { Sb125 } \\
1129 \\
\text { Co134 } \\
\text { Co137 } \\
\text { Ce144 } \\
\text { Eu152 } \\
\text { Eu154 } \\
\text { Eu155 } \\
\text { Th32dou } \\
\text { U235 } \\
\text { U238 } \\
\text { U238dau } \\
\text { Np237 } \\
\text { Pu239 } \\
\text { Pu240 } \\
\text { Am241 }\end{array}$ & $\begin{array}{l}< \\
< \\
< \\
< \\
< \\
< \\
< \\
< \\
< \\
< \\
<\end{array}$ & $\begin{array}{l}5.0 e-03 \\
9.40-04 \\
2.5 e-03 \\
6.6 e-05 \\
9.6 e-02 \\
3.10-03 \\
1.8 e-03 \\
1.10-01 \\
1.9 e-03 \\
3.3 e-01 \\
2.40-03 \\
2.7 e-04 \\
1.70-04 \\
1.3 e-03 \\
8.16-04 \\
2.6 e-03 \\
6.6 e-03 \\
1.1 e-03 \\
1.0 e-03 \\
4.3 e+00 \\
6.6 e+01 \\
2.0 e-03\end{array}$ & $\begin{array}{r}10 \% \\
7 \%\end{array}$ \\
\hline
\end{tabular}

Customer ID Number: fob45@4ti 13mR

Laberatery ID Number: FCE4SLJa

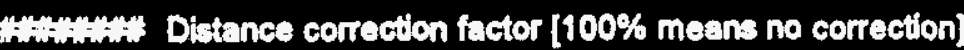
9.00\% Attenuation (Sample tube)

$0.00 \%$ Amenuation (Shiold)

Enore and MaA reported at 2 sima

$3-27.95$

Dr. ERrodradd 
WHC-SD-SNF-DP-003, Rev. 0

\section{A P P E N D IX B}

\section{PMess I THBover IV MO 1 THROVAy 53 OF}

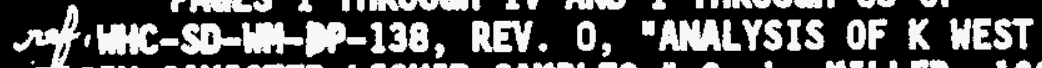
BSTI CMISTER LIOUID SHPLES," G. L. MILLER, 1995,

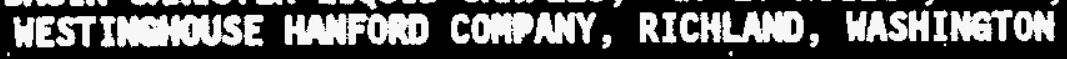


WHC-SD-SNF-DP-003, Rev. 0

\section{SUPPORTING DOCUMENT}

1. Total Pages

2. Titio

Analysis of $K$ West Basin Canister Liquid Samples

3. Number sef,

4. Rov Mo.

5. Koy Worde

WHC-SD-WM-OP- 138

Analysis, $K$ West, $K$ West Basin, Canister, Liquid

6. Author

Samples

Nane: George L. Miller

Hudruée

signifure

Orgenization/charge code 75970/MDR21

7. Abatract

$N / A$

8. release stanp

(2)

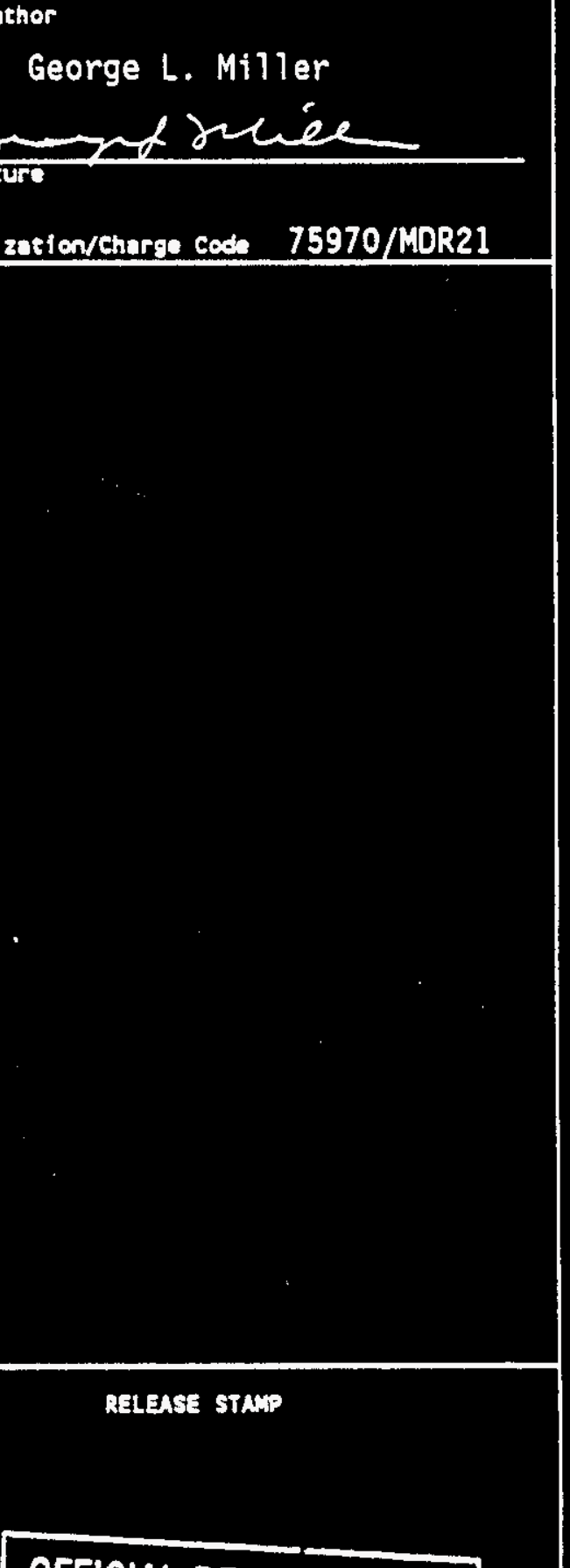

OFFICIAL RELEASE BY WHC 


\section{AMALYSIS OF $K$ WEST BASIN CANISTER LIQUID SAMPLES}




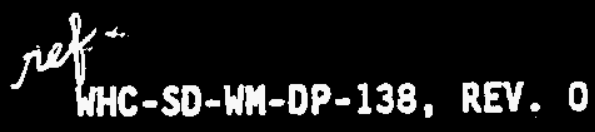

TABLE OF CONTENTS

Marrative ............................... 1

Sample Data Sumary . . . . . . . . . . . . . . . . . . . 13

Labcore Formatted Sumary Tables . . . . . . . . . . . . . 14

Excel Formatted Sunary Tabios . . . . . . . . . . . . . . 40

Chath of Custody Forms . . . . . . . . . . . . . . . . . . 54

Correspondence . . . . . . . . . . . . . . . . . . . . . . 60

Sample Preparations . . . . . . . . . . . . . . . . . . . 63

Acid Digestions Worklist 1262 . . . . . . . . . . . . . . 64

Acid Digestions Worklist 1267 ..................... 65

Acid Digestions Workl ist 1298 . . . . . . . . . . . . . . . 66

Acid Digestions Worklist 1369 . . . . . . . . . . . . . . . 67

Appearance Korklist 1209 . . . . . . . . . . . . . . . 68

Appearance Horklist 1210 . . . . . . . . . . . . . . . . 69

Appoarance Worklist 1211 . . . . . . . . . . . . . . . . 70

Appearance Worklist 1212 . . . . . . . . . . . . . . . 71

Appearance Workl ist 1347 . . . . . . . . . . . . . . . . 72

Inorganic Analyses . . . . . . . . . . . . . . . . . . . 74

pH Analysis (by Ion Selective Electrode) Worklist *1352 . . . . . 75

pH Analysis (by Ion Selective Electrode) Workl ist 1353 . . . . . . 77

pH Analysis (by Ion Solective Electrode) Workl ist $\$ 1430$. . . . . . 79

pli Analysis (by Ion Selective Electrode) Workl ist 1515 . . . . . . 80

Amenia (by Distillation/Ion Selective Electrode) Horkl ist \$ 565 . . 81 Aunonia (by Distlliation/Ion Selective Electrode) Workl ist 760 . . 96 Amilonia (by Distiliation/Ion Selective Electrode) Norklist 1429 . 110

Ion Chromatography (Witrate/Nitrite) Worklist 1328 . . . . . 126

Ion Chromatography (Witrate/Nitrite) Worklist 1329 . . . . . . . 147

Ion Chrouatography (Witrate/Nitrito) Worklist \#1355 . . . . . . 163

Ion Chromatography (Witrate/Nitrito) Worklist 1435 . . . . . . . 178

Ion Chromatography (Nitrate/Nitrite) Worklist \$ 1524 . . . . . . . 193

Potassium (by Inductively Coupled Plasma/Optical Emision

Spectroscopy) Workl ist 1315 . . . . . . . . . . . 204

Potassiun (by Inductively Coupled Plasma/Optical Enimision

Spectroscopy) Workl ist 1317 .............. 229 
SHC-SD-NH-DP-138, REY. 0

TABLE OF CONTENTS(Continued)

Potassiun (by Inductively Coupled Plasma/Optical Emision Spectroscopy) Workl ist " 1398 . . . . . . . . . . . . . 255

Potassium (by Inductively Coupled Plasma/Optical Emmision Spectroscopy) Worklist 1408............... 283

Radtochentcal Analyses .................. 310

Uranium (by Laser Induced Kinetic Phophorescence) Norkl ist \#1341 • 311

Uranium (by Laser Induced Kinotic Phophorescence) Horki ist *1342 • 315

Uranium (by Laser Induced Kinotic Phophorescence) Workl ist 1557 . 323

Strontium 89/90 (by Separation/Proportional Counting)

Norkl ist $\$ 1324$. . . . . . . . . . . . . . . 325

Strontium $89 / 90$ (by Separation/Proportianal Counting)

Norkl tst 1326 ... . . . . . . . . . . 339

Strontium $89 / 90$ (by Separation/Proportional Counting)

Norkl ist \# $1327 . \ldots . . . . . . . . . .353$

Strontium $89 / 90$ (by Separation/Proportional Counting)

Norklist \# 1350 (by Separation/Proportionai Counting)

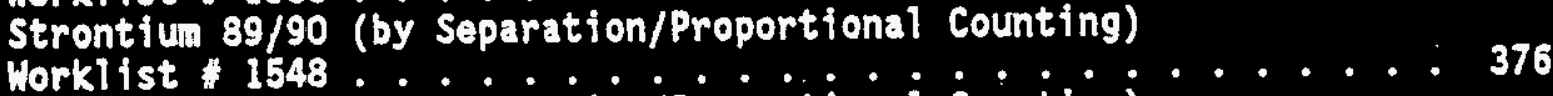

Stront um $89 / 90$ (by Separation/Proportional Counting)

Norklist * 1549

Tritium (by Lachat Micro-0istillation/Liquid

Scintillation Counting) Norkl ist $\$ 1333 \ldots . . . . . .399$

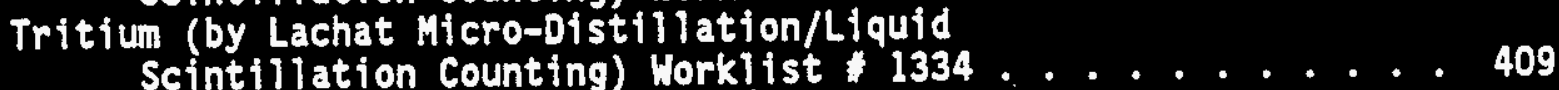

Tritium (by Lachat Micro-Distillation/Liquid
Scintillation Counting) Workl ist $1556 \ldots 424$

Garma Energy Analys is (GEA)

GEA (Cesiun-137 and Amertciun-241) Worklist *1312 .......431

GEA (Cosiun-137 and Amoriciun-241) Work1 ist $1313 \ldots 473$

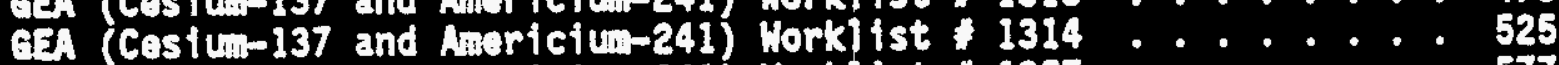

eAA (Cestum-137 and Americtum-241) Workl ist $1357 \ldots \ldots$. . . . 577

eEA (Cesium-137 and Americium-241) Workl ist 11820 . . . . . 609

Anericium-241 (by Extraction/Alpha Energy Analysis -AEA)

Morkl ist *1387. . . . . . . . . . . . 637

Amertcium-241 (by Extraction/Aipha Energy Analysis -AEA)

Morkl ist 1388 . . . . . . . . . . . . . . 654

Amariciun-241 (by Extraction/Aipha Energy Analys is -AEA)

Workl ist \# 1389 
$M$

WHC-SD-Wh-DP-138, REV. O

TABLE OF CONTENTS(Continued)

Amariciun-241 (by Extraction/Alpha Energy Analysis -AEA)

Workl ist 1390 . . . . . . . . . . . . . . . . . . 707

Americiun-241 (by Extraction/A1pha Energy Analysis -AEA) Workl ist :

1465

Plutoniui-239/240 (by Ion Exchange/A1pha Energy Anaiysis-AEA)

Morkl tst 1383 . . . . . . . . . . . . . . . . 755

Plutonitu-238 (By Ion Exchange/Alpha Energy Analysts-AEA)

Nork1 ist 1384 . . . . . . . . . . . . . . . . . . 774

Plutontua-238 (By Ion Exchange/A1 pha Energy Analysis-AEA)

Workl tst 1385 ..................... . 800

Plutoniu-238 (By Ion Exchange/A1pha Energy Analysis-AEA)

Workl tst 1386 . . . . . . . . . . . . . . . . 826

Plutontum-238 (By Ion Exchange/Alpha Energy Analysis-AEA)

Worklist 1424 . . . . . . . . . . . . . . . . . . 852

Plutonium-239/240 (by Ion Exchange/A1pha Energy Analys is-AEA)

Workl ist $1464 . . . . . . . . . . . . . . . . . . .865$

This documant consists of pages 1 through 886 . 
sof-

WHC-SD-WH-DP-138, REY. 0

NARRATIVE 


\title{
ANALYSIS OF $\mathrm{K}$ WEST BASIN CANISTER LIQUID SAMPLES
}

\author{
CASE NARRATIVE
}

\section{INTROOUCTION}

\section{Project Documentation and Direction}

Analytical direction for this project was provided in the following references.

1. Harris, R. A., et a1., WHC-SD-SNF-PLN-004, Rev. 0, released February 28, 1995, Westinghouse Hanford Company, Richland, Washington, "Sampling and Analys is Plan for Canister Liquid and Gas Sampling at 105-K West Fuel Storage Basin".

2. Trimble, D. J., WHC-SD-SNF-PLN-004, Rev. O-A, ECN 168171, released April 14, 1995, Westinghouse Hanford Company, Richland, Washington, "Sampling and Analys is Plan for Canister Liquid and Gas Sampling at 105-K West Fuel Storage Basin".

3. Trimble, D. J., Internal Meno to G. L. Miller, April 11, 1995, Westinghouse Hanford Company, "Instructions for Analys is of $K$ West Basin Canister Liquid Samples".

4. Trimble, D. J., Internal Memo to G. L. Miller, April 18, 1995, Westinghouse Hanford Company, "Revised Instructions for Analysis of K West Basin Canister Liquid Samples".

5. Trimble, D. J., cc:Mail message to G. L. Miller, May 5, 1995, Westinghouse Hanford Company, "K Basin Sample Priorities".

\section{Sample Collection}

Twenty-six water samples were collected from 105-K West Basin fuel canisters between March 15 and March 27, 1995. A document furnished by the program indicated that the samples ranged in weight (approximate volume in milliliters) from 4.88 to 17.13 grams.

\section{Sample Receipt}

The samples were received in two shipments by the 222-S Laboratory on April 20 and May 4, 1995, for inorganic and radiochemical analyses. All samples were logged into the laboratory using the LABCORE laboratory information management system (LIMS). 
<smiles>C[Si]1(C)CC1</smiles>

WHC-SD-WM-DP-138, REV. 0

\section{Quality Control}

The program specified that no less than 10 percent of the samples should be evaluated for precision and accuracy, wherever possible. Control 1 imits for precision or accuracy were specified by the program in Reference 2 . To determine analytical precision, analyses were performed in triplicate for all analytes on three samples except for visual appearance. To determine analytical accuracy, three samples were spiked for the following analytes:

$\begin{array}{ll}- & 239 / 240 \mathrm{Pu} \\ & { }^{241} \mathrm{Am} \\ & { }^{90} \mathrm{Sr} \\ \text { - tritium } \\ \quad \text { total uranium } \\ \text { - } \quad \text { ammonia } \\ \text { - nitrate } \\ \quad \text { nitrite. }\end{array}$

Samples which were spiked were the same as those for which replicate precision determinations were performed.

No criteria were specified by the program for maximum sample holding time.

Acid digestions were performed on the samples prior to analys is of ${ }^{239 / 240} \mathrm{Pu}$, 24 Am and potassium.

Because of the small original volume of each of the samples, it was necessary to use less than the optimum analytical aliquot for many analyses, conserving sufficient sample to analyze for all of the specified analytes. Despite this effort to conserve sample, two samples were unable to be analyzed for ${ }^{90} \mathrm{Sr}$ due to insufficient sample. A second issue related to using less than the optimum analytical aliquot was that reported values for potassium, ammonia, nitrate, nitrite and 261 Am by gama energy analys is were raised significantly and, in many cases, were greater than the program's required practical quantitation 1 imit (PQL).

\section{SANPLE IDENTIFICATION}

Upon receipt of samples at 222-5 Laboratory, new sample numbers were assigned to the samples. Using LABCORE to $\mathrm{log}$ in the samples, original program sample numbers were converted to laboratory sample numbers. Additional laboratory sample numbers were assigned to the acid digestion subsets of samples. Table 1 relates the sample identifications assigned by the laboratory to those of the program. 
WHC-SD-WM-DP-138, REV. 0

Table 1. Sample Identification

\begin{tabular}{|c|c|c|c|c|}
\hline $\begin{array}{l}\text { Program Sample } \\
\text { Identification }\end{array}$ & $\begin{array}{l}\text { Lab Sample I0 } \\
\text { (direct analysis) }\end{array}$ & $\begin{array}{l}\text { Lab Sample ID } \\
\text { (acid digestion) }\end{array}$ & $\begin{array}{c}\text { Sample } \\
\text { Collection } \\
\text { Date/Time } \\
\end{array}$ & $\begin{array}{c}\text { Lab Receipt of } \\
\text { Sample } \\
\text { Date/Time }\end{array}$ \\
\hline $\mathrm{FC}-\mathrm{BO2}$ & S95K0000033 & $595 K 000040$ & $3 / 15 / 95,1945$ & $5 / 4 / 95,1025$ \\
\hline$F C-807$ & S95K000034 & $595 K 000041$ & $3 / 17 / 95,1855$ & $5 / 4 / 95,1025$ \\
\hline$F C-B 10$ & S95K000035 & $595 K 000042$ & $3 / 18 / 95, \quad 1058$ & $5 / 4 / 95, \quad 1025$ \\
\hline $\mathrm{FC}-\mathrm{B} 14$ & $595 K 000047$ & $595 K 000049$ & $3 / 20 / 95,2110$ & $5 / 4 / 95,1025$ \\
\hline$F C-B 15$ & $595 \times 000036$ & $595 K 000043$ & $3 / 20 / 95,2145$ & $5 / 4 / 95, \quad 1025$ \\
\hline $\mathrm{FC}-\mathrm{B} 16$ & $595 K 000037$ & S95K000044 & $3 / 21 / 95,1020$ & $5 / 4 / 95, \quad 1025$ \\
\hline $\mathrm{FC}-\mathrm{B} 17$ & $595 \times 000038$ & S95K000045 & $3 / 21 / 95,1050$ & $5 / 4 / 95, \quad 1025$ \\
\hline$F C-B 18$ & $595 K 000039$ & S95K000046 & $3 / 21 / 95,1315$ & $5 / 4 / 95, \quad 1025$ \\
\hline$F C-B 19$ & $595 \mathrm{~K} 000001$ & S95K000002 & $3 / 21 / 95, \quad 1335$ & $4 / 20 / 95,1400$ \\
\hline $\mathrm{FC}-\mathrm{B} 21$ & $595 K 000051$ & $\mathrm{n} / \mathrm{a}$ & $3 / 24 / 95, \quad 1048$ & $5 / 4 / 95,1025$ \\
\hline $\mathrm{FC}-\mathrm{B} 23$ & $595 K 000048$ & $595 K 000050$ & $3 / 24 / 95,1918$ & $5 / 4 / 95,1025$ \\
\hline $\mathrm{FC}-\mathrm{B24}$ & S95K000025 & $595 K 000030$ & $3 / 24 / 95,2108$ & $4 / 20 / 95,1400$ \\
\hline$F C-B 25$ & $595 K 000005$ & S95K000007 & $3 / 24 / 95,2200$ & $4 / 20 / 95,1400$ \\
\hline $\mathrm{FC}-\mathrm{B} 26$ & $595 K 000009$ & S95K000016 & $3 / 24 / 95,2230$ & $4 / 20 / 95,1400$ \\
\hline$F C-B 29$ & $595 K 000010$ & S95K000017 & $3 / 25 / 95,1315$ & $4 / 20 / 95,1400$ \\
\hline$F C-B 30$ & S95K000026 & $595 \mathrm{~K} 000031$ & $3 / 25 / 95,1331$ & $4 / 20 / 95,1400$ \\
\hline $\mathrm{FC}-\mathrm{B3} 5$ & S95K0000II & S95K000018 & $3 / 25 / 95,2150$ & $4 / 20 / 95,1400$ \\
\hline $\mathrm{FC}-\mathrm{B37}$ & S95K000012. & S95K000019 & $3 / 26 / 95,1128$ & $4 / 20 / 95,1400$ \\
\hline $\mathrm{FC}-838$ & $595 K 000027$ & 595K000032 & $3 / 26 / 95,1145$ & $4 / 20 / 95,1400$ \\
\hline$F C-B 39$ & S95K000013 & $595 K 000020$ & $3 / 26 / 95, \quad 1220$ & $4 / 20 / 95,1400$ \\
\hline$F C-840$ & $595 K 000023$ & S95K000028 & $3 / 26 / 95, \quad 1215$ & $4 / 20 / 95,1400$ \\
\hline$F C-B 41$ & $595 K 000014$ & 5956000021 & $3 / 26 / 95,1416$ & $4 / 20 / 95,1400$ \\
\hline $\mathrm{FC}-842$ & S95K000015 & S95K000022 & $3 / 26 / 95,1440$ & $4 / 20 / 95,1400$ \\
\hline $\mathrm{FC}-\mathrm{B} 43$ & $595 K 000003$ & $595 K 000004$ & $3 / 26 / 95,1450$ & $4 / 20 / 95,1400$ \\
\hline $\mathrm{FC}-\mathrm{B} 44$ & S95K000006 & 595K0000008 & $3 / 27 / 95, \quad 1322$ & $4 / 20 / 95,1400$ \\
\hline $\mathrm{FC}-\mathrm{B} 45$ & S95K000024 & S95K000029 & $3 / 27 / 95,1349$ & $4 / 20 / 95,1400$ \\
\hline
\end{tabular}




\section{RESULTS OF ANALYSIS}

\section{Report Format}

Interim data were provided to the program each week during sample analyses. These data were tentative and subject to change pending review of data correctness, completeness and pending possible reruns to satisfy program quality control requirements. These reports were generated using a standard report format from the LABCORE system. LABCORE, a new computerized laboratory information management systen, is in the process of being implemented in the laboratory, and still has many bugs to be fixed. Consequently, the reports generated by LABCORE omitted some data and reported some erroneous values. LABCORE's reported values are slightly different than the originally derived data for several parameters because it rounds the values, makes additional computations, and then rounds the data again. Although these systematic errors are known and understood, they are not currently able to be fixed because they are part of the software developer's proprietary source code.

For this final report, two report formats were used to provide summary data tables: standard LABCORE printouts and Exce $]^{\mathrm{m}}$. spreadsheets.

Finai LABCORE reports were generated, pages 14 through 39 , where all of the analytes were displayed by sample location. For the final report, data in the LABCORE database were corrected or data were added to generate a report as complete as possible. The standard LABCORE format does not include results of triplicate analyses and does not include triplicate results in the calculated "average" value. The standard LABCORE format reports sample precision as relative percent difference (RPD) between the sample and its duplicate rather than as two times the relative standard deviation (2 RSD) of the sample, duplicate and triplicate, as specified for this project. The LABCORE report format does not display information on sample appearance, but only states that the analysis was "complete."

A second set of report tables was generated as Excelm spreadsheets, where the data for all samples were displayed by each analyte. Because there was control over the format and contents of these tables, they accurately represent the true and complete analytical data. These tables are shown on pages 40 through 53 .

$m=$ Excel is a registered trademark of Microsoft Corporation 


$$
\text { nof. }
$$

Discussion of Results

\section{Sample Appearance and Dose Rate}

All samples were observed visually at the time of sample breakdown on April 26 and May 10, 1995. Each was determined to be colorless, clear and to not have solids present. The chemist's comment for several samples included, "no organic layer," but this observation was not recorded for several other samples. The geometry-corrected contact dose rates for the samples, measured at the time of visual inspection, ranged from 15 to $150 \mathrm{mRad} /$ hour. No quality control analyses are required for visual inspections.

\section{pH Analysis by Ion Selective Electrode}

Samples were analyzed for $\mathrm{pH}$ using procedure $L A-212-102$, revision $\mathrm{C}-5$, on May 12, 23 and June 7, 1995. No procedural anomalies occurred during the analyses of $\mathrm{pH}$, and there were no technical difficulties. All sample pH values were greater than 7, except for sample FC-BO2, which had a result of 3.81 .

Analytical accuracy was determined on the basis of a control standard. The percent recoveries of the control standard ranged from 99.7 to 100.5 , and were within the program's specified control limits of $100 \pm 10$ percent recovery. The spiking of samples for $\mathrm{pH}$ is technically not possible, consequently no spiked analyses were performed.

Analytical precision was evaluated on three samples, where the analyses were performed in triplicate. Precision was calculated as 2 times the relative standard deviation of the three replicate values for a sample. The precision results ranged from 0.00 to 0.39 RSD, which were within the program's specified control limits of \pm 10 RSD.

\section{Almonia by Distillation/Ion Selective Electrode}

Samples were analyzed for ammonia using procedure $L A-631-001$, revision $A-0$, on May 9, 10, 31 and June 1, 1995. No procedural anomalies occurred during the analyses, and there were no technical difficulties. All samples were less than the detection 1 imit except for sample $F C-B 39$, which had a result only slightly greater than the detection limit.

Analytical accuracy was determined on the basis of a spiked sample. The spike percent recoveries ranged from 95.6 to 100.0 , and were within the program's specified control limits of $100 \pm 20$ percent recovery. Ammonia control standards performed well, with results ranging from 92.0 to 105.0 percent recovery.

Analytical precision was evaluated on three samples, where the analyses were performed in triplicate. Precision was calculated as 2 RSD of the three 


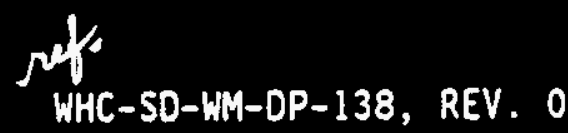

replicate values for a sample. Precision results were unable to be calculated because all analytical results were less than the detection 1 imit.

Reagent blanks were analyzed in each batch of samples. No contamination was detected in any of the blanks. Many of the blank results were reported as values less than the detection limit for the sample. This is possible because blanks are run undiluted and the samples were diluted. The dilution factor is multiplied times the instrument detection 1 imit when calculating the final sample detection limit.

\section{Nitrate by Ion Chromatography}

Samples were analyzed for nitrate using procedure LA-533-105, revision C-2, on May 9,10 and June 6 , and 7, 1995. No procedural anomalies occurred during the analyses, and there were no technical difficulties. Samples results ranged from less than the detection 1 imit to $69.3 \mu \mathrm{g} / \mathrm{ml}$.

Analytical accuracy was determined on the basis of spiked samples. The spike percent recoveries ranged from 94.0 to 113.2, and were within the program's specified control 1 imits of $100 \pm 20$ percent recovery. Nitrate control standards performed wel1, with results ranging from 95.7 to 101.2 percent recovery.

Analytical precision was evaluated on three samples, where the analyses were performed in triplicate. Precision was calculated as 2 RSD of the three replicate values for a sample. The calculable precision results ranged from 1.5 to 8.4 RSO, which were within the program's specified control limits of t20 RSD. Precision RSD was not able to be calculated for sample FC-B35 because all results were less than the detection limit.

No contamination was observed in any of the reagent blanks. One reagent blank was analyzed in each batch of samples.

\section{Nitrite by Ion Chromatography}

Samples were analyzed for nitrite using procedure LA-533-105, revision $6-2$, on May 9,10 and June 6 , and 7,1995 . No procedural anomal ies occurred during the analyses, and there were no technical difficulties. Sample results ranged from less than the detection 1 imit to $4.28 \mathrm{\mu g} / \mathrm{ml}$.

Analytical accuracy was determined on the basis of spiked samples. The spike percent recoveries ranged from 89.7 to 100.5 , and were with in the program's specified control 1 imits of $100 \pm 20$ percent recovery. Nitrite control standards performed well, with results ranging from 95.6 to 100.0 percent recovery.

Analytical precision was evaluated on three samples, where the analyses were performed in triplicate. Precision was calculated as 2 RSO of the three 
NHC-SD-WM-DP-138, REV. 0

replicate values for a sample. The calculable precision result for $F C-B 23$ was 3.7 RSD, and was with in the program's specified control limits of \pm 20 RSD. Precision RSD was not able to be calculated for samples FC-829 and FC-B35 because all results were less than the detection limit.

No contamination was observed in any of the reagent blanks. One reagent blank was analyzed in each batch of samples.

\section{Potassium by Inductively Coupled Plasma/Optical Emission Spectroscopy}

Samples were acid digested on May 2, 5, 10 and 22, 1995, using procedure LA505-158, revision A-4, and then anaiyzed for potassium using procedure LA-505151, revision D-2, on May 19 and 24, 1995. No procedural anomalies occurred during the preparations or analyses, and there were no technical difficulties. Sample results ranged from less than the detection 1 imit to $60.9 \mu \mathrm{g} / \mathrm{ml}$. As discussed above in Quality Control, the program's required $\mathrm{PQL}$ of $1 \mathrm{\mu g} / \mathrm{mi}$ was not able to be met. The reported detection limit was 30 times greater than the required $\mathrm{PQL}$ with a value of $30 \mu \mathrm{g} / \mathrm{ml}$.

Analyticat accuracy was determined on the basis of five spiked samples. The spike percent recoveries ranged from 98.2 to 124.4. Four of the five spiked results were within the program's specified control 1 imits of $100 \pm 20$ percent recovery. Nitrate control standards performed well, with results ranging from 94.8 to 103.3 percent recovery.

Analytical precision was evaluated on three samples, where the analyses were performed in triplicate. Precision was calculated as 2 RSD of the three replicate values for a sample. The calculablelprecision result for sample FCB24 was 6.0 RSD, which was within the program's specified control 1 imits of \pm 20 RSD. Precision RSD was not able to be calculated for samples FC-B30 and FC-B38 because one or more of the results for each sample was less than the detection limit.

No contamination was observed in any of the preparation blanks. One blank was analyzed in each. batch of samples.

\section{Uraniun by Laser-Induced Kinetic Phosphorescence}

Samples were analyzed for uranium using procedure LA-925-009, revision A-0, on May 8, 12 and June 13, 1995. No procedural anomalies occurred during the analyses, and there were no technical difficulties. Semple results ranged from less than the detection 1 imit to $4.40 \mu \mathrm{g} / \mathrm{ml}$.

Analytical accuracy was determined on the basis of spiked samples. The spike percent recoveries ranged from 132.0 to 161.0 , where none were with in the program's specified control limits of $100 \pm 20$ percent recovery. The consistency of the positive spike bias tended to indicate that background interference was present in the samples. No attempt was made to determine or 
resolve the interference. Uranium control standards performed well, with results ranging from 92.7 to 103.1 percent recovery.

Analytical precision was evaluated on three samples, where the analyses were performed in triplicate. Precision was calculated as 2 RSD of the three replicate values for a sample. The precision results ranged from 1.5 to 11.4 RSD, which were within the program's specified control limits of \pm 20 RSD.

No contamination was observed in any of the reagent blanks. One reagent blank was analyzed in each batch of samples.

\section{Strontiun-89/90 by Separation/Proportional Counting}

Samples were analyzed for ${ }^{89 / 90} \mathrm{Sr}$ using procedure $L A-220-101$, revision $0-1$, on May 8, 11, 19 and June 12, 1995. No procedural anomal jes occurred during the analyses. There were no technical difficulties other than that two samples could not be analyzed for this analyte because of insufficient sample volume. Sample results ranged from 0.000258 to $2.36 \mu \mathrm{Ci} / \mathrm{ml}$.

Analytical accuracy was determined on the basis of spiked samples. The spike percent recoveries ranged from 93.0 to 122.3 , where two of the three spikes were within the program's specified control limits of $100 \pm 20$ percent recovery. Strontium-89/90 control standards performed wel1, with results ranging from 93.6 to 99.4 percent recovery.

Analytical precision was evaluated on three samples, where the analyses were performed in triplicate. Precision was calculated as 2 RSO of the three replicate values for a sample. The precision results ranged from 3.7 to 29.1 RSD, where two of three were within the program's specified control limits of \pm 20 RSD.

No contamination was observed in any of the reagent blanks. One reagent blank was analyzed in each batch of samples.

\section{Tritium by Lachat Micro-Distillation/Liquid Scintfliation Counting}

Samples were analyzed for tritium using procedure LA-218-114, revision A-4, on May 5, 7 and June 12, 1995. No procedural anomalies occurred during the analyses. There were no technical difficulties. Sample results ranged from 0.0000177 to $0.0307 \mu \mathrm{Ci} / \mathrm{ml}$.

Analytical accuracy was determined on the basis of spiked samples. The spike percent recoveries ranged from 86.2 to 209.1 , where only one of the three spikes was within the progran's specified control limits of $100 \pm 20$ percent recovery. From a literature review of the background radiochemical quantities sufficient to create significant bias in accuracy, the likely source of. interference was indeterminable. The actual concentrations of all other radiochemicals found in samples for this project were determined to be 


$$
\text { WHC-SD-WM-DP-138, REV. O }
$$

insufficient to cause significant accuracy bias. Tritium control standards performed wel1, with results ranging from 97.3 to 113.7 percent recovery.

Analytical precision was evaluated on three samples, where the analyses were performed in triplicate. Precision was calculated as 2 RSD of the three replicate values for a sample. The precision results ranged from 1.4 to 4.2 RSD, which were all within the program's specified control limits of \pm 20 RSD.

No contamination was observed in any of the reagent blanks. One reagent blank was analyzed in each batch of samples.

\section{Cesiun-137 by Gama Energy Analysis (GEA)}

Samples were analyzed for ${ }^{137} \mathrm{Cs}$ using procedure $L A-548-121$, revision $D-1$, on May 4 and 11, 1995. One batch of data was rejected and rerun because the ${ }_{13} \mathrm{Cs}$ control standard failed to meet laboratory operational control limits. other than the one rejected batch, no procedural anomalies occurred during the analyses. There were no technical difficulties. Sample results ranged from 0.409 to $32.7 \mu \mathrm{Ci} / \mathrm{ml}$.

Analytical accuracy was determined on the basis of a ${ }^{137} \mathrm{Cs}$ control standard. The percent recoveries of the control standard ranged from 100.0 to 101.9 , and were within the progran's specified control limits of $100 \pm 10$ percent recovery. The spiking of samples for GEA analytes is technically not possible, consequently no spiked analyses were performed.

Analytical precision was evaluated on five samples, where the analyses were performed in triplicate. Precision was calculated as 2 RSD of the three replicate values for a sample. The precision results ranged from 0.1 to 3.5 RSO, which were within the program's specified control limits of \pm 10 RSD.

No contamination was observed in any of the reagent blanks. One reagent blank was analyzed in each batch of samples.

\section{Americium-241 by GEA}

Samples were analyzed for ${ }^{241}$ Am using procedure $L A-548-121$, revision $D-1$, on May 4 and 11,1995 . No procedural anomal ies occurred during the analyses. There were no technical difficulties. All sample results were less than the detection limit, which were greater for this procedure than for analysis of ${ }^{261}$ Am by extraction/alpha energy analysis.

Analytical accuracy was determined on the basis of a surrogate $\left({ }^{137} \mathrm{Cs}\right)$ control standard. The percent recoveries of the control standard ranged from 101.5 to 101.9 , and were within the program's specified control limits of $100 \pm 10$ percent recovery. The spiking of samples for GEA analytes is technically not possible, consequently no spiked analyses were performed. 


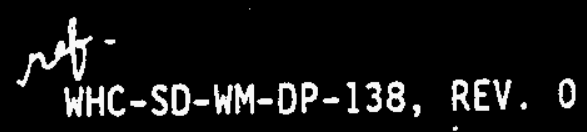

Analytical precision was evaluated on two samples, where the analyses were performed in triplicate. Precision is typically calculated as 2 RSO of the three replicate values for a sample; however, the RSD values for both of the samples was incalculable because all analytical values were less than the detection limit.

No contamination was observed in any of the reagent blanks. One.reagent blank was analyzed in each batch of samples.

\section{Americium-241 by Extraction/Alpha Energy Analys is (AEA)}

Samples were acid digested on May 2, 5, 10 and 22, 1995, using procedure LA505-158, revision A-4, and then analyzed for ${ }^{241} \mathrm{Am}$ using procedure LA-953-103, revision A-3, on May 15 and 16 and June 2, 1995. No procedural anomalies occurred during the analyses. There were no technical difficulties. All sample results were less than the detection limit.

Analytical accuracy was determined on the basis of spiked samples. The spike percent recoveries ranged from 82.0 to 94.3 , and were with in the program's specified control 1 imits of $100 \pm 20$ percent recovery. Americium-241 control standards performed within laboratory acceptance, with results ranging from 86.6 to 96.1 percent recovery.

Analytical precision was evaluated on three samples, where the analyses were performed in triplicate. Precision is typically calculated as 2 RSD of the three replicate values for a sample; however, the RSD values for each of the three samples was incalculable because all analytical values were less than the detection limit.

No contamination was observed in any of the preparation blanks. One preparation blank was analyzed in each batch of samples.

\section{Plutonium-239/240 by Ion Exchange/AEA}

Samples were acid digested on May 2, 5, 10, and 22, 1995, using procedure LA505-158, revision A-4. The analys is of 239/240 pu was performed using two procedures: $L A-503-156$, revision $A-0$, and $L A-943-127$, revision $A-0$, on May 17,24 and 25 and June 5, 1995. The program specified the use of the former procedure; however the laboratory discontinued its use midway through the project and replaced it with the latter procedure. Sample FC-B40 was rerun because its AEA resolution was poor. Other than the one sample which was rerun, no procedural anomalies occurred during the analyses. There were no technical difficulties. Except for sample FC-845, which had a result slightly greater than the detection limit, all sample results were less than the detection limit.

Analytical accuracy was determined on the basis of spiked samples. The spike percent recoveries ranged from 75.6 to 96.9 . Two of the three spikes were 
$\rho H C-50-W M-D P-138$, REV. 0

within the program's specified control limits of $100 \pm 20$ percent recovery. For those data cited in this report, the performance of $239 / 240 \mathrm{Pu}$ control standards was within laboratory acceptance limits, with results ranging from 83.6 to 100.0 percent recovery.

Analytical precision was evaluated on three samples, where the analyses were performed in triplicate. Precision is typically calculated as 2 RSD of the three replicate values for a sample; however, the RSD values for each of the three samples was incalculable because two or more of the analytical values for each sample were less than the detection limit.

No contamination was observed in any of the preparation blanks. One preparation blank was analyzed in each batch of samples.

\section{Plutonium-238 by Ion Exchange/AEA}

Samples were acid digested on May 2, 5, 10 and 22, 1995, using procedure LA505-158, revision A-4. The analys is of ${ }^{238} \mathrm{Pu}$ was performed using two procedures: LA-503-156, revision A-0, and LA-943-127, revision A-0, on May 17, 24 and 25 and June 5, 1995. The program specified the use of the former procedure; however the laboratory discontinued its use midway through the project and replaced it with the latter procedure. Sample FC-840 was rerun because its AEA resolution was poor. Other than the one sample which was rerun, no procedural anomalies occurred during the analyses. There were no technical difficulties. All sample results were less than the detection limit.

No control standard exists for ${ }^{238} \mathrm{Pu}$, consequently it was not possible to spike samples to evaluate analytical accuracy. Because ${ }^{238} \mathrm{Pu}$ is a byproduct of $239 / 240 \mathrm{Pu}$ analysis, it is possible to use the ${ }^{239} \mathrm{Pu}$ control standard as a surrogate for evaluation of ${ }^{238} \mathrm{Pu}$ accuracy. The performance of ${ }^{239} \mathrm{Pu}$ control standards was within laboratory acceptance limits, with results ranging from 83.6 to 100.0 percent recovery.

Analytical precision was evaluated on threa.samples, where the analyses were performed in triplicate. Precision is typically calculated as 2 RSO of the three replicate values for a sample; however, the RSO values for each of the three samples was incalculable because all. of the analytical values for each sample were less than the detection limit.

No contamination was observed in any of the preparation blanks. One preparation blank was analyzed in each batch of samples. 
WHC-SD-WH-DP-138, REV. O 
WHC-SD-SNF-DP-003, Rev. 0
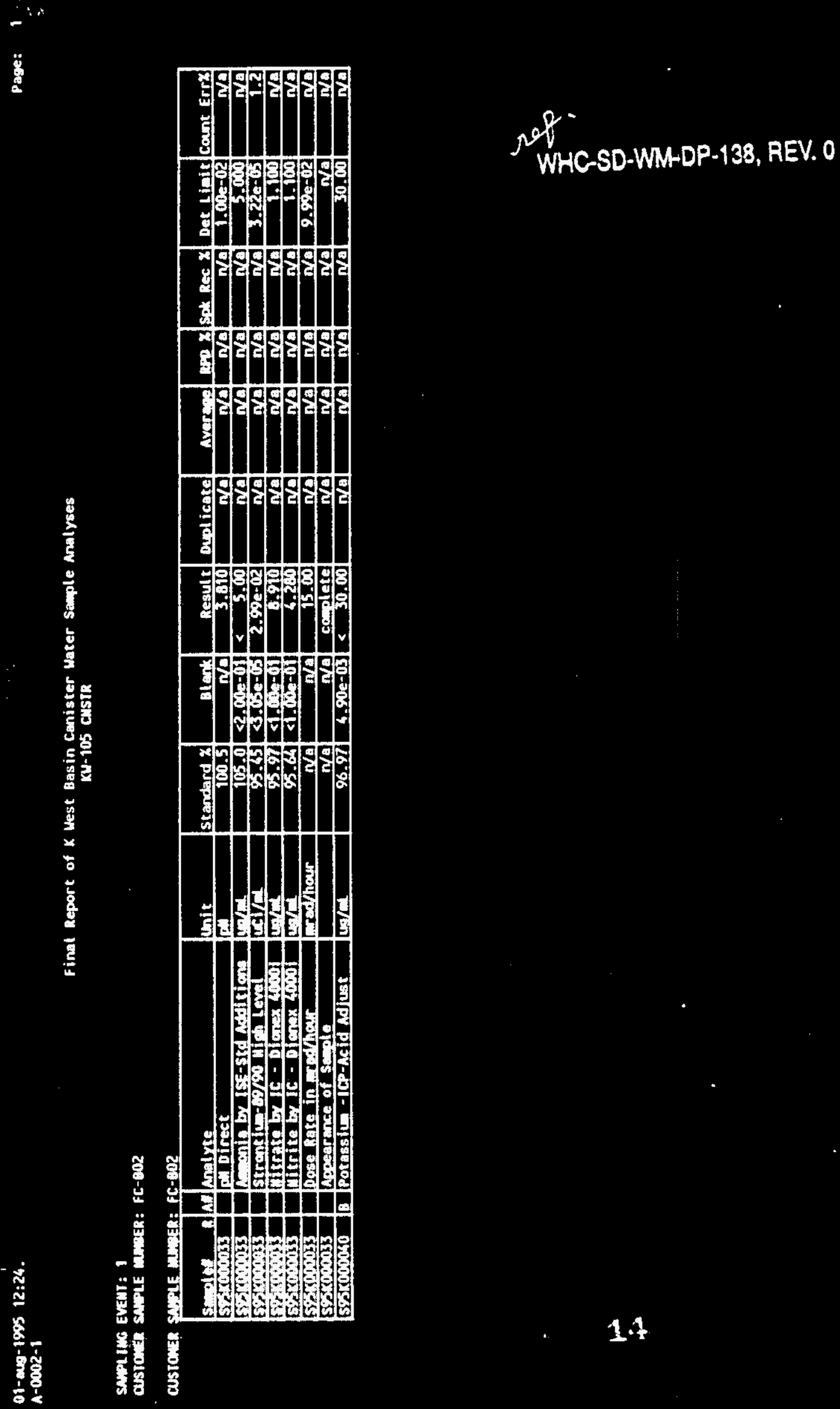
WHC-SD-SNF-DP-003, Rev. 0

N

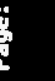

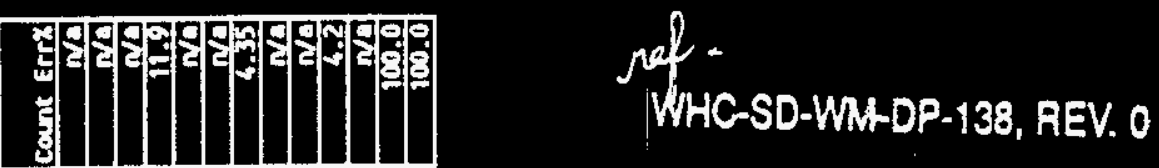

递

gen

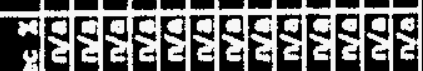

tै

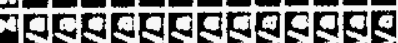

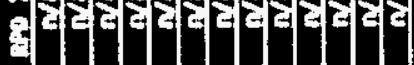

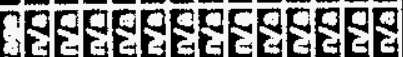

y

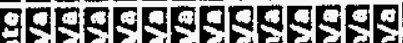

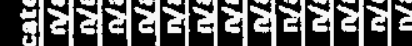

है

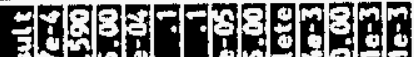

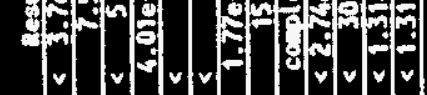

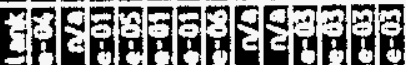

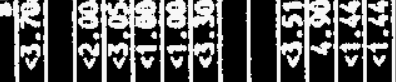

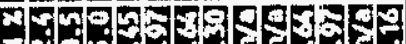

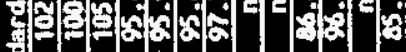

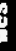

部

15

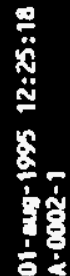

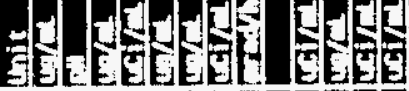

is

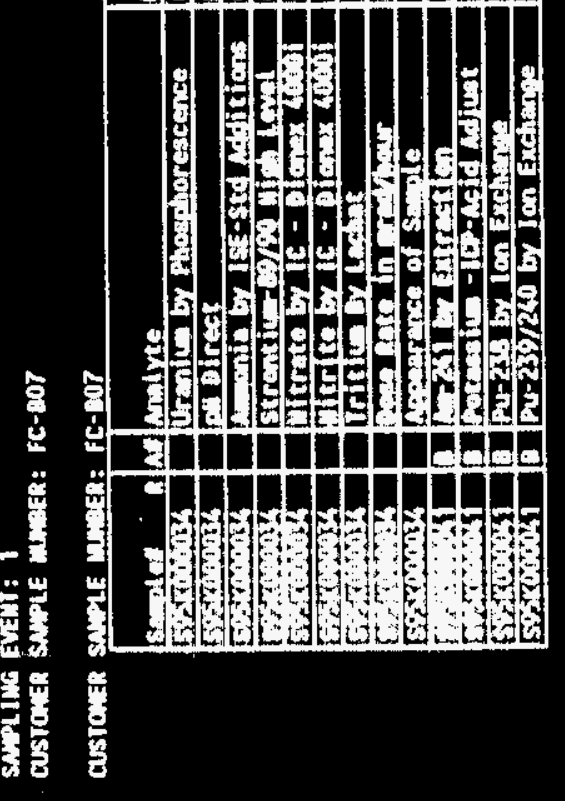


WHC-SD-SWF-DP-003, Rev. 0

$m$

sof -

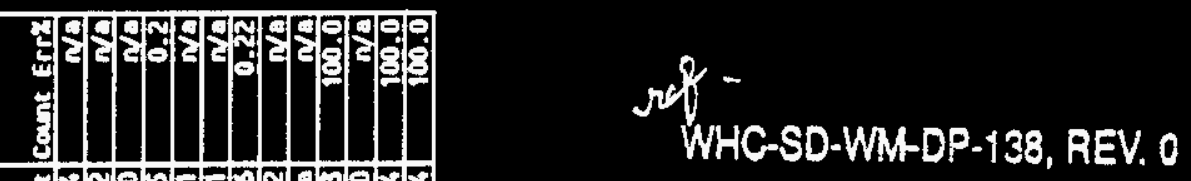

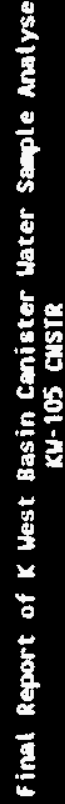

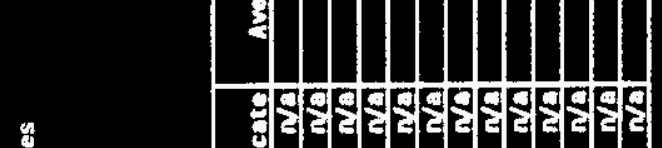

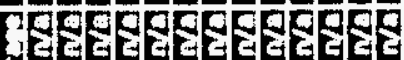

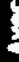

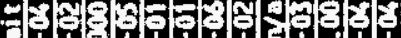

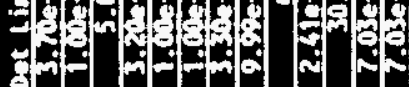

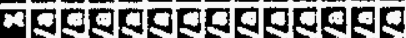

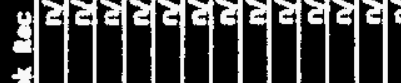

pagadagagage

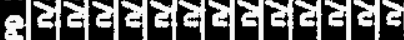

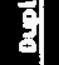

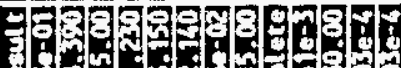
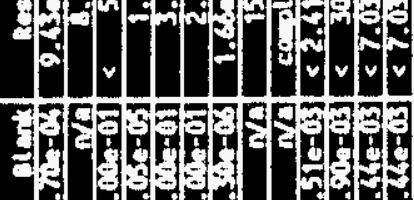

-

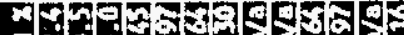

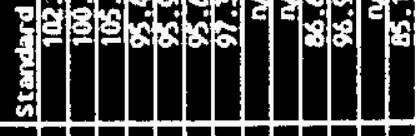

H $d \leq d \leq d$

SEEEEED CES

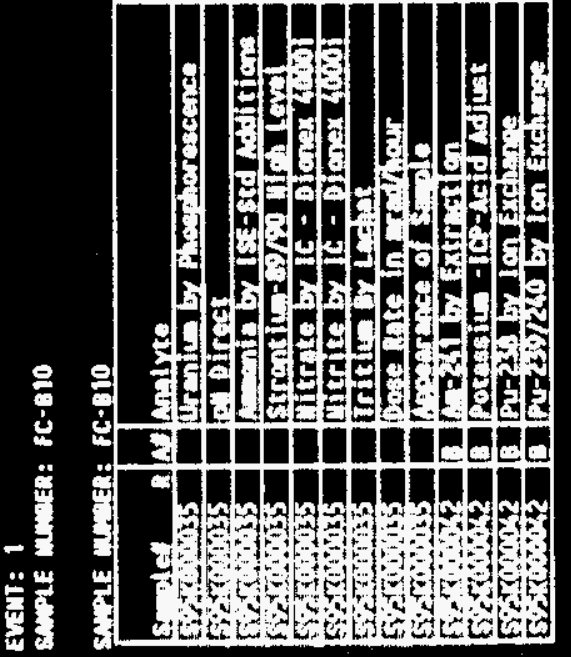

16

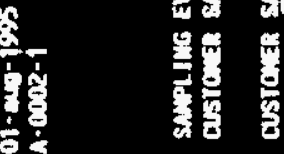


WHC-SD-SNF-DP-003, Rev. 0

i

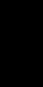

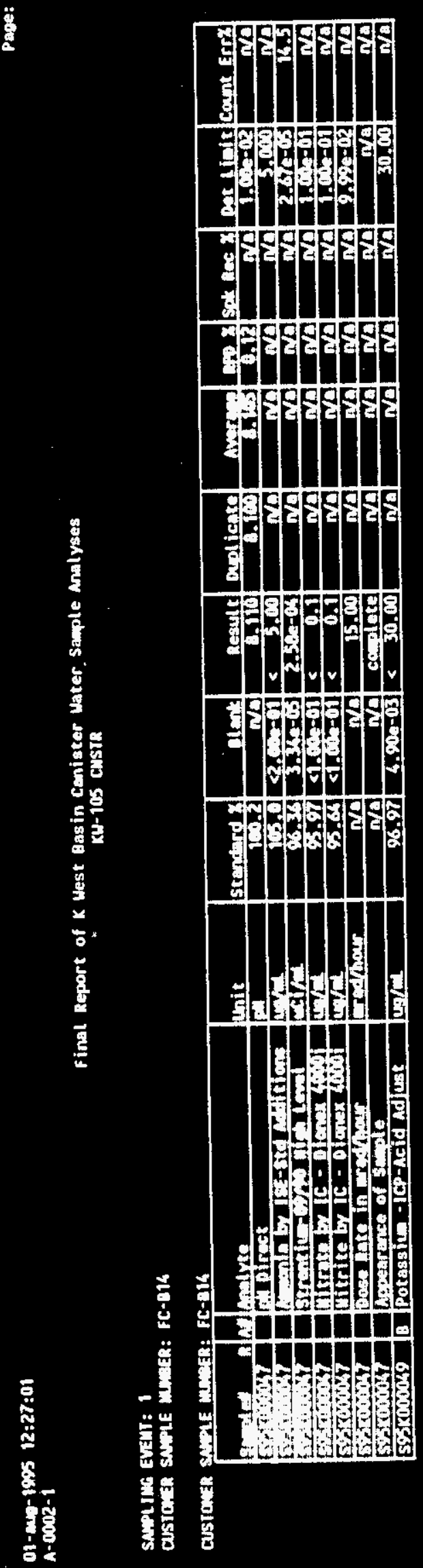

nef.

WHC-SD-WM-DP-138, REV. 0

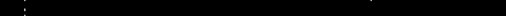


WHC-SD-SNF-DP-003, Rev. 0

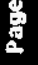
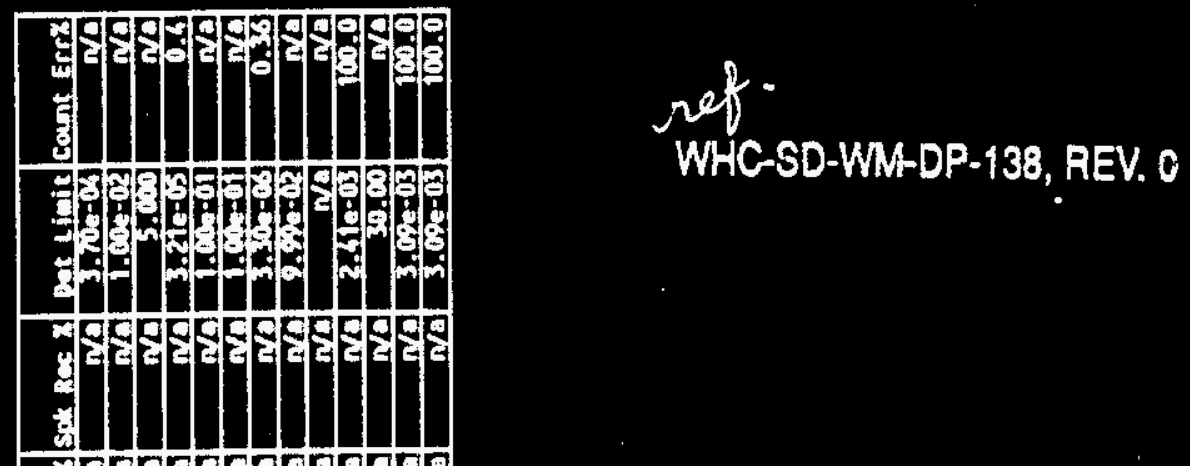

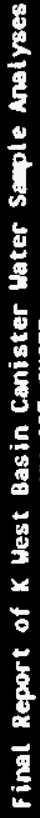

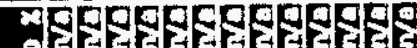

각

Bagagagegar
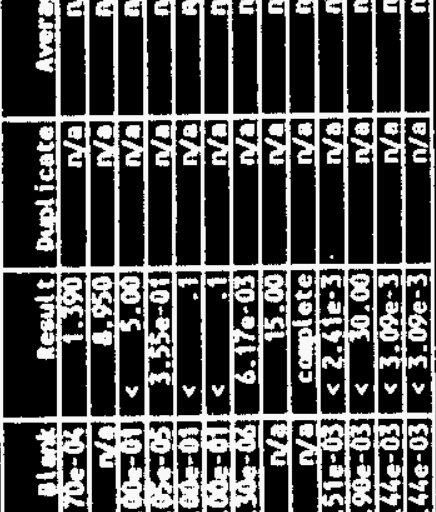

A $\Rightarrow$

(v) vrvar $\forall \Rightarrow 7$
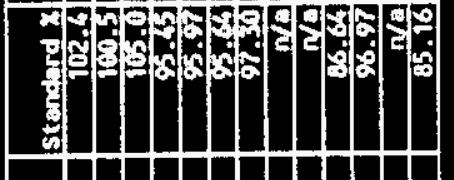

要

$\Rightarrow d \mathrm{~d} d \mathrm{~d}$ - $\mathrm{d} d \mathrm{~d}$

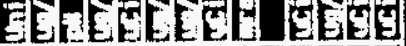

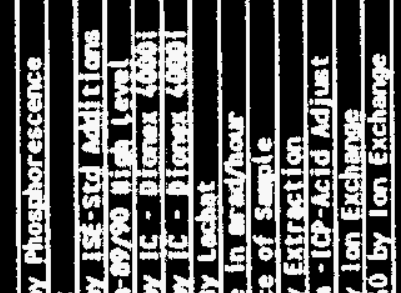

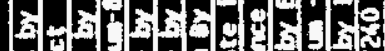

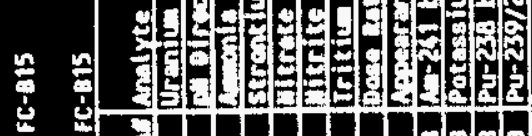

i.

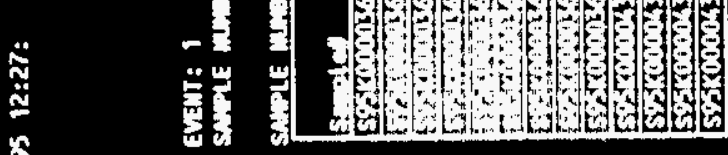

E $\quad 1 \%$ 
WHC-SD-SNF-DP-003, Rev. 0

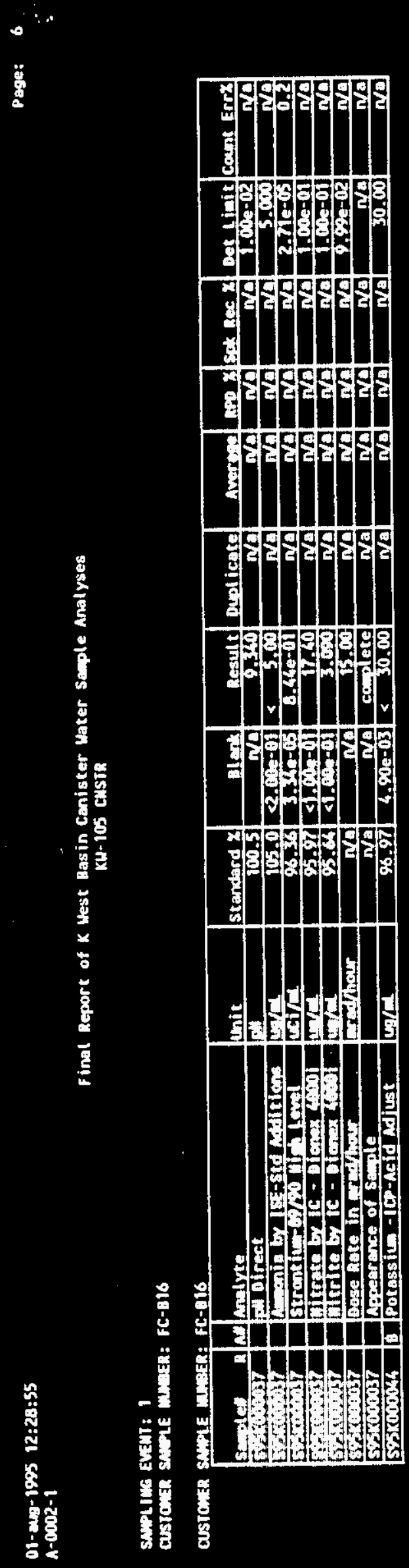

ref-

\section{9}


Final Report of $\mathrm{K}$ Mest Basin Canister Mater Sample Mnatyses KU- 105 custh

SMPL ING EVEAT: 1

custaned smble mpekt FC-B17

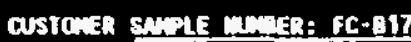

\begin{tabular}{|c|c|c|c|c|c|c|c|c|c|c|c|}
\hline State: & anlese & thit & Standand $\mathbf{x}$ & Arert & & Drodicate & & & & & Count Erry \\
\hline 52,51013 & PDirect & 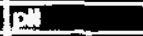 & 100.5 & $\mathrm{na}$ & 8.25 & and & $n$ & na & Ia & & $\mathrm{nD}$ \\
\hline $2 \pi, 3$ & 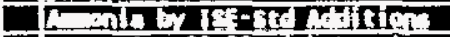 & & $\mathrm{TH}$ & [n]-11 & 5.0 & na & n & In & na & & $n$ \\
\hline Eesing & 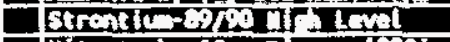 & CIII & 96.3 & $3.3 e-13$ & ins.s? & ne & 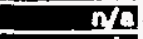 & na & no & & Da \\
\hline $22 \pi+1$ & Dirrite by ic - Diede $\mathrm{Wh}$ & & Q5.97 & s.t., 10 & $2.710-41$ & $\mathbf{n} \mathbf{0}$ & $n$ & $n$ & $n$ & & $n$ \\
\hline 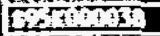 & 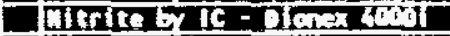 & $m C$ & 9.64 & $21.00=01$ & & na & ne & $\mathbf{n a}$ & nat & & na \\
\hline 50013 & Desectite in & Extran & $n / a$ & $\mathrm{n/0}$ & -15.00 & 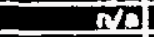 & n' & ID & na & Q. & $\mathbf{n} \mathbf{D}$ \\
\hline 50200030 & Livearrence of a ple & & no & & ceplete & na & ne & no & nza & & na \\
\hline $5 \% 5006045$ & 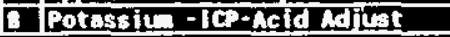 & 배르. & 96.97 & $4.010-03$ & $<30.00$ & a/a & & $n$ & in & 30.00 & $n / a$ \\
\hline
\end{tabular}

)

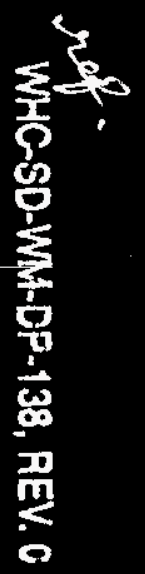


SNPLINS EXENT: 1

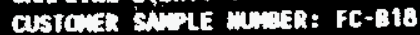

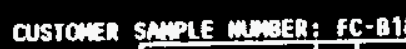

\begin{tabular}{|c|c|c|c|c|c|c|c|c|c|c|c|}
\hline . & & & & & & & & & & & \\
\hline siple: & Wualyte & Lnit & Stendard $\mathrm{y}$ & Alnts & Bente & Diplicales & & 23 & $\frac{25}{28}$ & $\mathrm{I}$ & $\frac{\operatorname{lng}}{n / 8}$ \\
\hline 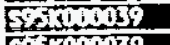 & Poireat & PI & 109.5 & $\frac{120}{2+3-01}$ & $\frac{9.160}{5.00}$ & $\frac{1 y}{90}$ & $\frac{129}{n 20}$ & $\frac{120}{n / 2}$ & $n 20$ & 5.00 & $\frac{140}{n ! a}$ \\
\hline E Edo & Eanie by IEs-std aditions & $\cos / 2$ & $\frac{112.4}{9.34}$ & 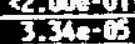 & Intse. & $\frac{\operatorname{lng}}{\mathrm{n} 20}$ & a & $\mathbf{A Q}$ & $n a$ & $1.0 .02-10$ & $n a$ \\
\hline 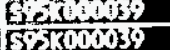 & & $\frac{102}{4}$ & 95.97 & रा. & 25.5 & $\mathbf{n a}$ & No & Ix & ne. & $1,0,2-01$ & $n / 2$ \\
\hline 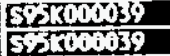 & 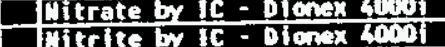 & 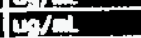 & 95.64 & $<1.01,-01$ & $7.72 \mathrm{e}-01$ & $n / 0$ & n/a & na & ne & $1.000-01$ & ala \\
\hline & 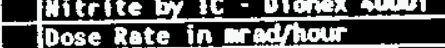 & Erethour & $\sqrt{2}$ & 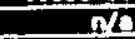 & 15.02 & no & na & $n$ & n/a & 9.2ie-12 & n!e \\
\hline 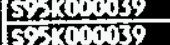 & flose core in longle & & $n / 0$ & $n / a$ & canplete & $n / s$ & ns & $\mathrm{m}$ & $\mathrm{D} / \mathrm{C}$ & $n / 9$ & ngs \\
\hline 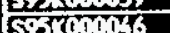 & Potassitn-ICP-Acid adust & $\cot / \mathrm{Cl}_{2}$ & 96.97 & $4.90-03$ & $\leq 3.00$ & & n/2 & $n$ & $n<0$ & & \\
\hline
\end{tabular}

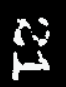


SMiptins EVEUT: 1

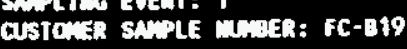

CUSTOMER SWRLE WMER: FC-819

\begin{tabular}{|c|c|c|c|c|c|c|c|c|c|c|c|}
\hline "mate"t & & & Standared $x$ & & & Punl icate & Avereme & Det $x$ & & & \\
\hline 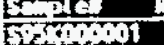 & allanalye & 91 & 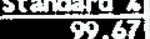 & & $\frac{109010}{80}$ & na & ing & No & $\frac{22}{n / 0}$ & $1.015=-12$ & $\frac{100}{n / 0}$ \\
\hline 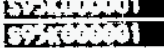 & Durect & $\mathrm{E}$ & $\frac{2.04}{92.01}$ & 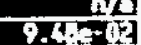 & $<|| 1+7\})$. & $\frac{2}{a}$ & 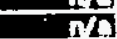 & $n$ & no & 10.0 & $n$ \\
\hline Whin & 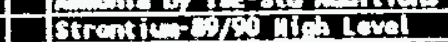 & are & 98.10 & $\langle 3.62=-4$ & 1.1 .0 & nia & no & no & na & 5.0.2- & $1.9 x+0$ \\
\hline $\operatorname{sen}+1$ & 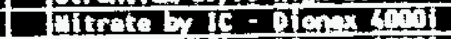 & ETC & क.2. & 4. [0.1] & 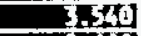 & $n$ & na & 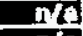 & $n$ & 2.00 & n/2 \\
\hline $2=1$ & 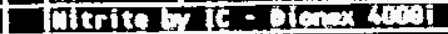 & $2+2$ & 9.17 & 8.1 .0 & 2.63 & na & $n e$ & $\mathbf{i} / \mathrm{a}$ & na & & ng \\
\hline ERTin & Costic-ily brex & rant & 10.0 & 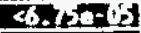 & 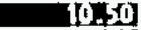 & $\mathbf{m a}$ & ID & $n$ & N & 6.1.20-62 & 0.26 \\
\hline EDSTCO & Doce hate in oredpons & Erefiour & n/8 & $\mathrm{m} / \mathrm{B}$ & 15.0 & n/a & ng & $n / 0$ & $n=$ & $9.8 \mathrm{se}-02$ & 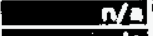 \\
\hline Sescong & Acpearance of stale & & n/a & $n / 9$ & COWPLEIE & n/a & na & 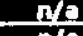 & n/a & $\frac{a / 2}{2 n}$ & n/a \\
\hline & -sein-rce-Acid & W/ & .40 & $4.48 \mathrm{e}-02$ & 30.00 & $n$ & $n / 0$ & $\mathbf{n} / \mathbf{a}$ & 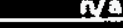 & SOS.W & $\mathbf{n} \mathbf{a}$ \\
\hline
\end{tabular}

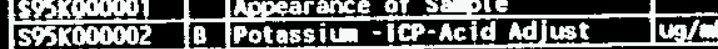

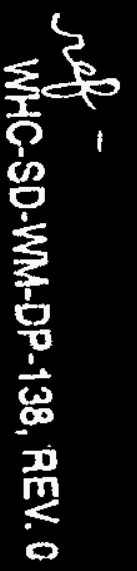


Final Report of $K$ West Basin Cenister Water Saple Analyses KU-105 CHsTR

SAPLING EVET: 1

CUSTCES SMPLE MUMER: FC-421

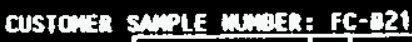

\begin{tabular}{|c|c|c|c|c|c|c|c|c|c|c|c|}
\hline smole* & Mlanive & Git & stendard $x$ & Etrsts & Result & molicate & $r$ & $m x$ & $\cos x$ & Det lipit & Count Erry \\
\hline $5 \cdot \sin 1$ & 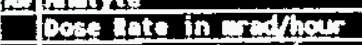 & crenthorr & $n / a$ & no & 15.00 & 50 & n/o & $n$ & no & $9.920-02$ & $n / 9$ \\
\hline Sostions & Crosarouse of $\mathrm{s}$ ole & & no & $\overline{m a}$ & coplete & me & na & $n \mathbf{a}$ & na & $n / a$ & $\mathbf{n} \mathbf{0}$ \\
\hline
\end{tabular}

衿

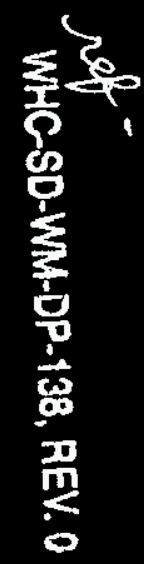


SMPLIUG EVEMT: 1

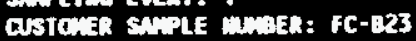

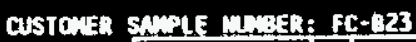

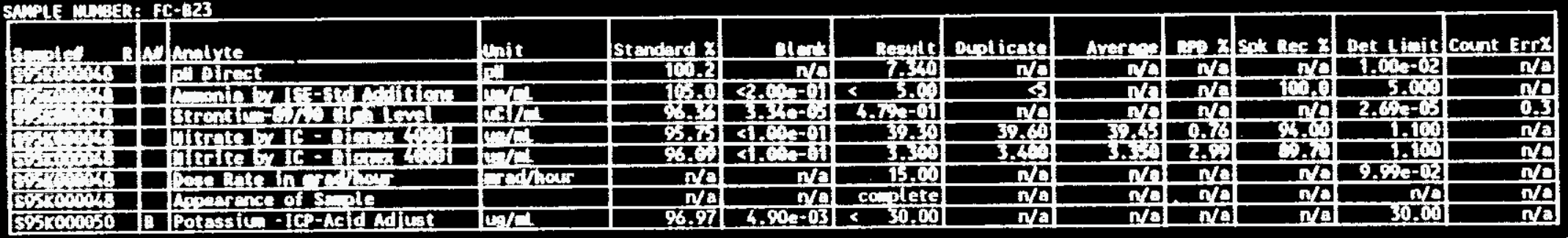

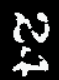

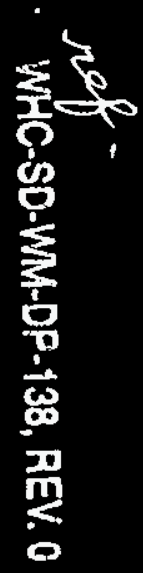


WHC-SD-SNF-DP-003, Rev. 0

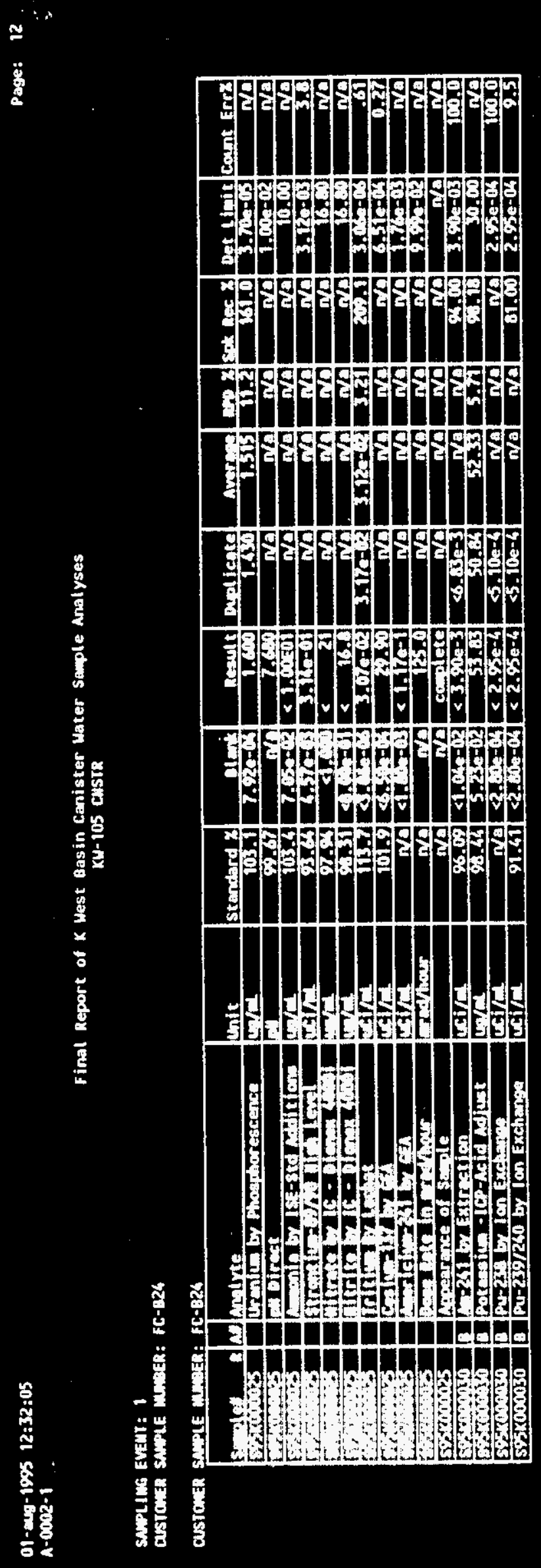




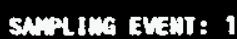

custowit swite where: FC-825

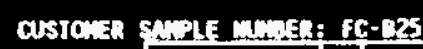

\begin{tabular}{|c|c|c|c|c|c|c|c|c|c|c|c|}
\hline (5. & Intre & thit & Stentard $x$ & Ilm! & Aresult & paplicate & AMAR & & & & Cont Erex \\
\hline Lithos & Pirect & Fin & 6.67 & $m$ & 7.0 .1 & in & $n a$ & $n \pi$ & $n a$ & $1.65-12$ & Na \\
\hline Fe 18 & ning & 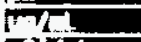 & $2+11$ & & SI & a 2 & $n$ & $n$ & na & 10.69 & $n 10$ \\
\hline Finotio & 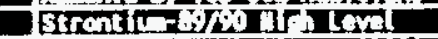 & iard & W.1. & 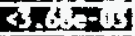 & $5.4 i e-01$ & $\mathbf{n} \mathbf{a}$ & $\mathrm{n} / \mathrm{a}$ & na & $a_{2}$ & & $2.70+0$ \\
\hline & nrrongte-arestat & & [01.2] & 4.E.t & 45.40 & a & $n$ & $n$ & Ma & 21.01 & $\mathbf{n}$ \\
\hline Fin & 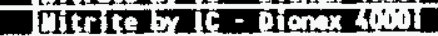 & $E$ & 1000 & S.6. & 16.0 & na & $\mathbf{n}$ & na & $\mathrm{m} 2$ & {$[\mathrm{C.} . \mathrm{J}$} & 20 \\
\hline Fit? & Cotit $=1\}$ b $P A$ & an & 1100 & Shits= & 1,230 & nta & 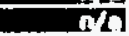 & $n 2 a$ & n & Q14 & $0,6]$ \\
\hline Eichis & 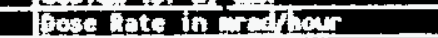 & Dhor & n/a & $n / 0$ & 40.03 & nia & na & D & 12 & Q.ingen & $\mathrm{na}$ \\
\hline Fre $104+15$ & Traprace of $a$ or & & n/a & $\mathbf{n} \mathbf{a}$ & coulets & n/a & Ma & $n$ & n/o & $n / 2$ & n \\
\hline Sisko00017 & Potassic-ICP-Acid NdTust & 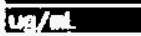 & 95.60 & $6.4 .8 \cdot 02$ & 35.0 & $\mathbf{n a}$ & $\mathrm{na}$ & no & 12.4 & 30.00 & na \\
\hline
\end{tabular}

i

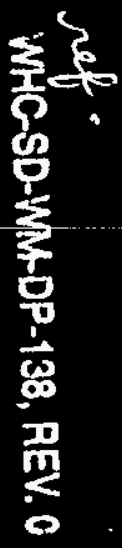


WHC-SD-SNF-DP-003, Rev. 0

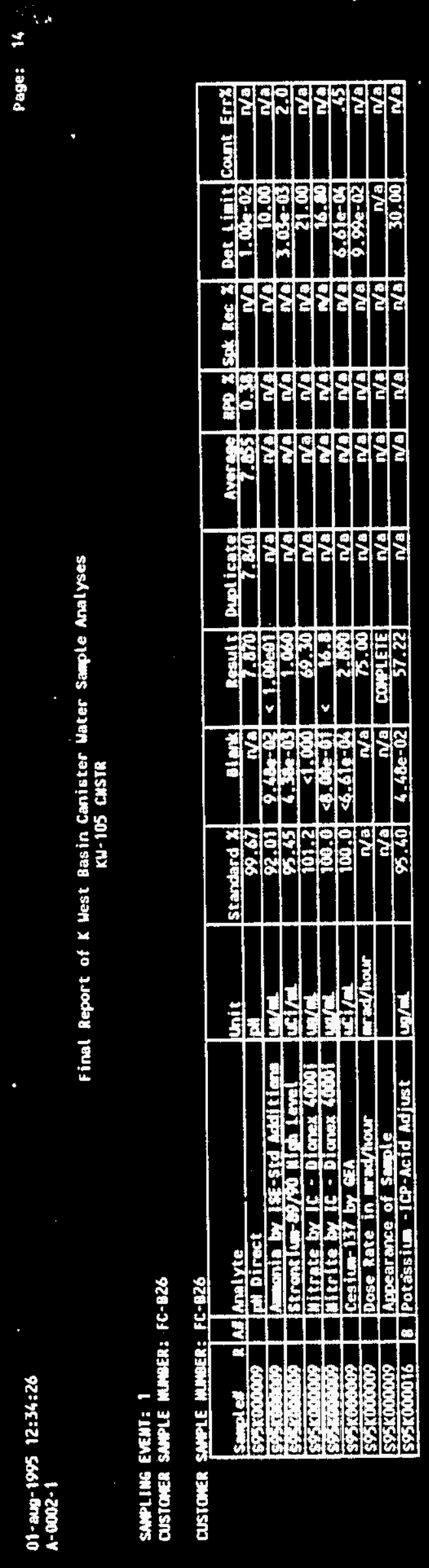


WHC-SD-SWF-DP-003, Rev. 0

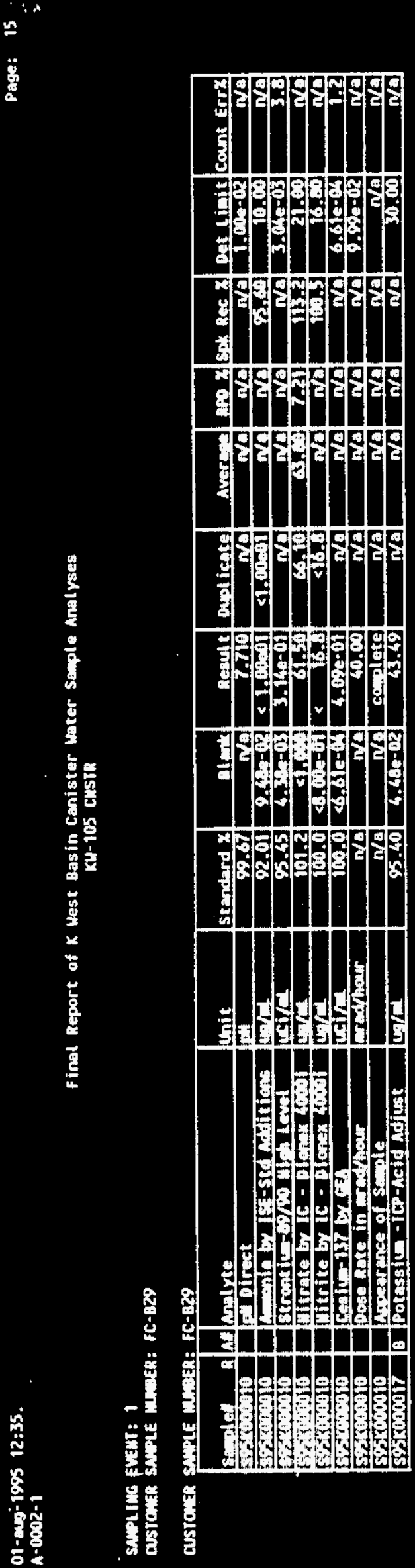

nef -
WHC-SD-WASDP-133, REV. 0 
SAmpting EVEAT: 1

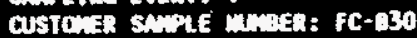

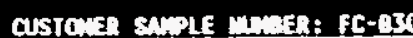

\begin{tabular}{|c|c|c|c|c|c|c|c|c|c|c|c|}
\hline$\pi$ & Anite & & Stmond $\mathrm{z}$ & & & aplicats & & & & & as Erry \\
\hline & atrescese & m & 92.63 & $3 . \pi \cdot 0$ & 1,5 & -1.5 & IE: & & & & na \\
\hline 4 & Plfirat & & and & ${ }_{n}^{2} n$ & 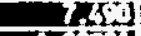 & $n=$ & 2 & $\mathbf{n} \boldsymbol{n}$ & $n D$ & & $m a$ \\
\hline 4 & 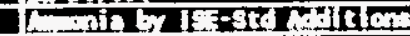 & & Iixn & 7. $0 . \ln ^{-1}$ & C.1. 3.1 & na & $m 2$ & DD & 20 & 4 & 92 \\
\hline$F^{\prime} T$ & 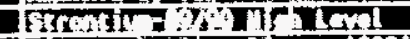 & Eतर & Fing & 4x+e-n] & 2.39 & $\mathbf{m} \mathbf{3}$ & $\mathrm{M} 2$ & $\sqrt{2}$ & $\mathbf{n a}$ & $2.910-13$ & 1.3 \\
\hline 603 & liments & {$[+2$} & 90 & SW. & 21 & na & 토을 & $\mathbf{n} \mathbf{2}$ & $\mathbf{m o}$ & 2100 & an \\
\hline$E$ & 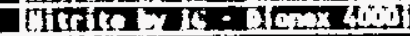 & E. & Yisil & C.[its-ul] & 6.9 & na & $R / 9$ & $8^{2}$ & $n / 2$ & 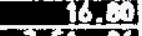 & na \\
\hline & Intris $x$ & Cali & Ms.? & 《eseot & 2.5 & $2 . \operatorname{sen} 1$ & 2. $\log _{-1}$ & 10 & [ie亚] & 3.51e-13 & \\
\hline 5,78 & Pits & Min! & IIS] & 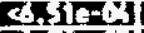 & 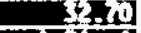 & [S":3] & SB, & Fin & $n / a$ & G.5se-63 & 0.23 \\
\hline $2=5$ & $\Rightarrow$ Tre & $T=\sqrt{c}$ & no & S.I. & $\leq 1.2 \mathrm{n}-1$ & 41.201 & na & as & $\mathbf{E} \boldsymbol{c}$ & & $\mathrm{na}$ \\
\hline 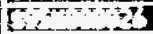 & rorir & Exiser & na & $n a$ & $1 \mathrm{bog}$ & $\mathbf{n a}$ & no & an & $n$ & Q 1 is $=-13$ & na \\
\hline$[2 \pi 53$ & 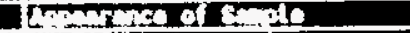 & & ang & $\mathbf{n}$ & covese & na & $n$ & Nal & 124 & $a / a$ & $n / 8$ \\
\hline 071 & 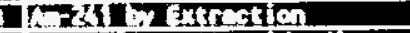 & EII & 91.13 & {$[0-6]$} & $<3 \times 50=3$ & S.5ne-1 & $n$ & $n$ & & 3. $3 e-0 \mathrm{~s}$ & 100 \\
\hline$(2,7+n$ & 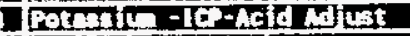 & $c^{m}$ & S.6.4. & & & $S 0.0$ & na & ne & IUI. & 3.1 .10 & n/a \\
\hline Fin & 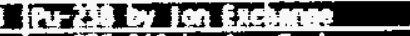 & PI/E & ng & 4..83-13 & 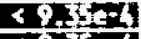 & s.11:-4 & $\mathrm{n}$ & $n$ & 99 & $9,30-4$ & 10.0 \\
\hline G.5.Tis & 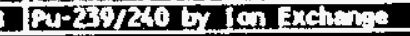 & Calle & 10.0 & 6.36 & $<9.350-4$ & st.Ile=4 & $n / a$ & $\mathbf{n} \mathbf{a}$ & \%.6. & $9.35 \mathrm{e}-04$ & 160.0 \\
\hline
\end{tabular}

溇

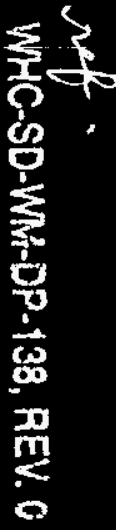

$\overline{\$}$ 
swotiug EVET: 1

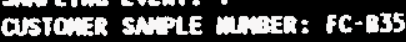

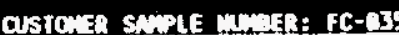

\begin{tabular}{|c|c|c|c|c|c|c|c|c|c|c|c|}
\hline 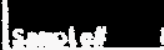 & Wanlyte & Unit & andard $x$ & $01 \mathrm{~m}$ t & Result & Molicers & Antres & $\sin x$ & set & Mint & Count Err: \\
\hline 24 & Dinst & F & \%.9. & na & 7.40 & $\mathrm{na}$ & in & $\pi$ & & & ma \\
\hline II & & & 103 & & $<1,[1]$ & S1.6301 & nh & Na & E: & Q.63 & ng \\
\hline$\therefore 8$ & tires & LE & Gili: & & [.1.1] & ne & ad & $E R$ & & 4.51e-13] & 2.0 \\
\hline & & & gin: & & $<$ & \&11 & $n$ & $n$ & & & $\mathrm{Me}$ \\
\hline$=\frac{11}{11}$ & 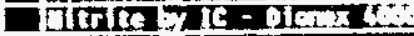 & & (3) & 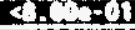 & 160. & 46.1 & $n^{2} 2$ & nL & Q26. & & no \\
\hline 611 & 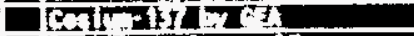 & rate & IL.6 & 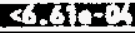 & 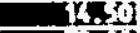 & $\mathrm{m}$ & $n h$ & A2 & $n^{2} 0$ & $3.10=1$ & .2 \\
\hline 0 iI & 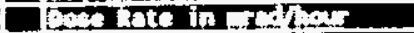 & Gorator & $n 2$ & $n 2$ & (B.3.0] & na & ag & na & $\mathbf{a} / \mathbf{e}$ & Q.98-121 & ala \\
\hline ENTiकan & Depsarene of sine & & nas & $n$ & caplets & n!a & $n a$ & $n L$ & $\mathbf{n} \mathbf{a}$ & ris & and \\
\hline S. TOUTIS & 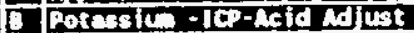 & Ex/c & 9... & $3.926-0$ & $\leq \quad 30.00$ & n/a & & & & & $n$ \\
\hline
\end{tabular}

$\stackrel{\dot{\omega}}{\sigma}$

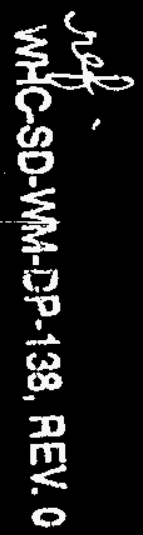


Final Report of $K$ Hest Basin Canister Hater Saple Mnalyses $\mathrm{KH}-105$ CusTR

SMPLITE EVTH:

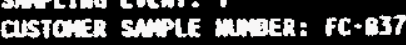

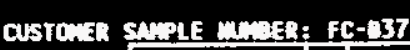

\begin{tabular}{|c|c|c|c|c|c|c|c|c|c|c|c|}
\hline .19 & Cnalyte & thit & Standard $x$ & Alms & Remalt & orplicente & Avere. & & & & ent Eert \\
\hline Fit & IDireal & Fin & 9.67 & na & 7.510 & $n a$ & $n 2$ & Wh & no & & na \\
\hline & Iris & 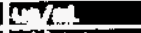 & Q.2.1] & $9 x^{x}=y^{y}$ & $<1,1]$ & $-n$ & $m T$ & 72 & $n 2$ & 10.91 & $\mathrm{n} 2$ \\
\hline$-4 T$ & Eirontits & [1] & S.45] & 4.5.5-1 & & $1.2[3]$ & 1.L1) & & 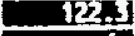 & & 2.1 \\
\hline F.77, & ffrres & QRL & 93.20 & {$\left[1,\left[t_{1}^{\top}\right]\right.$} & & $n$ & nh & $n / 2$ & $\mathrm{~s} / \mathrm{a}$ & 2.010 & $\mathbf{n} / \mathbf{a}$ \\
\hline $\mathrm{EH}+\mathrm{IP}$ & Drtimbals-actas & 12 & S.123 & $<1 .: 1$ & $\leq$ & ng & 20 & No & $n$ & & $\mathbf{n} \mathbf{9}$ \\
\hline & conthises & CAI & {$[0,0.0$} & $<6,618+2$ & D.OII] & G.0in] & 8.0.0 & Q.e: & $n / 2$ & $6.610=4$ & .27 \\
\hline Win & Destote in Crerers & Extisen & $n a$ & $n$ & $\mathbf{1 6 0 . 0}$ & $\mathrm{n} / \mathrm{a}$ & $n$ & eco & $n 2$ & $\operatorname{sen}=-6$ & $a 0$ \\
\hline G5t:IIF & Domere of chis & & ne & & co dete & n20 & $n z$ & na & $\mathbf{n}$ & $n g$ & n!o \\
\hline 0370039 & Potassion-ICp-ncid adlust & 미도 & 94.80 & $3.92 e-103$ & 0.01 & $\mathrm{n} / \mathrm{a}$ & $\mathbf{n a}$ & nes & ma & 30.01 & $\mathrm{ma}$ \\
\hline
\end{tabular}

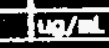

$94.8013 .92 e^{-12}<30.00$

a d $n / a$

iacm

na

$\omega$

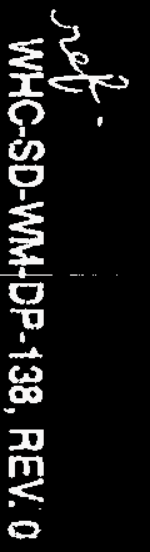


Final Report of $K$ Hest Basin Canister Mater Sample Analyses

$$
\text { kH-105 CUSTR }
$$

Surting EVEUt: 1

astowid swple chent: fC-838

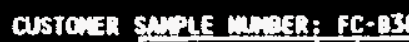

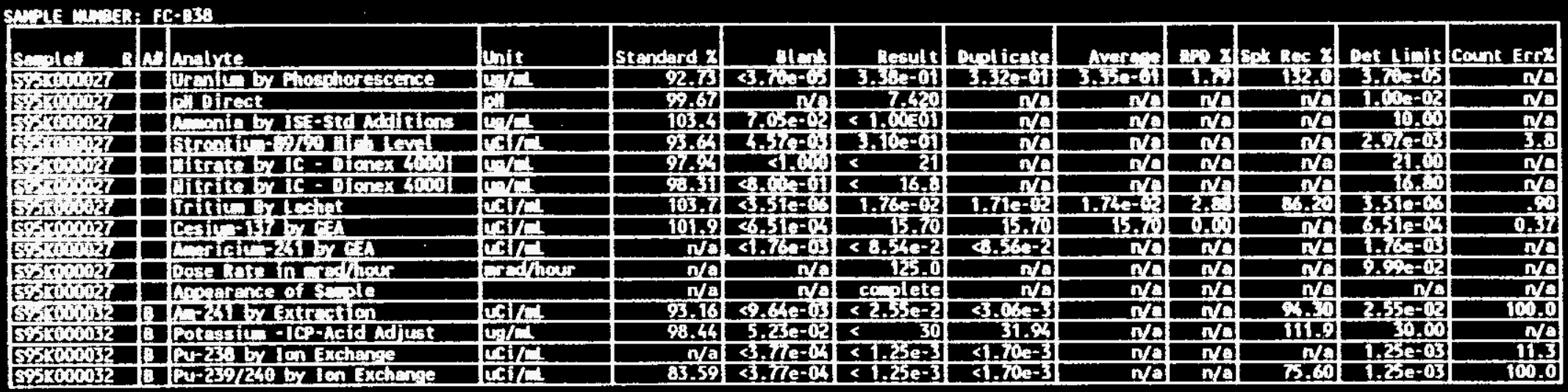

:

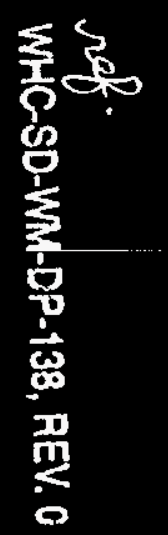


WHC-SD-SNF-DP-003, Rev. 0

$\ddot{8}$

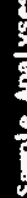

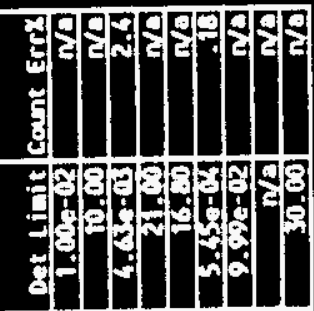

ref.

WHC-SD-WM-DP-138, AEV. 0

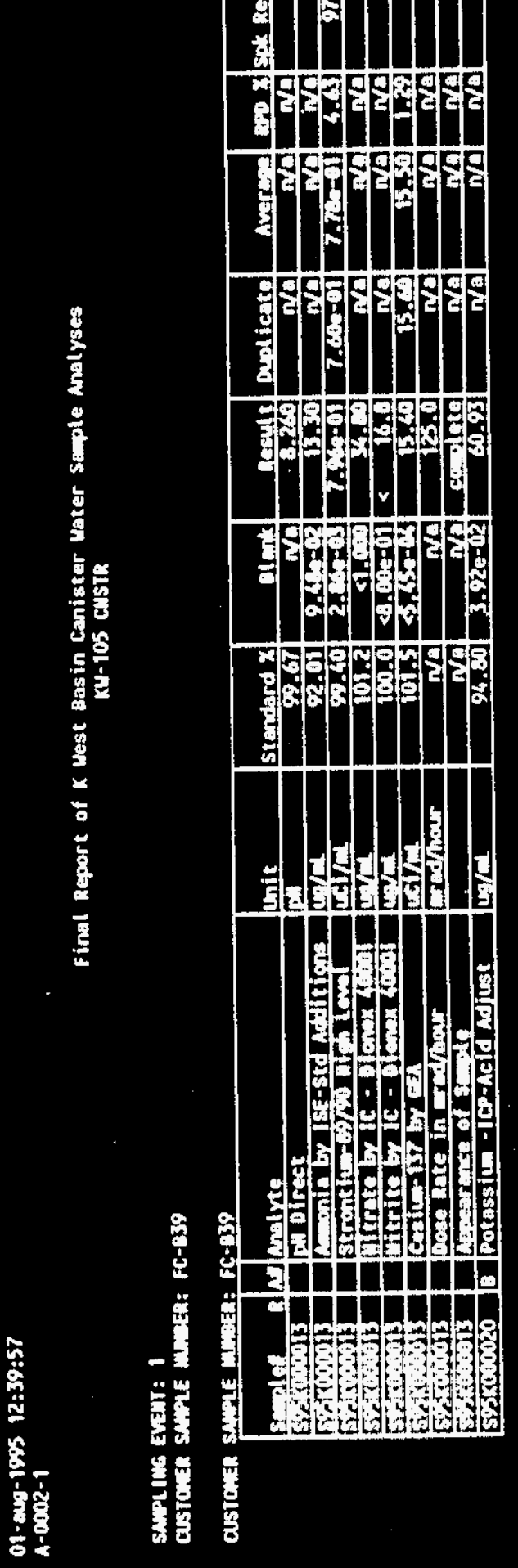

$3: ?$ 
SMPLIMS EVEAT: 1

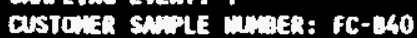

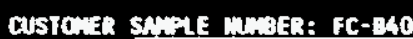

\begin{tabular}{|c|c|c|c|c|c|c|c|c|c|c|c|}
\hline 73 & Ilontre & $\ln$ & Stentind $x$ & Im: & Ecauls & ardicate & Ameres & & & & Count Err: \\
\hline Eim & 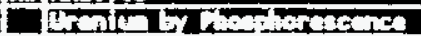 & & 103.1 & The & 4.5 & $\mathrm{no}$ & $\pi$ & $\mathrm{ac}$ & no & 3 & $n / 0$ \\
\hline$=04$ & Filreat & & घ.6.ji & $n^{M}$ & {$[5]$} & $\mathbf{m} \mathbf{a}$ & $n$ & $m$ & $\boldsymbol{n}$ & [12. & $n$ \\
\hline 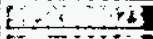 & andr-Che & & 105.1 & & CI. & $\mathrm{mo}$ & $\mathrm{no}$ & 27 & $\mathrm{mD}$ & & a \\
\hline$\because \vdots$ & Mrtite & & Q.5.5. & $\mathbf{7}$ & I. $r s=01$ & 52 & No & II & 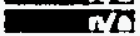 & & 5.1. \\
\hline \pm & arreta & & 6r.5 & & $<4$ & 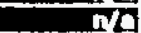 & $\mathrm{FO}$ & 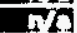 & as & & $n z$ \\
\hline & 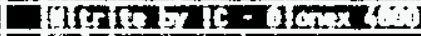 & & sing & C. & $\therefore \quad 16.5$ & na & Ih & $n$ & $n^{2}$ & & na \\
\hline$\therefore$ & Erite alest & 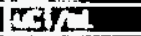 & TIF. & & $2.16-12$ & $n$ & $n$ & $n$ & 90 & & .41 \\
\hline Fientist & 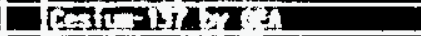 & Tan & THE & $2 \pi+1=1$ & 21.40 & na & 52 & $\sqrt{2}$ & $\mathrm{no}$ & & .15 \\
\hline Licisis & $L \operatorname{ran} 2102 \pi$ & 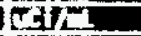 & $\mathrm{N}$ & This:si & $<4.2-2$ & $\sqrt{2}$ & $\vec{n}$ & Ia & 92 & & ma \\
\hline Eisins & 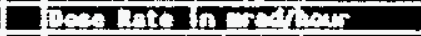 & oxtrets & na & $n$ & $-t+1.0$ & $n$ & & & $\mathrm{~m} \mathbf{0}$ & inent & $\bar{n}$ \\
\hline Eieilouits & Drpearcise a sole & & n/9 & ma & coniete & $a / a$ & [2) & n? & na & na & $\mathrm{na}$ \\
\hline FidGir & GLestbratrotion & CII & 07.30 & $0.49-61$ & $<2.45+3$ & $\mathbf{n a}$ & $n$ & na & $n$ & $2.46 e-63$ & EN.0 \\
\hline 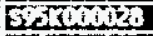 & 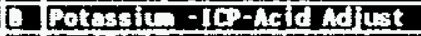 & Lent & 94.00 & $3.92 \mathrm{e-0.23}$ & $<3.03$ & na & na & $\mathbf{n a}$ & 103.1 & 3.00 & na \\
\hline Eistotites & a pu-23. & $\mathbb{a t}$ & $n / a$ & $3.30-13$ & $<2.220-4$ & $\mathbf{n a}$ & I/20 & na & n2a & $2.32-0.9$ & W0.0 \\
\hline 525000322 & 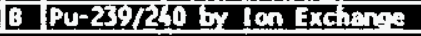 & atr & 09.06 & 3.39e-13 & $<2.22 e-4$ & n/o & na & na & na & $2.22 e-04$ & 100.0 \\
\hline
\end{tabular}

$\stackrel{\leftrightarrow}{\leftrightarrow}$

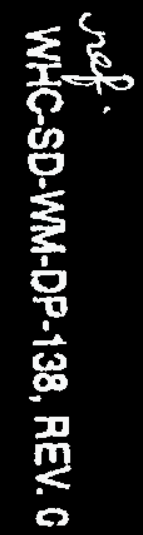


WHC-SD-SNF-DP-003, Rev. 0

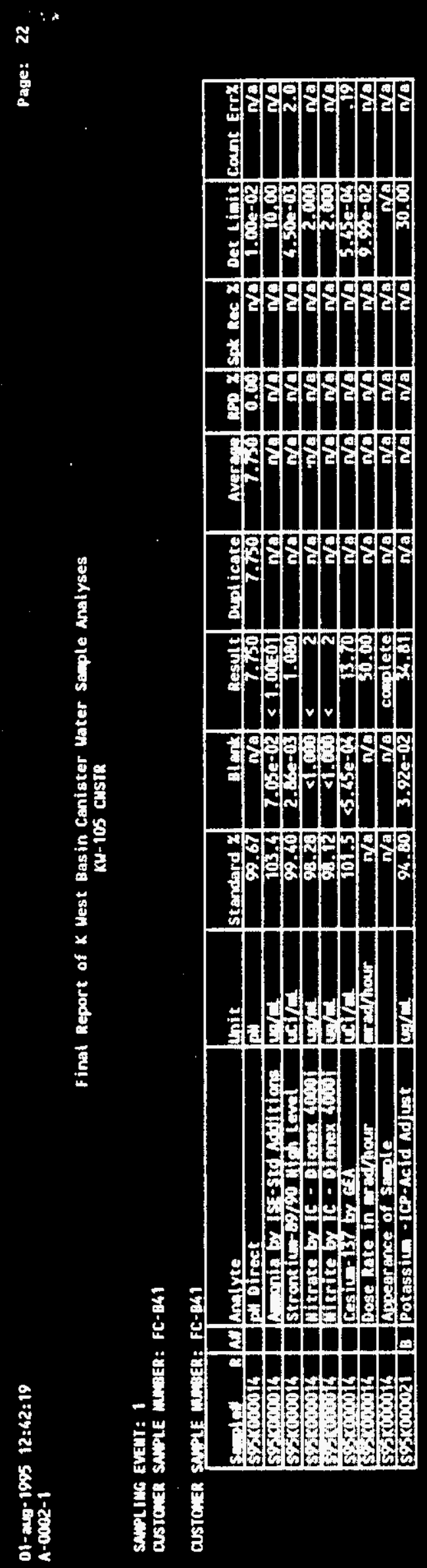

ref.
WHC-SD-WM-OP-138, AEV. O

.35 
Final Report of $K$ Uest Basin Canister Uater Saple Analyses

$$
\mathrm{ku}-105 \text { Custr }
$$

smitus EVEMT: 1

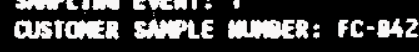

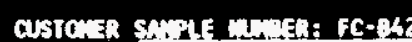

\begin{tabular}{|c|c|c|c|c|c|c|c|c|c|c|c|}
\hline If plet & Mlandre & Init & standard $x$ & Alet: & Berait & Dindicate & Eix & $\sin$ & 28 & & nt Erra \\
\hline Ferotils & SI Drect & 1 & - is.61 & na & $\therefore \quad C_{0} \mathrm{~L}_{3}$ & $n$ & r & $n$ & $n / a$ & & $n / 0$ \\
\hline Findis & Wha 2 Esting & $\operatorname{lng}$ & $\frac{104}{64}$ & & 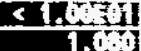 & $m$ & & 29 & $\overline{9}$ & 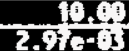 & $\frac{m g}{20}$ \\
\hline 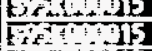 & 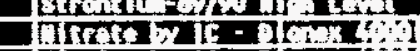 & tris & Qhis & 4.0 & & $\mathrm{ma}$ & 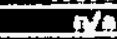 & $\mathrm{ma}$ & 20 & $2 \pi$ & nea \\
\hline $\mathrm{E}+\mathrm{H}$ & 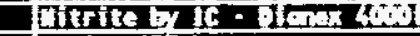 & 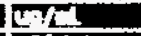 & Q. & $4[1]$ & 5 & mas & 76 & na & $\sqrt{2} \mathrm{a}$ & & $\mathbf{n a}$ \\
\hline WETIID & 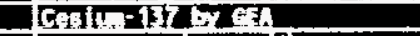 & Gaik: & 101.5 & $6.65 e^{-0}, 4$ & 12.20 & [1...] & FE: & $3 \mathrm{E} \mathrm{E}$ & $n \geq 0$ & & \\
\hline SeNOMT15 & Dose hate increnth & Froldons & ne & $\mathbf{n} / \mathbf{0}$ & 16.0 & $n / 0$ & $n g$ & $\mathbf{n a}$ & nge & 9.9.0202 & na \\
\hline $5+51015$ & noparice of $f$ ins & & $n / 9$ & $\mathbf{n g}$ & coplete & $\mathrm{n} / \mathrm{a}$ & $n 2$ & $n / \Omega$ & $n<2$ & $n / a$ & nes \\
\hline Eistomen & B Cotassin - IcP-Acid ndiest & EFT & 94.60 & $3.922-02$ & $\leq \quad 30.60$ & $n / 9$ & $\mathbf{m a}$ & $\mathrm{Na}$ & $n / 0$ & 30.00 & n/s \\
\hline
\end{tabular}

9

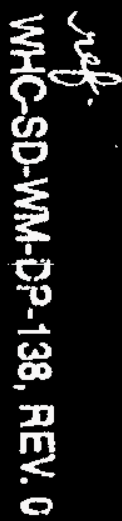


SAMLIWG EVEIT: 1

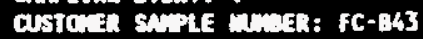

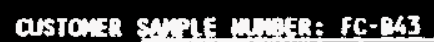

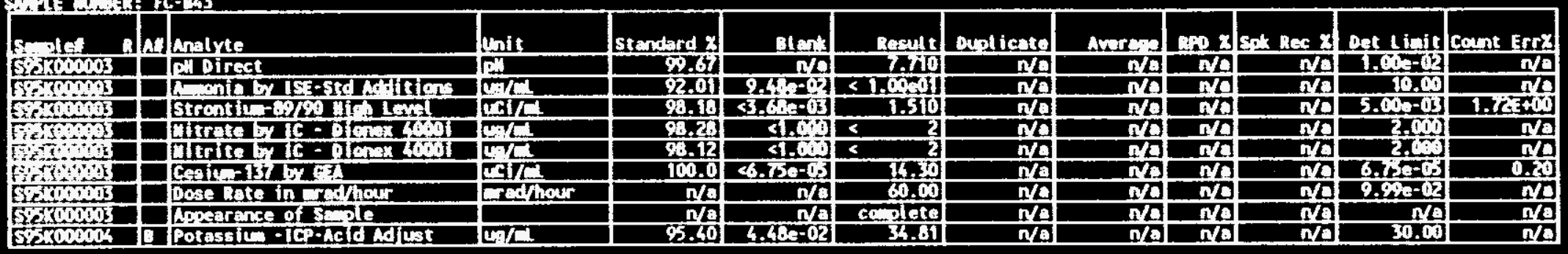

$\omega$ 
Final Report of $K$ West Basin Canister Water Sample Analyses ku-105 custe

\section{SNMLIMG EVEUT: 1}

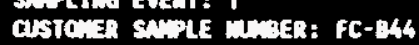

CustonzR SHOLE MESER: FC.R4

\begin{tabular}{|c|c|c|c|c|c|c|c|c|c|c|c|}
\hline sepley & Allanalyse & Lhit & Standard $x$ & Dlent & Besult & Deplicate & Avire: & $\cos x$ & & init & Cont Erry \\
\hline ENThio & Pidrest & 5 & 99.67 & $n$ & 7.300 & $\mathrm{na}$ & Da & no & n:D & $1.00-03$ & $n / \mathbf{a}$ \\
\hline $5 \times-7$ & 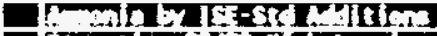 & 11 & Q.01 & a.? & $\leq 1 \mathrm{n}, \mathrm{n}$ & $\pi$ & $n$ & In & $\bar{n}$ & II.0.0 & $n a$ \\
\hline Exisitis & 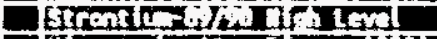 & 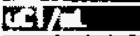 & E..IJ & $\$ . x^{x}$ & $5.2 \leq-01$ & na & ID & $\mathrm{na}$ & ID & $5.100-13$ & 2.95xin \\
\hline Fieiti, & 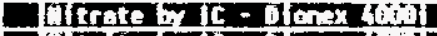 & +2 & 101.2 & 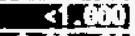 & $<\quad 21$ & $\mathrm{ma}$ & na & $n$ & $\sqrt{2}$ & (7) & $n 20$ \\
\hline Beitosits & 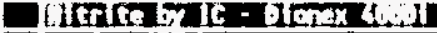 & Ma & $1,0.01$ & C. $.13=011$ & 16.8 & mo & na & $M D$ & $n a$ & & na \\
\hline & EnTsirs 3 & MIE & [0.1] & 4.15:-1 & {$\left[[]^{3}\right]$} & $\sqrt{2}$ & $m a$ & $\mathrm{n}$ & $\bar{m}$ & & 0.10 \\
\hline 5803 & gose nate in rerom & Exthar & $n$ & 20 & 8.00 & aD & $n$ & na & na & Q.sine-02 & $\mathbf{n a}$ \\
\hline Eisioning & tomearence o/ Seple & & $n / 9$ & ng & coplats & a/a & na & $\mathrm{Na}$ & $\mathbf{n g}$ & $\mathbf{n a}$ & no \\
\hline Sistonions & Q Parassion-lcp-hald adout & Gich & 9.60 & $4.48-62$ & $\operatorname{s.17}$ & na & n/a & na & $\mathbf{n a}$ & 30.00 & na \\
\hline
\end{tabular}

is

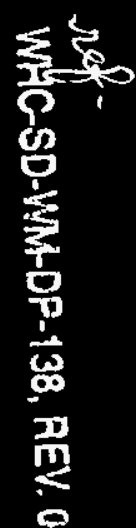


WHC-SD-SWF-DP-003, Rev. 0

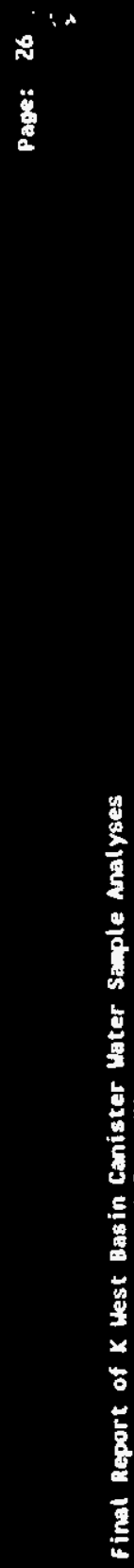

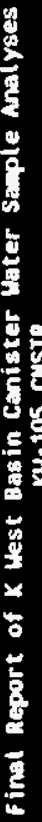

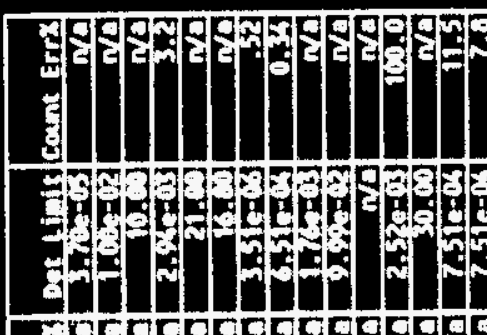

ref.
WHC-SD-WM-DP-138, REV. $\mathrm{C}$

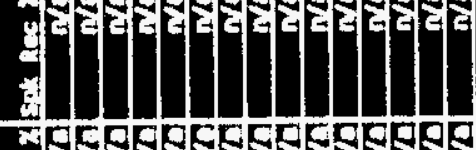

DFF

8

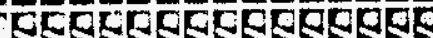

GfEtGGG

3

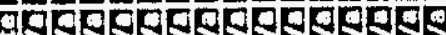

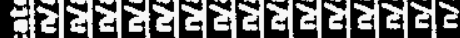

$\frac{0}{3}$

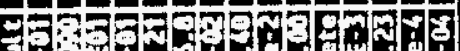

(6)

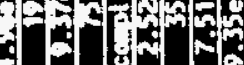

$73 G$ TSFGG

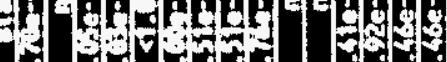

(2) $P$ viv

妾

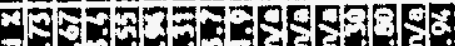

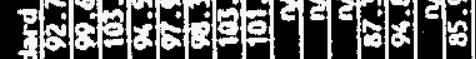

$\Rightarrow$

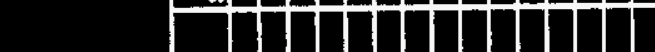

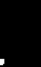

$-1+N d x d$

a.t.

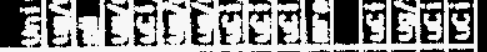

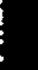

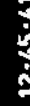

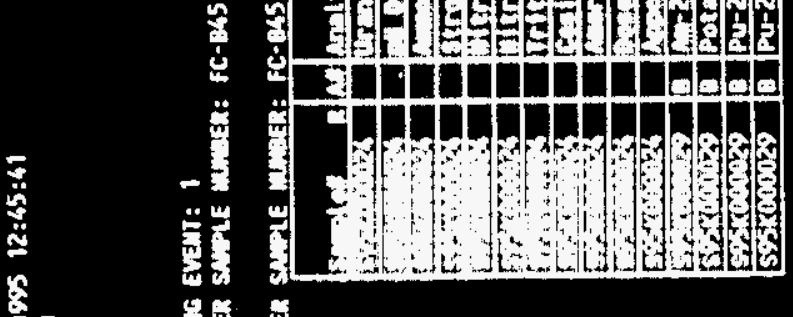




\section{Whe-SD-WM-DP-138, REV. 0}






\begin{tabular}{|c|c|c|c|c|c|c|c|c|c|c|c|}
\hline $8 / 0$ & 20.3001 & g/u & $0 / 0$ & $L 66$ & E/u & $8 / 0$ & e/u & $8 / 0$ & 602 & $220000 \times 565$ & 9va-5d \\
\hline $8 / 0$ & $20-3001$ & $E / \mu$ & 070 & $L 66$ & $8 / 4$ & $8 / 0$ & o/u & EN & $O E L$ & $900000 \times 965$ & $060-34$ \\
\hline $8 / 0$ & $20-3001$ & e/u & eju & L66 & $\mathrm{F} / \mathrm{u}$ & $8 / 0$ & epo & $8 / 0$ & 162 & $800000 \times 565$ & Eta-25 \\
\hline 80 & $20 \cdot 3001$ & equ & ENo & $2 \mathbf{6 6}$ & $8 / 0$ & $8 / 0$ & D/0 & हुण & 082 & $910000 \times 965$ & $268-39$ \\
\hline $8 / 4$ & $20 \cdot 9001$ & g/0 & $\theta 0$ & $\angle 66$ & 000 & $S L L$ & $G L L$ & $S L L$ & SLL & 10000X565 & $168-5$ \\
\hline $8 / u$ & $20 \div 3001$ & E/U & $8 / 0$ & 266 & घ/u & e/u & $8 / 0$ & $8 / 0$ & 832 & $820000 \times 565$ & Ob3.5 \\
\hline E/u & $80-3001$ & $8 / 0$ & काय & $\angle 66$ & $8 / 0$ & $8 / 0$ & $\mathrm{n} / \mathrm{Tu}$ & 870 & 928 & $810000 \times 965$ & 680.54 \\
\hline $8 / 0$ & $20 \cdot 3001$ & Eju & $\nabla / \mathrm{u}$ & 266 & g/u & g/o & $0 / 11$ & $\pi / n$ & 262 & L200001965 & Bet:-2] \\
\hline$\overline{\theta र}$ & 2003007 & mpt- & -70 & $t 66$ & $n \mu$ & Epa & ip/ & Equ & 192 & $210000 \times 945$ & 60021 \\
\hline $8 / 0$ & $20 \cdot 3001$ & $8 / 0$ & Dju & $L 66$ & E/u & $8 / 0$ & $\overline{E \mu}$ & $8 / 0$ & 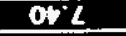 & $110000 \times 965$ & get-34 \\
\hline e/u & $20 \cdot 3001$ & 970 & $8 / 0$ & $L 66$ & tejo & eju & $0 / \mathrm{u}$ & Gh & $6 \% 2$ & $920000 \times 965$ & 028.51 \\
\hline $\mathrm{E} / \mathrm{u}$ & $20-3001$ & $8 / 4$ & D/u & 266 & $8 / \mathrm{G}$ & $8 / 0$ & $0 / 10$ & E/0 & $1 L L$ & 0t0000xs6s & $628-51$ \\
\hline Eju & 20.3001 & E/u & 870 & L66 & $60^{\circ} 0$ & 982 & $98 L$ & 682 & 282 & $600000 \times 965$ & $926 \cdot 53$ \\
\hline EN & $20-3001$ & 8/u & $\nabla \%$ & $\angle 66$ & $0 / 0$ & $8 / 4$ & $8 / \mathrm{u}$ & $8 / 4$ & 882 & $500000 \times 565$ & 968-24 \\
\hline njo & $20-3001$ & $\nabla / \mu$ & 70 & $\angle 66$ & $8 / \mathrm{M}$ & $8 / 0$ & $8 / 10$ & 070 & $89 L$ & SZ0000XS6S & $628 \cdot 2$ \\
\hline $4 / \mu$ & $20-3001$ & $8 / 0$ & $\sigma \mu$ & 2001 & E/0 & $\theta / 0$ & $E / \mathrm{M}$ & $8 \pi$ & 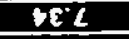 & $860000 \times 156$ & Eखद-J] \\
\hline $8 / 0$ & $20-3001$ & 7/4 & काo & {$[66$} & $8 / 0$ & $\pi / 0$ & ËM & $8 / 0$ & 108 & T0000005SS & 618-34 \\
\hline $8 / 0$ & $20 \cdot 3001$ & E/u & $0 / 0$ & S.00I & $8 / 0$ & $8 / 0$ & g/u & $g / 0$ & 916 & $680000 \times 565$ & 818-34 \\
\hline e/11 & 20.3001 & e/u & $8 / 4$ & sool & $\pi / n$ & $8 / 0$ & $\mathrm{Pm}$ & $\sigma / \mu$ & $\varepsilon ; 8$ & $880000 \times 965$ & 218.5 \\
\hline $8 / 0$ & $20-3001$ & $0 / 4$ & $8 / 0$ & 5001 & काi & g/u & $8 / 0$ & $8 \pi$ & re. & $\angle 80000 \times 56 S$ & $918 \cdot 31$ \\
\hline 7.0 & $20 \cdot 3001$ & E/0 & करण & SOOt & $8 / 4$ & $6 / 0$ & efo & gru & 968 & $960000 \times 565$ & S18-2J \\
\hline $8 / 0$ & 20.5001 & $8 / 0$ & $8 / 0$ & 2001 & 010 & 018 & 018 & 018 & 118 & Lto000XSGS & $018-34$ \\
\hline$\overline{0 / 0}$ & 20.3001 & e/u & $8 / 0$ & 9001 & pjo & $\bar{\theta} / \mathrm{u}$ & $0 / \mathrm{u}$ & 80 & GE' 8 & $580000 \times 565$ & 018.57 \\
\hline E/O & $20-3001$ & 87u & $\pi 0$ & $\mathrm{~g}^{\circ} 00 \mathrm{l}$ & $8 / 4$ & $8 / 0$ & घ/4 & E/ & 692 & $080000 \times 965$ & $\angle 00-23$ \\
\hline g/0 & $20 \cdot 3001$ & 80 & Qp & 5001 & $8 / \bar{u}$ & $8 / 0$ & en & en & IBE & $860000 \times 965$ & $200 \cdot 3 t$ \\
\hline 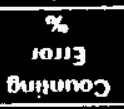 & 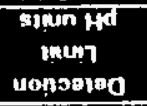 & 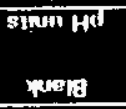 & $\begin{array}{c}\text { \% } \\
\text { Aronosoy } \\
\text { oxids }\end{array}$ & $\begin{array}{c}\% \\
\text { Alonosey } \\
\text { prepunis }\end{array}$ & $\begin{array}{c}\text { AStic } \\
\text { uossiostd } \\
\text { gphues }\end{array}$ & $\begin{array}{l}\text { somon ifd } \\
\text { weaw } \\
\text { ophoes }\end{array}$ & 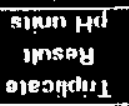 & 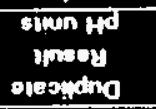 & $\begin{array}{l}\text { stin Hd } \\
\text { uneoy } \\
\text { paysu }\end{array}$ & $\begin{array}{l}\text { sequank } \\
\text { Asoseroctis? }\end{array}$ & 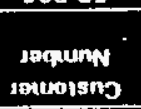 \\
\hline & 70 & $80 \quad$ AIIT & 5 TVIIAT & & & $\mathbf{s}$ & SI8 SISA & jy 37 wivs & & NOIIVJHIN & 1 J7devs \\
\hline
\end{tabular}




\begin{tabular}{|c|c|c|c|c|c|c|c|c|c|c|c|}
\hline E/N & $10+3007$ & $20.350 L$ & Eम & $b^{+} \mathrm{EOL}$ & च/1 & $8 / 0$ & E/u & E/u & $10+3001>$ & 120000XS6S & Sta-5z \\
\hline $0 \%$ & $10+3001$ & $20-3865$ & बार & 026 & $8 / 4$ & E/u & D/u & $8 / 0$ & $10+3001>$ & $900000 \times 565$ & $1+0.34$ \\
\hline $8 \mathrm{g0}$ & $10+3001$ & $20-3866$ & $6 / \mathrm{tu}$ & 026 & $0 / 0$ & 70 & efu & $8 / 0$ & $10+300.1>$ & $800000 \times 565$ & $868 \cdot 3$ \\
\hline 80 & $10+3001$ & $20.390 \mathrm{~L}$ & eft & 6801 & $0 / 0$ & Fu & E/u & $8 / 4$ & $10+300 t>$ & $910000 \times 565$ & $260-2 t$ \\
\hline $8 / 4$ & $10+300 \mathrm{~L}$ & $20.150 \mathrm{~L}$ & घ70 & 6801 & Efu & $0 / 0$ & हjo & E/u & $10+3001>$ & $10000 \times 565$ & $160-34$ \\
\hline$\theta \mu$ & $10+3001$ & $20 \cdot 390.2$ & E/0 & $80 t$ & $\bar{\sigma} / \mathrm{u}$ & $8 \mathrm{u}$ & $8 / 0$ & $\theta \mu$ & $10+3001>$ & $820000 \times 565$ & $0+8-24$ \\
\hline E. & $10+3001$ & $20-3606$ & E/u & 0.26 & E/u & D/u & $\pi / 0$ & E/u & $10+3021$ & c10000056s & $6 E 0 \cdot 32$ \\
\hline $8 / \mu$ & $10+3001$ & $20-390 L$ & $8 / 4$ & 6001 & $8 / 0$ & $8 / 0$ & E/0 & Eju & $10+300 t>$ & $220000 \times 565$ & $8 c 8 \cdot 5 d$ \\
\hline Ep & $10+3001$ & $20-3846$ & Eja & 075 & Ep & $8 / 0$ & $8 / 4$ & g/o & $10+300 t>$ & $210000 \times 565$ & LE8-5t \\
\hline e/u & $10+3001$ & $20.350 L$ & 296 & PEOt & E/u & 80 & $10+300.1>$ & $10+3001>$ & $10+3001>$ & 110000xass & 980.3 \\
\hline Eju & $10+3001$ & 20.3502 & $\mathrm{Bg} \mathrm{C}$ & 6201 & $8 / 0$ & 80 & $8 / \mathrm{M}$ & $8 \mathrm{pu}$ & $10+300 t>$ & $920000 \times 965$ & $028 \cdot 5$ \\
\hline e/u & $10+300 i$ & 20.3866 & 996 & 026 & E/U & $8 / 0$ & $10+300.1>$ & $10+3001>$ & $10+3001>$ & $010000 \% 968$ & 620.3A \\
\hline $8 / u$ & $10+3001$ & $20-3866$ & jou & 026 & $8 / 0$ & epu & $0 / 0$ & हnu & $10+3001>$ & $600000 \times 155$ & $928 \cdot 24$ \\
\hline Eu & $10+3001$ & $20-38+6$ & हर & 026 & $8 / \mathrm{M}$ & $8 / 0$ & ह $/ 0$ & 8/u & $10+3001>$ & 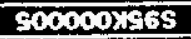 & 520:54 \\
\hline $8 / \mathrm{O}$ & $10+300 !$ & $20-3902$ & Oju & 5801 & घju & $8 / 4$ & E/u & $8 / 0$ & $10+3001>$ & S20000X56s & $60-34$ \\
\hline $\bar{E} \boldsymbol{e} \tilde{T}$ & $00+3009$ & $10-30027$ & 0.001 & $0 \mathrm{sol}$ & $8 \mathrm{Pu}$ & $8 \pi$ & $\infty+300,5>$ & $00+3009>$ & $00+3005>$ & Droo00065s & $860-24$ \\
\hline $8 / 0$ & $10+300 t$ & 203866 & E/1i & 026 & घh0 & Diu & $8 / 0$ & $\theta / \mathrm{u}$ & $10+300 t>$ & $100000 \times 565$ & $618 \cdot 5 \mathrm{y}$ \\
\hline $8 / 0$ & $00+300 \mathrm{G}$ & $10-30027$ & $8 / 0$ & 0.501 & $8 \%$ & 80 & E/0 & $8 / 0$ & $00+3009>$ & 650000NG65 & 810.24 \\
\hline D/u & $00+3009$ & $10-30072$ & J/u & $0 \mathrm{SOI}$ & घha & g/u & $0 / 0$ & apu & $00+5005>$ & $880000 \times 565$ & $\angle 18 \cdot 94$ \\
\hline epu & $00+3009$ & 1030028 & $\nabla \mu$ & $0 \mathrm{GOI}$ & $E \mu$ & $8 / 0$ & $\theta / \mu$ & $\overline{\theta \mu}$ & $00+5005>$ & $\angle 80000 \times 965$ & 918.53 \\
\hline$\sigma \pi$ & $00+3005$ & $10 \cdot 1007>$ & $\theta / \mu$ & OSOL & $8 / 0$ & $8 / 0$ & $0 / 0$ & $\mathrm{Bfu}$ & $00+3009>$ & $080000 \times 565$ & 918.51 \\
\hline E/u & $00+3009$ & $10-3007>$ & $8 / 0$ & osol & g/0 & diu & $8 / 0$ & D/u & $00+300 s>$ & Lroo00XSGS & $518-39$ \\
\hline हरा & $00+3005$ & $10-30027$ & $\theta / \mathrm{u}$ & 0901 & Dju & efu & Eju & 8/0 & $00+3005>$ & $980000 \times 565$ & $018 \cdot 3$ \\
\hline हरा & $00+300 \mathrm{~s}$ & $10 \cdot 30027$ & 80 & 0501 & $\sigma \mu$ & E/u & B/u & $8 / 0$ & $00+3009>$ & $780000 \times 565$ & $200 \cdot 3$ \\
\hline ह10 & $\infty 0+3009$ & $10-30025$ & 80 & 0.901 & $\mathrm{NN}$ & $8 / 0$ & E/v & $0 / 0$ & $00+5009>$ & $860000 \times 565$ & $200 \cdot 5$ \\
\hline 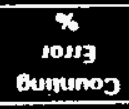 & 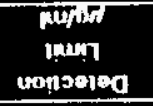 & pawa & $\begin{array}{c}\bar{x} \\
\text { Avenosoy } \\
\text { oxnds }\end{array}$ & $\begin{array}{c}\% \\
\text { Anenosoy } \\
\text { prepueis }\end{array}$ & 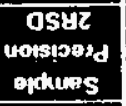 & 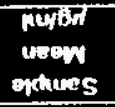 & 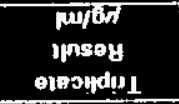 & 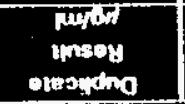 & 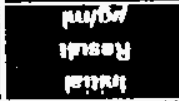 & $\begin{array}{l}\text { secpums } \\
\text { A soiveroqe? }\end{array}$ & $\begin{array}{l}\text { sequinin } \\
\text { seusolsing }\end{array}$ \\
\hline & 70 & tNo5 AIIT & 5 TYIIX] & & & & InSJU SISAT & WV Jlonvs & & MOIIVJIIIN & 1 37denvs \\
\hline
\end{tabular}

\begin{tabular}{|c|c|c|c|c|c|c|c|c|c|c|c|}
\hline Ejo & $10+3007$ & $20: 3902$ & हुu & 600 & $\nabla / u$ & $8 / 0$ & E/u & 8/u & $10+3001>$ & $120000 \times 565$ & S6g-5d \\
\hline ס0 & $10+3001$ & $20-3806$ & ब/v & 026 & $8 / 4$ & $8 / \mu$ & $\nabla / \mathrm{u}$ & $8 / 0$ & $10+3001>$ & $900000 \times 565$ & 00.24 \\
\hline $8 \mathrm{fo}$ & $10+3001$ & $20-3866$ & g/u & 026 & $0 / 0$ & 70 & eju & $8 / 0$ & $10+3001>$ & $800000 \times 565$ & $8 \times 9 \cdot 3$ \\
\hline 80 & $10+300 t$ & 203902 & Efu & 6801 & $0 / 0$ & हu & E/u & $0 / 4$ & $10+3001>$ & $910000 \times 565$ & $2+0-3 t$ \\
\hline $8 / 4$ & $10+300 t$ & $20.150 \mathrm{~L}$ & घ/0 & 6801 & E/u & $0 / 4$ & हjo & E/0 & $10+300 \mid>$ & $10000 \times 565$ & $160-34$ \\
\hline$\theta \mu$ & $10+3001$ & $20 \cdot 390.2$ & $8 / 0$ & 6801 & ofu & $8 \mathrm{gi}$ & $8 / 0$ & 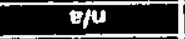 & $10+3001>$ & $820000 \times 565$ & $0,8-24$ \\
\hline E. & $10+3001$ & $20-3366$ & $\theta / u$ & 0.26 & E/u & D/u & $\pi / 0$ & E/0 & $10+3021$ & $810000 \times 565$ & $6 \times 0.34$ \\
\hline 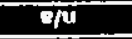 & $10+300 t$ & $20-390^{\circ} \mathrm{L}$ & $8 / 4$ & 6001 & $8 / 0$ & $8 / 0$ & E/0 & Eju & $10+300 t>$ & $220000 \times 565$ & $8 c 8 \cdot 5 d$ \\
\hline EA & $10+300 t$ & $20-3846$ & E/u & 026 & $\mathrm{EF}$ & $8 / 0$ & $8 / \mathrm{u}$ & oju & $10+3001>$ & $210000 \times 965$ & LE8-5t \\
\hline e/u & $10+3001$ & 20.3902 & 296 & 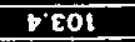 & E/u & 80 & $10+3001>$ & $10+3001>$ & $10+3001>$ & 110000xass & 980.3 \\
\hline Eju & $10+3001$ & 20.3502 & $8 / 0$ & 6801 & $0 / 0$ & eju & $8 / \mathrm{u}$ & $8 \mathrm{pu}$ & $10+3001>$ & $920000 \times 965$ & 0os:2 \\
\hline E/0 & $10+3000$ & 20.3866 & 996 & 026 & E/U & $8 / 0$ & $10+3001>$ & $10+3001\rangle$ & $10+3001>$ & $010000 \times 965$ & $628 \cdot 5$ \\
\hline $8 / u$ & $10+3001$ & $20-3866$ & g/u & 026 & $8 / 0$ & epu & $8 / 0$ & Epu & $10+3001\rangle$ & $600000 \times 958$ & $928 \cdot 24$ \\
\hline Eu & $10+3001$ & $20-3806$ & हर & 026 & $8 / \mathrm{M}$ & $8 / 0$ & ह $/ 0$ & 8/u & $10+300 t>$ & 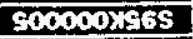 & SE:-2: \\
\hline $8 / 0$ & $10+300 !$ & $20-3902$ & g/u & 5801 & घju & $8 / 4$ & B/u & $8 / 0$ & $10+3001>$ & S20000xiss & $260-31$ \\
\hline E/tút & $00+300 \mathrm{~s}$ & $10-30073$ & 0.001 & $0 \mathrm{sol}$ & $8 \mathrm{Fu}$ & $8 \pi$ & $00+300,5>$ & $00+3009>$ & $00+300 \mathrm{~s}>$ & 6ro000xS6S & हर:-2y \\
\hline 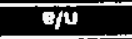 & $10+300 t$ & 203866 & बहाi & 026 & घju & Eju & 80 & $8 / \mu$ & $10+300 t>$ & $100000 \times 565$ & $618 \cdot 5$ \\
\hline $8 / 0$ & $00+300 \mathrm{~s}$ & $10-30027$ & $8 / 0$ & 0.501 & $8 \%$ & 80 & $8 / 0$ & घ/0 & $00+3009>$ & $680000 \times 565$ & $818 \cdot 24$ \\
\hline D/4 & $00+3009$ & $10-30072$ & $0 / 0$ & $0 \mathrm{SOL}$ & $8 \mathrm{ju}$ & g/u & $8 / 0$ & apu & $00+300 s>$ & $880000 \times 565$ & $\angle 18 \cdot 54$ \\
\hline EN & $00+300 s$ & $10-30028$ & $\nabla \mu$ & $0 \mathrm{SOI}$ & $E \mu$ & 80 & $8 / \mu$ & $\overline{\theta u}$ & $00+300 \mathrm{~S}>$ & L80000XSGS & 918.54 \\
\hline DN & $00+3005$ & $10 \cdot 3007>$ & $\theta / \mu$ & OSOL & $0 / 0$ & E/0 & $0 / 0$ & काu & $00+3009>$ & $080000 \times 565$ & 910.5 \\
\hline E/u & $00+3009$ & $10-30027$ & $8 / 0$ & 0.901 & Ejo & g/u & $8 / 0$ & D/u & $00+3009>$ & Lro000XSGS & 618-34 \\
\hline नju & $00+3005$ & $10-30072$ & ब/u & 0901 & $\overline{D T u}$ & E/u & g/u & 8/0 & $00+3009>$ & $980000 \times 565$ & $018 \cdot 29$ \\
\hline हरा & $00+300 \mathrm{~s}$ & $10 \cdot 30027$ & 80 & 0901 & बמ & Eju & $8 / 0$ & 8/u & $00+300 \mathrm{~S}$ & DE0000X56s & $200 \cdot 31$ \\
\hline हु० & $\infty+3009$ & $10-20025$ & $8 \mathrm{pu}$ & 0 SOI & $\mathrm{N} / \mathrm{N}$ & $8 \pi$ & $8 / 0$ & $0 / 0$ & $60+5009>$ & $860000 \times 565$ & 209.54 \\
\hline Ouyunos & $\frac{\text { conseied }}{70}$ & 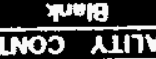 & $\frac{0 \times 45}{7 V \text { iI }}$ & & & oxtures & 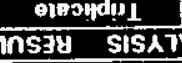 & oresting & Find & NOIVJIEHN & J7dowrs \\
\hline
\end{tabular}

\begin{tabular}{|c|c|c|}
\hline $8 / 0$ & Eju & $8 / 0$ \\
\hline $8 / \bar{d}$ & $\mathrm{D} / \mathrm{u}$ & $8 / 0$ \\
\hline$\sigma \sigma^{u}$ & $8 / 11$ & $8 / 0$ \\
\hline हाu & $8 / 4$ & $0 / 4$ \\
\hline $8 / \mathrm{u}$ & g/o & $\mathrm{E} / \mathrm{U}$ \\
\hline Ecu & $\bar{\delta} \mathrm{u}$ & 80 \\
\hline$\overline{0} / \mathrm{u}$ & $\nabla / 0$ & $8 \mathrm{du}$ \\
\hline $8 / 0$ & $8 / 0$ & $\mathrm{e} / \mathrm{u}$ \\
\hline
\end{tabular}

$10+3001>$

\begin{tabular}{l|l|l}
$3001>$ & $120000 \times 965$ & $960-57$ \\
\hline
\end{tabular} \begin{tabular}{ll}
$001>00000 \times 565$ & $1+0.5$ \\
\hline
\end{tabular}

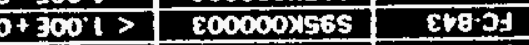
\begin{tabular}{l|l|l|l}
$10+3001>$ & $910000 \times 565$ & $260-2 t$
\end{tabular} $10+3001>\quad+10000 \times 565 \mid 160-3$ $10+3001>$ ez0000xs5s 0ra-2y

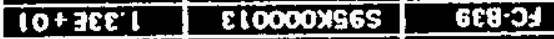
fu $\quad 10+300 t>\quad$ L20000)

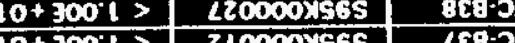
$0 \cdot 7100 \cdot 189-77$

10010 


\section{sef. \\ WHC-SD-WM-DP-138, REV. 0}

WHC-SD-SNF-DP-003, Rev. 0

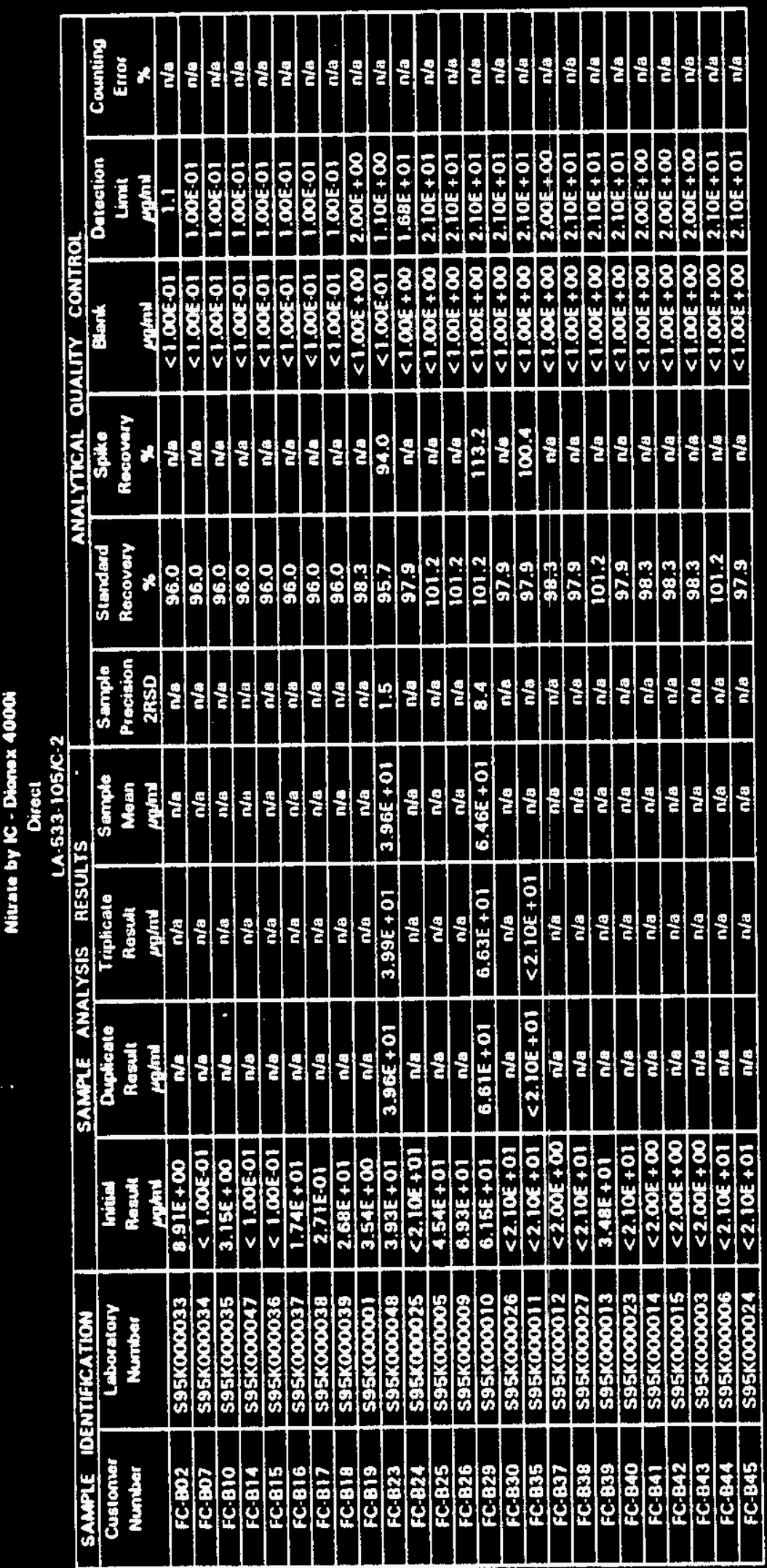

.73 


\begin{tabular}{|c|c|c|c|c|c|c|c|c|c|c|c|}
\hline 800 & $10+3891$ & $10-30087$ & Epo & 886 & $e / 0$ & $8 \pi$ & $8 / \mathrm{u}$ & 70 & $10+3091>$ & $120000 \times 15 S$ & 560.53 \\
\hline $8 / 0$ & $\frac{10+389 t}{00+3007}$ & \begin{tabular}{|l}
$10 \cdot 300 \cdot 8>$ \\
$00+30011>$
\end{tabular} & $8 \mathrm{u}$ & 0001 & 80 & 80 & $8 / \mathrm{J}$ & $8 / 0$ & $10+3691>$ & $900000 \times 565$ & P6a.23 \\
\hline$\frac{80}{8 / 0}$ & $\frac{00+3007}{00+300 z}$ & $\frac{\infty 0+30011}{\infty 0+30011}$ & $\frac{8 \pi 0}{8 / 4}$ & 186 & $8 / 0$ & $8 / 0$ & $8 / 0$ & $8 \pi$ & $00+3002>$ & $800000 \times 965$ & Eva.j \\
\hline $8 / 0$ & $\frac{00+300 z}{00+300 z}$ & $\frac{\infty+300^{\prime} \mid>}{\infty+300 ! 1}$ & $\frac{8 \pi u}{2 j u}$ & 186 & $8 / \mathrm{u}$ & Eju & $8 / \mathrm{u}$ & $8 / 0$ & $00+3002>$ & S10000X565 & 2609 \\
\hline $8 / 0$ & $\frac{00+3007}{10+3891}$ & \begin{tabular}{|l|}
$00+3001>$ \\
$10 \cdot 300,8>$
\end{tabular} & $\frac{\sigma j u}{\sigma / u}$ & 186 & $8 / 0$ & $8 / 4$ & $8 / \mathrm{u}$ & Eptu & $00+300 z>$ & DLO000XSGS & 168.24 \\
\hline $8 / \mathrm{u}$ & $\frac{10+389 \cdot 1}{10+389 \cdot 1}$ & $\frac{10 \cdot 3008>}{10 \cdot 30087}$ & $\frac{8 \pi u}{8 / 4}$ & $\varepsilon^{\prime 86}$ & $8 / 0$ & g/u & $8 / \mathrm{u}$ & $8 / 0$ & $10+3891>$ & $820000 \times 565$ & 04.53 \\
\hline $8 / \mu$ & $\frac{10+3899}{10+3891}$ & $\frac{10 \cdot 30087}{10 \cdot 30088}$ & 8 & 0.001 & 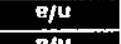 & $8 \mathrm{M}$ & Ef" & $8 \mu$ & $10+3891>$ & $810000 \times 965$ & $608-34$ \\
\hline$g \pi$ & $00+7002$ & $00+300 \mid>$ & F/u & $\frac{8^{8} 86}{1.86}$ & E/u & epu & $8 / 0$ & $8 / 0$ & $10+3891>$ & L60000XSGS & 8E8.5 \\
\hline $8 / \bar{u}$ & $10+3897$ & $10-3008>$ & $8<6$ & $\frac{186}{8.86}$ & $8 / 4$ & $8 / 0$ & e/u & $\frac{70}{20}$ & $00+3002>$ & $210000 \times 565$ & $\frac{100-32}{\cos 25}$ \\
\hline $8 / 4$ & $10+3897$ & $10-3008>$ & $5 / 0$ & $\frac{886}{8886}$ & $\frac{8 / u}{8 j u}$ & $\frac{8 j u}{8 j u}$ & $\frac{10+389 \cdot 1>}{8 / 0}$ & $\frac{10+38991>}{8 / 4}$ & $\left.\frac{10+369 t>}{10+389 t}\right\rangle$ & $\frac{110000 \times 585}{960000 \times 965}$ & $\frac{0,8-29}{5 \times 8.35}$ \\
\hline 870 & $10+3891$ & $103008>$ & SOOI & 0.001 & $8 / 0$ & $\sigma \pi$ & $10+389 \mid>$ & $10+389 \cdot 1>$ & $10+7691>$ & $\frac{9200000 \times 565}{010000 \times 565}$ & $\frac{0.89 \cdot 3}{620 \cdot 54}$ \\
\hline $8 / 5$ & $10+389 t$ & $103008>$ & $8 / 0$ & 0.001 & $8 / 0$ & o/u & $\mathrm{g} / \mathrm{u}$ & $8 / \mathrm{u}$ & $10+369 t>$ & $600000 \times 565$ & $988-3 d$ \\
\hline $8 \pi$ & $10+3 B 91$ & 1030083 & 80 & 0.001 & $8 / 0$ & $8 \pi$ & $8 / 4$ & $8 / v$ & $10+369 t>$ & $500000 \times 565$ & S6:-34 \\
\hline $8 \pi$ & $10+3391$ & 10.30087 & 90 & 886 & $\overline{\mathrm{bju}}$ & e/u & Epu & $8 \bar{u}$ & $10+389 t>$ & $920000 \times 565$ & $628-23$ \\
\hline Dpi & $\infty+3011$ & $10 \cdot 3001>$ & $L \mathbf{6 8}$ & 1.96 & $\overline{C E}$ & $\bar{\infty}+$ IEE'E & $00+38 \tau^{\prime} \varepsilon$ & $00+300$ & $00+30 \varepsilon^{\prime} \varepsilon$ & $800000 \times 565$ & $828-5$ \\
\hline $\mathrm{p \mu}$ & $00+3002$ & $00+3001>$ & ot & 786 & 7ju & $\theta / \mathrm{N}$ & घu & gu & $00+3092$ & $100000 \times 558$ & $618 \cdot 3 \mathrm{H}$ \\
\hline $8 \pi$ & $10 \cdot 3001$ & $10.3001>$ & Dju & 956 & $8 / 0$ & v/u & $8 \pi$ & $8 / 0$ & 10.3212 & $680000 \times 565$ & $818-34$ \\
\hline$\frac{\sigma w}{\partial j}$ & $10=3001$ & 10.30011 & $0 \pi$ & 956 & 80 & $8 / u$ & $8 / u$ & $5 / 0$ & $10.3001>$ & 8200002065 & $\angle I 0-3 t$ \\
\hline 50 & $\frac{10-300^{1} 1}{10-300^{\prime} t}$ & $10=3001>$ & \% & 996 & $8 / 4$ & $E \pi$ & $8 / \mu$ & $\pi$ & $00+360 t$ & LCocoons $6 \mathrm{~S}$ & 970.34 \\
\hline $8 / 0$ & $\frac{10-300 t}{10-300 t}$ & $10-30012$ & 70 & 996 & $8 / 4$ & $8 \mu$ & $\theta \mu$ & 80 & $10.3001>$ & $980000 \times 965$ & $518 \cdot 23$ \\
\hline To & $\frac{10 \cdot 3001}{10 \cdot 3001}$ & $10 \cdot 300 \mid>$ & $8 \pi \mathrm{u}$ & 996 & E/0 & $8 \mathrm{gu}$ & E/11 & $8 / 0$ & $10-3001>$ & L00000NS6S & D18-53 \\
\hline Dल & $\begin{array}{l}103001 \\
103001\end{array}$ & $\frac{10 \cdot 30011}{10 \cdot 300 ! 1}$ & $0 \pi 0$ & 9.56 & 8jo & 80 & $8 / u$ & E/u & $00+3012$ & S80000056S & $018-5$ \\
\hline 8,0 & $00+301$ & $\frac{10 \cdot 30011>}{10-300 \cdot 1>}$ & $8 / 4$ & 956 & Eju & $87 \mathrm{u}$ & $8 / \mu$ & $8 \pi$ & $103001>$ & 180000XS6S & 200.54 \\
\hline & & 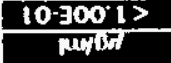 & pu & 996 & $8 / 4$ & 80 & 8/u & $0 / 0$ & $\infty+38 z^{2}$ & EE0000x56s & 200.54 \\
\hline $\begin{array}{l}\text { sous } \\
\text { Beypunos } \\
\end{array}$ & $\begin{array}{c}\text { mect } \\
\text { uppiseseg } \\
\end{array}$ & 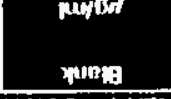 & 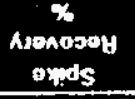 & $\begin{array}{l}\% \\
\text { Aserosey } \\
\text { perpuris }\end{array}$ & 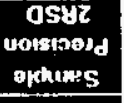 & 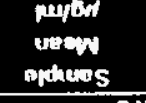 & 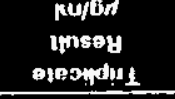 & 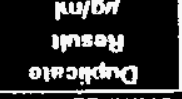 & 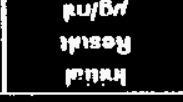 & $\begin{array}{l}\text { soquink } \\
\text { Asplesoqp }\end{array}$ & $\begin{array}{l}\text { soquann } \\
\text { souvisno }\end{array}$ \\
\hline & & InOS AIIT & 0 Triोi & & & & InSEY SISAT & WV jowys & & Nolly & a 3hinvs \\
\hline
\end{tabular}




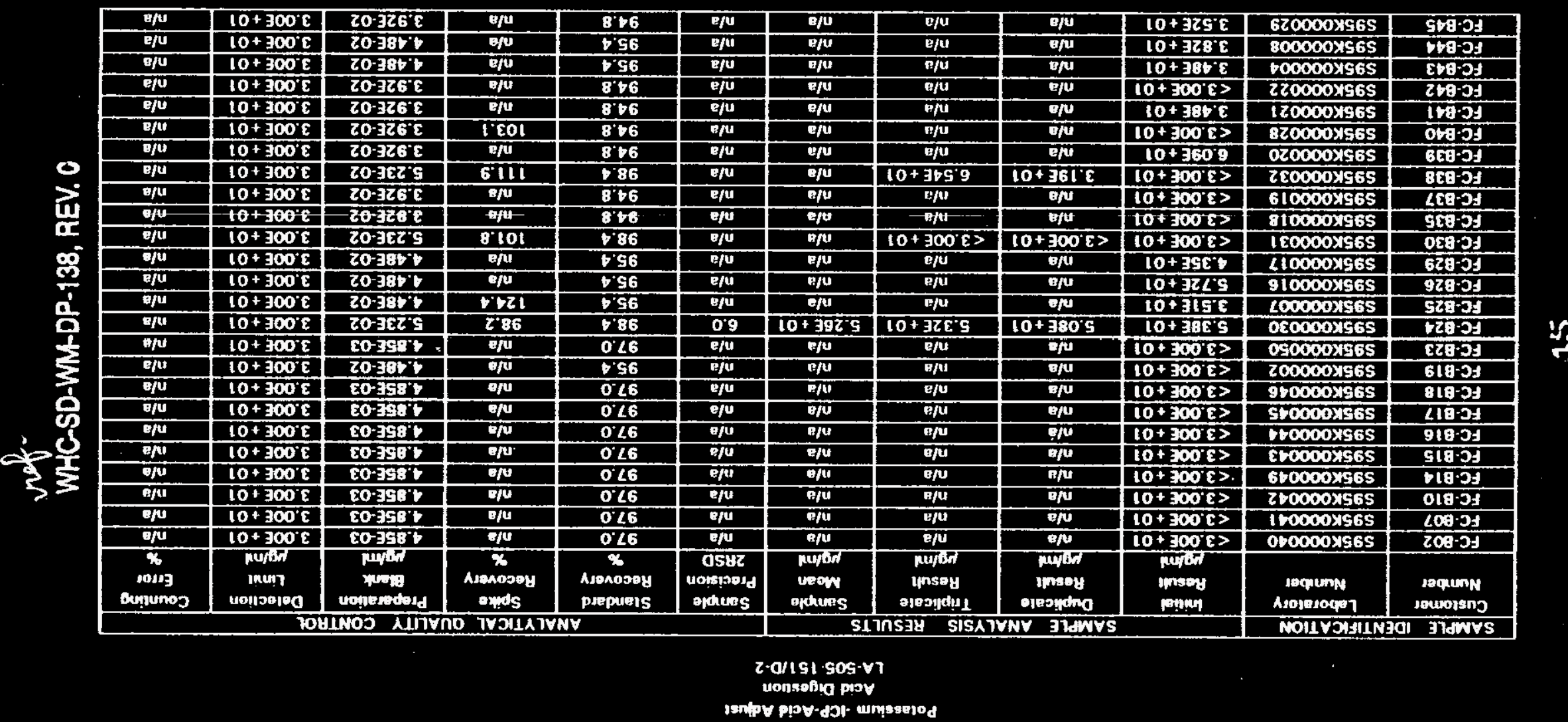


Uranium by Phosphorescence

Direct

LA-925-009/A-O

\begin{tabular}{|c|c|c|c|c|c|c|c|c|c|c|c|}
\hline \multicolumn{2}{|c|}{ SAMPLE IDENTIFICATION } & \multicolumn{4}{|c|}{ SAMHL ANALYSIS AESULTS } & \multicolumn{6}{|c|}{ ANALYTKCAL OUALTY COMTROL } \\
\hline $\begin{array}{l}\text { Cusionver } \\
\text { Number }\end{array}$ & $\begin{array}{c}\text { Leboratory } \\
\text { Number }\end{array}$ & $\begin{array}{l}\text { Initial } \\
\text { Rosult } \\
\text { Lathl }\end{array}$ & $\begin{array}{c}\text { Duplicato } \\
\text { Result } \\
\text { pontul }\end{array}$ & $\begin{array}{c}\text { Triplicate } \\
\text { Result } \\
\text { po/mul }\end{array}$ & $\begin{array}{c}\text { Semplo } \\
\text { Moan } \\
\text { motum }\end{array}$ & $\begin{array}{c}\text { Sampla } \\
\text { Precision } \\
\text { 2ASD } \\
\end{array}$ & $\begin{array}{c}\text { Standard } \\
\text { Recovery } \\
\%\end{array}$ & $\begin{array}{c}\text { Spike } \\
\text { Rocovery } \\
x\end{array}$ & $\begin{array}{l}\text { Blank } \\
\text { paton' }\end{array}$ & $\begin{array}{c}\text { Defection } \\
\text { Limil } \\
\text { gumul }\end{array}$ & $\begin{array}{c}\text { Counting } \\
\text { Error } \\
\%\end{array}$ \\
\hline FC- 8007 & $595 \times 000034$ & $<3.705-04$ & no & $n / a$ & $\mathrm{n} / \mathrm{a}$ & na & 102.4 & $\mathrm{n} / \mathrm{a}$ & $<3.70 .04$ & $3.70 E-04$ & $n / 5$ \\
\hline FC. B10 & 5930000035 & $9.431-01$ & n/a & $\mathrm{n} / \mathrm{a}$ & $n / a$ & $n / \mathbf{a}$ & 102.4 & n/a & $<3.70=04$ & $3.70 E \cdot 04$ & n/a \\
\hline FC. 315 & S95K000036 & $1.39 E+\infty 0$ & n/a & $n / 3$ & $n / a$ & $n / a$ & 102.4 & no & $<3.70 \mathrm{E} .04$ & $3.70 E \cdot 04$ & $\mathrm{n} / \mathrm{a}$ \\
\hline FC-824 & $595 \times 000025$ & $1.60 t+\infty$ & $1.43 E+\infty 0$ & $1.49 E+00$ & $1.51 E+\infty$ & 11.4 & 103.1 & 161.0 & $7.92 E-04$ & $3.70 E-05$ & $n / \mathrm{s}$ \\
\hline FC-B30 & $595 \times 000026$ & $1.58 E+\infty$ & $1.58 E+\infty 0$ & $1.56 E+\infty 0$ & $1.57 E+\infty$ & 1.5 & 92.7 & 133.0 & $<3.700-05$ & 3.70E-05 & $n / a$ \\
\hline FC-BBB & S835000027 & $3.38-01$ & $3.32 E-01$ & 3.38E-01 & $3.36 E-01$ & 2.1 & 92.7 & 132.0 & $<3.701-05$ & $3.70 E .05$ & $n / g$ \\
\hline FC-BAO & S95K000023 & $4.40 E+00$ & $n / 0$ & $n / a$ & $n / a$ & $n / a$ & 103.1 & n/a & $7.92 E-04$ & $3.70 \mathrm{E}-05$ & $n / a$ \\
\hline FC-BAS & S8EK000024 & 7.92E-01 & n/o & $n / b$ & $n / a$ & $n / a$ & 92.7 & alo & $<3.70 E-05$ & 3.70E-05 & $\mathrm{n} / \mathrm{a}$ \\
\hline
\end{tabular}

$\frac{5}{3}$ 
Strontium-89reo thot Level

Direct

(A-220.1010.1

\begin{tabular}{|c|c|c|c|c|c|c|c|c|c|c|c|}
\hline \multicolumn{2}{|c|}{ SAMPIE IOENTIFICATION } & \multicolumn{4}{|c|}{ SAMPLE ANALYSIS RESULTS } & \multicolumn{6}{|c|}{ ANALYTICAL QUALITY CONIROL } \\
\hline $\begin{array}{l}\text { Customer } \\
\text { Nunber }\end{array}$ & $\begin{array}{c}\text { Latroratory } \\
\text { Nunber }\end{array}$ & $\begin{array}{l}\text { Initiel } \\
\text { Rasule } \\
\text { ICiknt }\end{array}$ & $\begin{array}{c}\text { Ouplicate } \\
\text { Rosult } \\
\text { PCiknt }\end{array}$ & $\begin{array}{c}\text { Triplicale } \\
\text { Resulh } \\
\text { rCimn }\end{array}$ & $\begin{array}{l}\text { Sample } \\
\text { Moan } \\
\text { Cintul }\end{array}$ & $\begin{array}{c}\text { Sampla } \\
\text { Precision } \\
\text { 2RSD }\end{array}$ & $\begin{array}{c}\text { Slandard } \\
\text { Racovery } \\
\%\end{array}$ & $\begin{array}{c}\text { Spine } \\
\text { Rocovery } \\
\mathbf{x}\end{array}$ & $\begin{array}{l}\text { Blank } \\
\text { rCi/nnl }\end{array}$ & $\begin{array}{l}\text { Detection } \\
\text { Limit } \\
\text { chind }\end{array}$ & $\begin{array}{c}\text { Counting } \\
\text { Error } \\
\%\end{array}$ \\
\hline FC. $\mathbf{B 0 2}$ & S95K000033 & 2.995 .02 & $n / a$ & $n / a$ & $n / 0$ & $n / a$ & 35.5 & no & $<3.05 E-05$ & 3.221 .05 & 1.2 \\
\hline FC. $\mathbf{0 0 7}$ & 595K000034 & $4.011-04$ & $n / a$ & n/a & $\mathrm{n} / \mathrm{a}$ & $\mathrm{n} / \mathrm{a}$ & 95.5 & na & $<3.055-05$ & $3.20 E-05$ & 11.9 \\
\hline FC.B10 & S95K000035 & $1.23 E+00$ & $n / o$ & $\mathrm{~m} / \mathrm{s}$ & $\mathrm{n} / \mathrm{s}$ & $n / a$ & 95.5 & $n / a$ & $<3.055 .05$ & $3.20 E-05$ & 0.2 \\
\hline FC-B14 & S95K000047 & $2.58 E-04$ & n/a & $n / a$ & $n / a$ & n/a & 96.4 & a/a & $3.34 E-05$ & 2.67E-05 & $\mathbf{1 4 . 5}$ \\
\hline FC.815 & $\$ 955000036$ & 3.55E.01 & $\mathbf{n / a}$ & n/a & $\mathrm{n} / \mathrm{a}$ & wa & 95.5 & n/a & $<3.05 E-05$ & 3.211 .05 & 0.4 \\
\hline FC-B16 & S95K000037 & 8.44 E.01 & $\mathrm{na}$ & $n / \mathrm{s}$ & $n / 8$ & $n / a$ & 96.4 & $\mathrm{n} / \mathrm{a}$ & $3.34 E-05$ & $2.71 E-05$ & 0.2 \\
\hline FC-B17 & S95k000038 & insullic senp & $n / a$ & $n / a$ & $n / a$ & $\mathrm{na}$ & 96.4 & $a / a$ & 3.345 .05 & $1.00 E-04$ & $n / a$ \\
\hline FC-B18 & S95K000039 & ingulfic samp & $n / a$ & $n / a$ & $n / a$ & $n / a$ & 96.4 & $n / a$ & 3.34 EE-05 & $1.00 E .04$ & n/s \\
\hline FC. 919 & $595 \times 000001$ & $1.16 E+\infty 0$ & $n / a$ & $n / a$ & nva & na & 98.2 & $n / a$ & $<3.685-03$ & $5.00 E .03$ & 2.0 \\
\hline FC-B23 & $595 K 000048$ & $4.780-01$ & $\mathrm{n} / \mathrm{a}$ & $n / a$ & $n / a$ & a/a & 96.4 & $n / a$ & 3.3415.05 & $2.69 E-05$ & 0.3 \\
\hline FC.824 & S95K000025 & 3.14E-01 & $a / a$ & $n / a$ & $n / a$ & na & 93.6 & n/a & 4.575 .03 & $3.12 E-03$ & 3.8 \\
\hline FC-825 & s95:roocoos & $5.09 E-01$ & no & $n / a$ & n/a & $n / a$ & 98.2 & $N / a$ & $<3.685-03$ & $4.10 E-03$ & 2.7 \\
\hline FC. & sseroco00s & $1.00 E+00$ & $n / a$ & n/a & $n / a$ & $n / a$ & 85.5 & $a / a$ & 4.385 .03 & $3.03 E-03$ & 20 \\
\hline FC. 329 & S95K000010 & 3.145 .01 & $n / a$ & $n / a$ & $n / a$ & $n / a$ & 95.5 & $n / n$ & $4.38 E-03$ & $3.04 E-03$ & 3.8 \\
\hline FC. 830 & $595 \times 000026$ & $2.36 E+\infty 0$ & $n / a$ & $n / a$ & $n / a$ & $n / a$ & 93.6 & $n / a$ & $4.57 E-03$ & $2.91 \mathrm{E} .03$ & 1.3 \\
\hline FC.835 & $595 \times 000011$ & $1.13 E+00$ & $\mathrm{Na}$ & $n / a$ & $n / a$ & $n / a$ & 99.4 & $n / a$ & $2.865-03$ & $4.51 E-03$ & 2.0 \\
\hline FC-837 & S05K000012 & $8.46 E-01$ & $1.26 E+\infty 0$ & $1.19 E+\infty$ & $1.13 E+00$ & 29.1 & 95.5 & 122.3 & $4.38 E-03$ & 2.98E-03 & 2.1 \\
\hline$F C .838$ & 5956000027 & $3.10 E-01$ & $n / a$ & $n / a$ & $n / a$ & $n / 4$ & 93.6 & n/a & 4.57E.03 & 2.97103 & 3.8 \\
\hline FC-B39 & $595 \times 000013$ & $7.96 E-01$ & 7.605 .01 & $7.68 E-01$ & $7.75 \mathrm{E}-01$ & 4.9 & 99.4 & 97.4 & 2.865 .03 & $4.63 \mathrm{E}-03$ & 2.4 \\
\hline FC. $B$ AO & $595 \times 000023$ & $1.72 E-01$ & $\mathrm{n} / \mathrm{a}$ & $n / a$ & $\mathrm{n} / \mathrm{e}$ & $\mathrm{n} / \mathrm{a}$ & 94.6 & $n / s$ & $7.03 E .03$ & 2.69E.03 & 5.1 \\
\hline FC-BA1 & $595 \times 000014$ & $1.08 E+00$ & $n / 0$ & $\mathrm{n} / \mathrm{a}$ & $n / a$ & $n / a$ & 99.4 & $n / a$ & $2.86 \mathrm{E}-03$ & 4.50E-03 & 2.0 \\
\hline FC.P42 & $595 \times 000015$ & $1.08 E+\infty$ & $1.10 E+\infty$ & $1.06 E+00$ & $1.08 E+00$ & 3.7 & 94.6 & 93.0 & $7.03 E-03$ & $2.97 E-03$ & 2.0 \\
\hline FC.B43 & 595x000003 & $1.51 E+0$ & $n / a$ & $n / a$ & $n / a$ & $n / 3$ & 98.2 & $n / a$ & $<3.68 E-03$ & 5.00E-03 & 1.7 \\
\hline FC. -344 & S95K000006 & $5.321-01$ & $n / s$ & $\mathrm{n} / \mathrm{a}$ & $n / a$ & $n / a$ & 98.2 & $n / a$ & $<3.68 E-03$ & $5.10 E-03$ & 3.0 \\
\hline FC-845 & $595 \times 000024$ & $4.22 E-01$ & $n / a$ & $n / a$ & $n / a$ & $n / a$ & 94.6 & $\mathrm{n} / \mathrm{a}$ & 7.03E-03 & 2.94E.03 & 3.2 \\
\hline
\end{tabular}




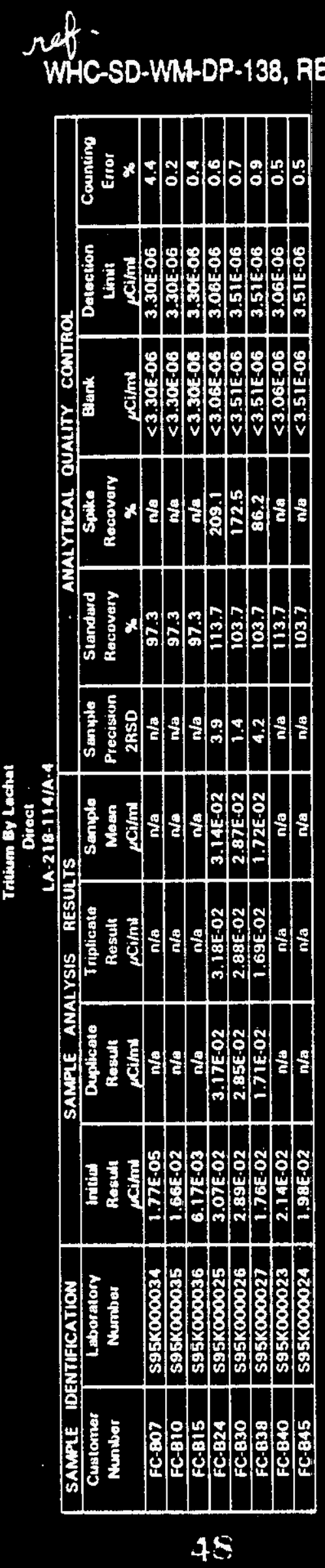




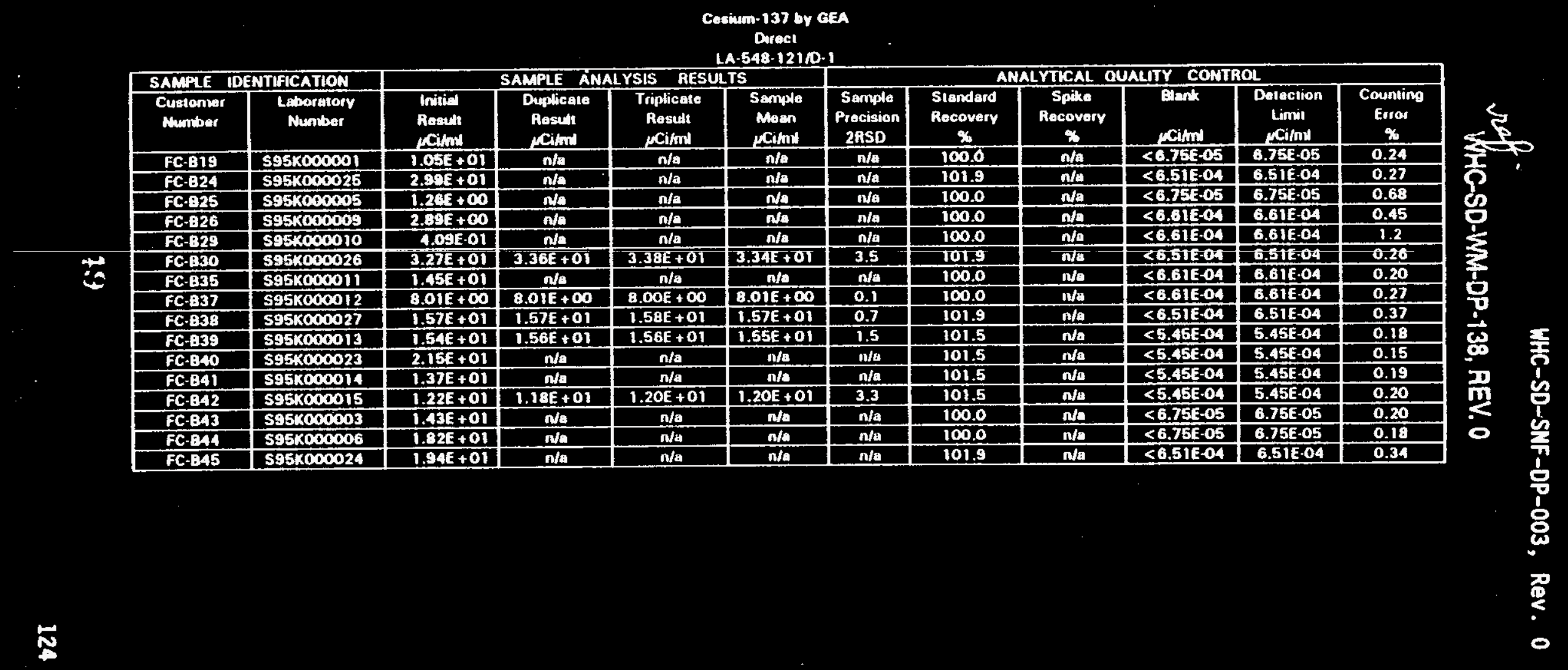




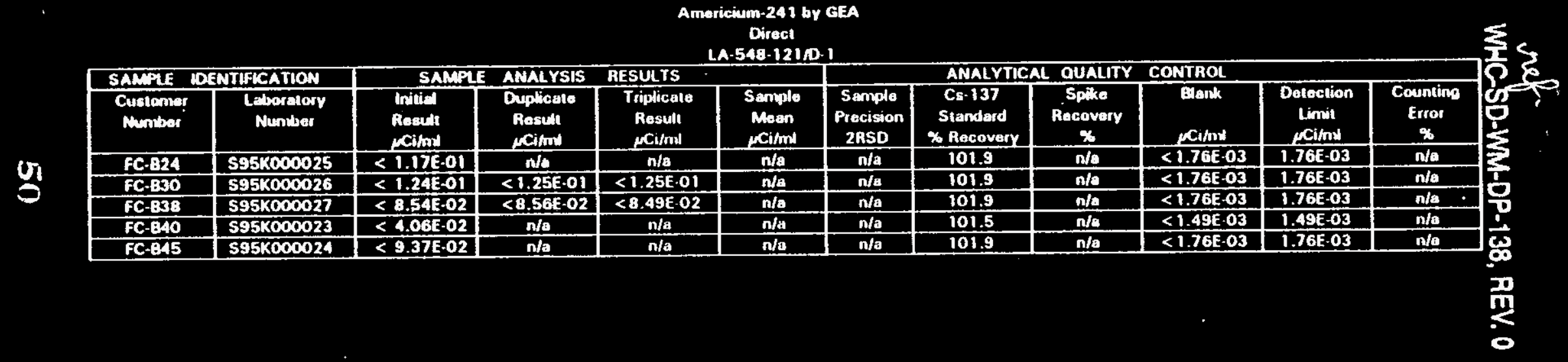

- 岕. 


\begin{tabular}{|c|c|c|c|c|c|c|c|c|c|c|c|}
\hline \multicolumn{12}{|c|}{$\begin{array}{l}\text { Am-241 by Exirzetion } \\
\text { Acid Dicestion } \\
\text { LA-953-103/A-3 }\end{array}$} \\
\hline SAMPLE II & NTIFICATION & \multirow[b]{2}{*}{$\begin{array}{l}\text { Initiad } \\
\text { Result } \\
\text { pusinert }\end{array}$} & \multirow{3}{*}{$\begin{array}{l}\text { Ampite ANA } \\
\text { Duplicale } \\
\text { Result } \\
\text { pCitoul }\end{array}$} & \multirow{2}{*}{$\begin{array}{l}\text { SIS RESU } \\
\text { Tripalicale } \\
\text { Peșult } \\
\text { pcimul }\end{array}$} & \multirow{2}{*}{$\begin{array}{l}\text { Sample } \\
\text { Moan } \\
\text { MCiml }\end{array}$} & \multirow[b]{2}{*}{$\begin{array}{l}\text { Samplo } \\
\text { Precision } \\
\text { 2RSD }\end{array}$} & \multirow[b]{2}{*}{$\begin{array}{l}\text { Sianderd } \\
\text { Recovery } \\
\%\end{array}$} & \multirow[b]{2}{*}{$\begin{array}{c}\text { Spite } \\
\text { Rocovery } \\
\%\end{array}$} & AIT cont & & \\
\hline $\begin{array}{l}\text { Cuslomer } \\
\text { Mentiber }\end{array}$ & $\begin{array}{l}\text { Loboratory } \\
\text { Crenter }\end{array}$ & & & & & & & & $\begin{array}{l}\text { Preparation } \\
\text { Blank } \\
\text { pcileml }\end{array}$ & $\begin{array}{c}\text { Dotection } \\
\text { Linnit } \\
\text { pCimul }\end{array}$ & $\begin{array}{c}\text { Counting } \\
\text { Eroot } \\
\% \\
\end{array}$ \\
\hline FC. 007 & $595 \times 000041$ & $\frac{r \text { conit }}{<2.74 E-03}$ & & $\mathrm{n} / \mathrm{a}$ & $n / a$ & $\mathrm{n} / \mathrm{a}$ & 86.6 & $n$ & $<3.51 E-03$ & $2.74 \mathrm{E} .03$ & 4.2 \\
\hline FC.B10 & $\$ 95 K 000042$ & $<2.416 .03$ & $\mathrm{n} / \mathrm{a}$ & $n / a$ & n/a & $\mathrm{ala}$ & 86.6 & n/a & $\angle 3.516-03$ & $2.41 E-03$ & 100.0 \\
\hline FC-B15 & $595 \times 000043$ & $<2.41 E-03$ & $\mathrm{n} / \mathrm{a}$ & n/a & na & $\mathrm{n} / \mathrm{a}$ & 86.6 & ala & $<3.51 \mid 03$ & $\frac{2.41 E .03}{3.908 .03}$ & $\frac{100.0}{100.0}$ \\
\hline$\frac{F C-824}{F C-830}$ & & $\frac{<3.90 \mathrm{k}-03}{<.35 E-03}$ & $<6.83 \mathrm{E}-03$ & $<2.83 E-03$ & $\mathrm{na}$ & $\mathrm{na}$ & $\begin{array}{l}96.1 \\
91.5\end{array}$ & $\frac{94.0}{82.0}$ & $\frac{<1.04 \text { E. } 02}{<5.41 E-03}$ & $\frac{3.50 E-03}{3.35 E-03}$ & $\frac{100.0}{100.0}$ \\
\hline$\frac{F C-830}{F C-B 38}$ & $\frac{595 K 000031}{595 K 000032}$ & $\begin{array}{l}<3.35 E-03 \\
<2.55 E-02\end{array}$ & $\begin{array}{l}<9.54 E \cdot 03 \\
<3.06 E-03\end{array}$ & $\begin{array}{c}<9.45 E-03 \\
<6.18 E-03\end{array}$ & $\frac{n / a}{n / a}$ & $\frac{n / a}{n / a}$ & $\frac{91.5}{93.2}$ & $\frac{82.0}{94.3}$ & $\frac{<0.418-03}{<9.64 E-03}$ & $2.55 E-02$ & 100.0 \\
\hline $\mathrm{FC}-\mathrm{B} 40$ & $595 \times 000028$ & $\frac{<2.555-02}{<2.44 E .03}$ & $\frac{<3.06 E^{-03}}{n / a}$ & $a / a$ & no & $\frac{m a}{n a}$ & 87.3 & n/a & $<2.411 .03$ & $2.44 E-03$ & 100.0 \\
\hline FC. 845 & $595 K 000029$ & $<2.526 .03$ & $n / a$ & $n / a$ & $\mathrm{n} / \mathrm{a}$ & $n / a$ & 87.3 & n/a & $<2.41 E-03$ & $2.52 E .03$ & 100.0 \\
\hline
\end{tabular}

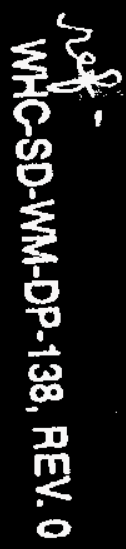




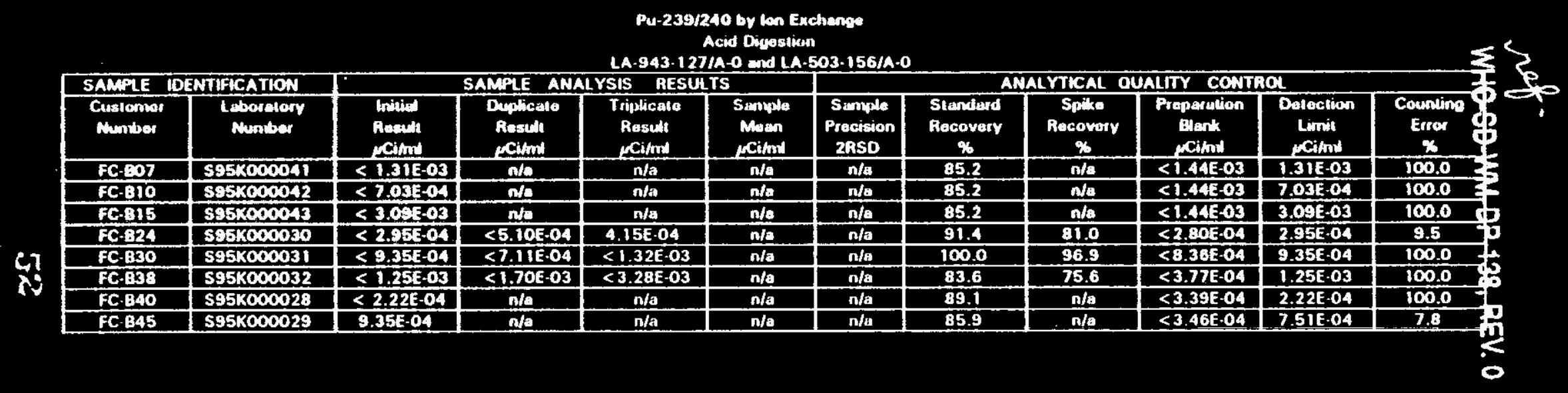


Pu-238 by lon Exchunde

Acid Digastion

LA.943.127/A.0 end LA.503.156/A-0 ANALYTICAL OUALITY CONHAOL

\begin{tabular}{|c|c|c|c|c|c|c|c|c|c|c|c|}
\hline \multicolumn{2}{|c|}{ SAMPLE DERTHFCATLOW } & \multicolumn{4}{|c|}{ SAMPLE ANALYSIS RESULTS } & \multicolumn{6}{|c|}{ ANALYTICAL OUALITY CONIROL } \\
\hline Customer & $\begin{array}{l}\text { Leboretory } \\
\text { Munces }\end{array}$ & $\begin{array}{l}\text { Initind } \\
\text { Resevh } \\
\text { ccitms }\end{array}$ & $\begin{array}{l}\text { Deplicale } \\
\text { Recult } \\
\text { pcibl }\end{array}$ & $\begin{array}{l}\text { Triplicale } \\
\text { Result } \\
\text { rCilnd }\end{array}$ & $\begin{array}{l}\text { Sanple } \\
\text { Moen } \\
\text { Cimy }\end{array}$ & $\begin{array}{l}\text { Sampla } \\
\text { Procision } \\
\text { 2ASD }\end{array}$ & $\begin{array}{c}\text { Pu.239 } \\
\text { Standad } \\
\times \text { Plocovedy }\end{array}$ & $\begin{array}{c}\text { Spike } \\
\text { Recovery } \\
\mathbf{X}\end{array}$ & $\begin{array}{l}\text { Prepartion } \\
\text { Blent } \\
\text { cilm! }\end{array}$ & $\begin{array}{l}\text { Dafoction } \\
\text { Limit } \\
\text { rcimil }\end{array}$ & $\begin{array}{c}\text { Counting } \\
\text { Error } \\
\mathbf{z}\end{array}$ \\
\hline FCED & S95K000041 & $<1.31 E-03$ & No & $n / a$ & $\sqrt{a}$ & $n \mathbf{a}$ & 85.2 & $n / 0$ & $<1.44 E .03$ & 1.311 .03 & 1000 \\
\hline FC-810 & seskcosen2 & $<7.03 E-04$ & ne & $n / a$ & n/a & nto & 85.2 & no & $<1.44 E-03$ & $7.03 E \cdot 04$ & 100.0 \\
\hline FC.815 & sstaraco43 & $<3.005-03$ & no & n/a & $n / a$ & n/a & 85.2 & nh & $<1.44 E-03$ & $3.09 E-03$ & 100.0 \\
\hline FC-824 & S95K000030 & $<2.955-04$ & $<5.10 .04$ & $<3.05 E-04$ & $n / a$ & $\mathrm{n} / \mathrm{a}$ & 91.4 & $n / a$ & $<2.80 E-04$ & $2.95 E-04$ & 1000 \\
\hline FC. $-\mathrm{B30}$ & $595 \times 000031$ & $<9.355-04$ & $<7.11 E-04$ & $<1.32 E .03$ & n/a & $n / \mathrm{s}$ & 100.0 & $n / a$ & $<8.36 .6 .04$ & 9.35E.04 & 100.0 \\
\hline FC-B38 & $595 \times 000032$ & $<1.25 E-03$ & $<1.70 \mathrm{E}-03$ & $<3.28 E-03$ & $n / 0$ & $n / a$ & 83.6 & $\mathrm{n} / \mathrm{a}$ & $<3.77 \mathrm{E}-04$ & $1.25 E-03$ & 11.3 \\
\hline FC- 040 & SS5K000023 & $<2.225 .04$ & $\mathrm{n} / \mathrm{a}$ & $\mathrm{n} / \mathrm{a}$ & $n / a$ & $n / a$ & 89.1 & $\mathrm{n} / \mathrm{a}$ & $<3.39 E .04$ & $2.22 E-04$ & 100.0 \\
\hline$F C \cdot B A 5$ & $595 k 000029$ & $<7.51 E .04$ & $n / \mathrm{s}$ & $n / a$ & n/a & $n / a$ & 85.9 & $n / a$ & $<3.46 E-04$ & 7.51E-04 & 11.5 \\
\hline
\end{tabular}


DISTRIBUTION SHEET

\begin{tabular}{|c|c|c|c|c|c|c|}
\hline \multirow{2}{*}{$\begin{array}{l}\text { To } \\
\text { Distribution }\end{array}$} & \multirow{2}{*}{$\begin{array}{l}\text { From } \\
\text { Spent } \\
\text { 8in710 }\end{array}$} & \multirow{2}{*}{\multicolumn{3}{|c|}{ Nuclear Fuel Evaluations }} & \multicolumn{2}{|l|}{ Page 2 of 2} \\
\hline & & & & & \multicolumn{2}{|c|}{ Date $12 / 20 / 95$} \\
\hline \multirow[t]{2}{*}{ Project Titte/Work Order } & \multirow[t]{2}{*}{$\cdot$} & & & & \multicolumn{2}{|c|}{ EDT No. 610186} \\
\hline & & & & & \multicolumn{2}{|l|}{ ECN No. } \\
\hline Name & & MSIN & $\begin{array}{l}\text { Text } \\
\text { With All } \\
\text { Attach. }\end{array}$ & Text Onl & $\begin{array}{l}\text { Attach./ } \\
\text { Appendix } \\
\text { Only }\end{array}$ & $\begin{array}{l}\text { EDT/ECN } \\
\text { Only }\end{array}$ \\
\hline
\end{tabular}

\section{Mextinohoure Hanford Commany Continued}

W. C. Mills

C. R. Miska

R. P. Ouberg

A. L. Pitner

R. D. Prescott

G. W. Reddick Jr.

E. F. Riedel

J. P. Schmidt

P. K. Shen

D. L. Sherrel1

$\times 3-79$

R3-86

R3-85

L5-01

L5-0I

H5- 49

S3-90

$\times 3-78$

R3-87

R3-86

D. W. Siddoway

$\times 3-71$

D. W. Smith

D. J. Trimble

D. J. Watson

K. L. Noodard

Central files

(2)

$\times 3-80$

L5-01

X3-79

L5-01

A3-88

$x$
$x$
$x$
$x$
$x$
$x$
$x$
$x$
$x$
$x$
$x$
$x$
$x$
$x$
$x$
$x$




\begin{tabular}{|c|c|c|c|c|c|}
\hline \multicolumn{6}{|c|}{ DISTRIDUTION SHEET } \\
\hline To & \multirow{2}{*}{\multicolumn{3}{|c|}{$\begin{array}{l}\text { From } \\
\text { Spent Nuclear Fuel Evaluations } \\
\text { Bin710 }\end{array}$}} & \multicolumn{2}{|l|}{ Page 1 of 2} \\
\hline Distribution & & & & \multicolumn{2}{|c|}{ Date $12 / 20 / 95$} \\
\hline \multirow[t]{2}{*}{ Project Title/Work Order } & & & & \multicolumn{2}{|c|}{ EDT No. 610186} \\
\hline & & & & \multicolumn{2}{|c|}{ ECN No. } \\
\hline Name & MSIN & $\begin{array}{l}\text { Text } \\
\text { With All } \\
\text { Attach. }\end{array}$ & Text On & $\begin{array}{l}\text { Attach./ } \\
\text { Appendix. } \\
\text { Only }\end{array}$ & $\begin{array}{c}\text { EDT/ECN } \\
\text { Only }\end{array}$ \\
\hline
\end{tabular}

U.S. Damentment of Enorav.

Rich and Fiald office

D. C. Bryson

G. D. Trenchard

S7-41

$57-41$

E. D. Sellers

$57-41$

J. Shuen

$57-41$

$x$
$X$
$X$
$X$

Mac Technical Sarvices Comnany

G. Baston

R. P. Denise

J. C. Devine

R3-82

R3-82

R3-82

$x$
$x$
$x$

Pacific Northwest Laboratory

J. Abrefah

A. B. Johnson

S. C. Marschman

P7-14

K8-34

P7-18

P. A. Scott

T. A. Thornton

R3-87

P7-18

Mestinghouse Hanford Company

C. J. Alderman

R. B. Baker

D. W. Bergmann

K. H. Bergsman

A. E. Bridges

L. D. Bruggeman

S. P. Burke

S. A. Chastain

K. R. Conn

R. G. Cowan

D. R. Duncan

J. R. Frederickson

J. C. Fulton

R. G. Gant

E. W. Gerber

L. H. Goldman

M. A. Green

R. A. Harris

S. A. Hecht

A. T. Kee

M. J. Langevin

R3-48

L5-01

R3-86

R3-86

L5- 01

L5- 01

X3-74

L5-01

$\times 3-79$

R3-86

R3-86

R3-86

R3-11

X3-79

R3-86

R3-86

$\times 3-67$

L5-01

L5-01

R3-86

$\times 3-74$

L. A. Lawrence

L5-01

L5-01

B. J. Makenas

$\mathrm{R3}-48$

$x$
$x$
$x$
$x$
$x$

L. M. Mellethy 